\title{
Optimal FRP reinforcement of masonry walls out-of-plane loaded: a combined homogenization-topology optimization approach complying with masonry strength domain
}

\author{
by \\ Matteo BRUGGI ${ }^{(1)} \&$ Gabriele MILANI*(2) \\ (1) Department of Civil and Environmental Engineering DICA, Politecnico di Milano, Piazza \\ Leonardo da Vinci 32, 20133, Milan, Italy \\ (2) Department of Architecture, Built Environment \& Construction Engineering ABC, \\ Politecnico di Milano, Piazza Leonardo da Vinci 32, 20133, Milan, Italy \\ * Corresponding author.E-mail: gabriele.milani@polimi.it \\ Phone: +39022399 4290 Fax: +390223994220
}

\begin{abstract}
A novel approach for the rational arrangement of fiber reinforcements on masonry structures based on topology optimization is presented. Following previous experiences on the automatic achievement of strut-and-tie models in reinforced concrete structures, the minimization of the strain energy can be implemented to derive optimal layouts of reinforcement for any structural element. To cope with the brickwork limited strength, the optimal problem can be conveniently reformulated as the minimization of the amount of reinforcement that is required to keep tensile stresses in any masonry element below a prescribed threshold. The out-of-plane macroscopic elastic properties and strength domain of brickwork are derived through an original homogenization approach, which relies upon the discretization of $1 / 4$ of any unit cell by six constant moment elements. Thanks to the limited number of variables involved, fast evaluations of masonry macroscopic strength domain can be obtained. This criterion is implemented into the multi-constrained discrete formulation of the topology optimization algorithm, to locally control the internal actions field over the design domain. Topology optimization is then applied to the investigation of the optimal reinforcement of plain and windowed panels, comparing the conventional energy-based method and the proposed stress-based approach.
\end{abstract}

Keywords: masonry, reinforcement, out-of-plane loads, homogenization, topology optimization, minimum compliance, local stress constraints 


\section{Introduction}

Masonry out-of-plane failures are very common in presence of deformable floors [1] and occur always at very low levels of the horizontal actions [2]-[6], especially for historical buildings with poor mechanical properties of the joints and for slender perimeter walls.

From both experimental experience and post-earthquake surveys, it can be stated that out-of-plane damages regard slender walls, for which it is acceptable to assume that masonry behaves as a thin Kirchhoff-Love plate [4], so that, at the macro-scale, almost every out-of-plane damage mechanism can be described as a combination of three fundamental meso-scale mechanisms: vertical bending, horizontal bending, and torsion.

Another important issue that influences both strength and ductility is the static vertical compression, due to the gravity loads. As well known, indeed, gravity plays a fundamental role in the definition of the moment-curvature non-linear relationship, at a sectional level, in conjunction with the texture geometry of the masonry.

Orthotropy is another key feature, and it is significantly related to masonry texture, especially for horizontal bending, i.e. with rotation along a vertical axis [4]-[7]. Texture produces perceivable effects that tend to become more evident with the progressive degradation of the material. Mainly, the different topology of the continuous horizontal mortar joints with respect to the vertical ones, interrupted by the blocks, implies that mortar shear response tends to play a significant role under horizontal bending, while it is substantially not influent in the vertical bending response.

In this context, it appears paramount for design, either a refurbishment or upgrading of existing masonry panels, especially where horizontal actions are expected to occur (i.e. in seismic zone). To increase the out-of-plane bearing capacity of masonry walls, it is possible to utilize conventional retrofitting techniques, such as external reinforcement with steel plates, surface concrete coating and welded mesh. Unfortunately they have proven to be impractical, time expensive and add 
considerable mass to the structure. In general, this latter feature is generally considered a critical drawback in seismic zone, because it increases inertia forces induced by earthquakes.

All things considered, the use of Fiber Reinforced Polymers (FRPs) to strengthen or retrofit existing masonry [8]-[15] may have particular appeal, including this kind of structural reinforcement flexibility, reversibility, and limited increase in structural weight.

So far, the layout of the reinforcing strips has been basically driven by the intuition, owing to the simplicity of the loading conditions in the case of laboratory samples, or by the intent of healing existing cracks in the case of real structures.

An alternative, which however is still heuristic, is to perform Finite Element computations on the reinforced structures and try to dispose strips in such a way to close active cracks forming the failure mechanism [15]. While this latter approach is quite straightforward, it has the rather relevant disadvantage of requiring a superabundant reinforcement, because the reinforcement is placed perpendicularly to all yield lines forming the failure mechanism. Conversely, a desired increase of the load bearing capacity could be obtained simply with the inhibition of the first cracks forming under the application of the loads.

Generally speaking, in presence of complex geometries, constraints and loading conditions, more sophisticated approaches with topology optimization base should be used. An attempt in this end was made by Krevaikas and Triantafillou [16], who identified the optimal layout of FRP strips to be placed on in-plane loaded masonry walls. The approach was on a rational basis, by preliminarily defining a grid through which the optimal reinforcing array has to pass.

Recently, an innovative methodology based on the combined utilization of homogenization concepts and topology optimization was proposed both for in-plane loaded masonry structures in [17] with stress-constraints imposed on the masonry layer, and for out-of-plane loaded masonry in [18] in the framework of a standard energy based procedure. Both approaches proved robustness and promising flexibility, without requiring any a-priori assumption on the optimal reinforcing layout. In the present work, a topology optimization approach with stress constraints is extended to 
the case of masonry walls out-of-plane loaded. At this aim, the simple homogenization model proposed in the elastic range in [18] to quickly estimate flexural masonry homogenized stiffness, is extended to the limit analysis case (both bricks and mortar are assumed as rigid-perfectly plastic materials with infinite ductility) for a fast and reliable evaluation of the macroscopic masonry outof-plane strength domain.

The model relies into the subdivision of the elementary cell into 24 constant moment triangular elements. Imposing equilibrium on interfaces and anti-periodicity conditions at the boundary of the elementary cell, the determination of both elastic moduli and failure surfaces involves a very limited number of unknowns. The elastic problem is solved on the unit cell by means of the constrained minimization of the complementary energy, whereas the determination of the homogenized strength domain is handled within the classic lower bound theorem of limit analysis and thus solved with the recursive solution of linear programming problems.

A wide literature exists about the automatic achievement of strut-and-tie models in reinforced concrete structures via topology optimization, see e.g. [19], suggesting the adoption of the strain energy as objective function to be minimized with the aim of providing an optimal reinforcement for any structural element. However, such procedure does not account for the limited masonry strength in tension. In this paper, this crucial issue is tackled, conveniently reformulating the optimal problem as the minimization of the amount of reinforcement that is needed to keep tensile stresses in any masonry element below a prescribed threshold.

Once the homogenized behavior of the wall in the elastic range and at failure is known, a topology optimization problem with constraints on the moments acting on the masonry layer is therefore proposed to estimate the optimal layout of the external FRP reinforcement that complies with the assigned out-of-plane load, without violating in any point the admissible internal actions state for masonry elements. In particular, the present contribution investigates numerical features and optimal layouts achieved by the proposed stress-based approach, providing a comparison with the conventional energy-based approach as well. 
The paper is organized as follows: in Section 2, the out-of-plane homogenization problem is briefly recalled, whereas is Sections 3 and 4, respectively the elastic and limit analysis homogenization models for masonry loaded out-of-plane are presented. The approach is tested on both the linear elastic and limit analysis case, comparing results obtained with alternative approaches available in the literature and FE computations when dealing with the elastic case. The topology optimization problem that allows for the optimal reinforcement of a masonry panel in bending is formulated in Section 5. Section 6 is finally dedicated to the detailed analysis of three structural problems, where the optimal reinforcing layouts for a solid and two windowed panel are derived.

\section{Masonry homogenization, simple out-of-plane model in the linear elastic and limit analysis case}

Let us consider an out-of-plane loaded masonry wall, relying into a regular pattern of units, separated by bed and head mortar joints. Owing to the material periodicity, a single unit cell $(Y)$ can be used as Representative Volume Element (RVE) for the heterogeneous medium, that is, the smallest volume element containing all the information necessary to completely describe the macroscopic behavior of the entire wall (see Figure 1). If a running bond (or a header bond) pattern is considered, as shown in Figure 1, it is expedient to adopt a unit cell of rectangular shape.

Homogenization is a convenient strategy to analyze masonry structures, both in the linear and in the non-linear range, since the mechanical properties of the constituent materials (bricks and mortar) are accounted for only at the cell level, and large scale FE computations at the macro-scale can be performed without the need of meshing joints and bricks separately.

Homogenization has long been used for the analysis of in-plane loaded masonry structures [20][27], and has recently been extended to masonry walls subjected to out-of-plane loads (see e.g. [5][15][28]-[31] etc.).

According to homogenization theory for heterogeneous bodies in bending [29], averaged quantities representing the macroscopic curvature and moment tensors (denoted by $\chi$ and $\mathbf{M}$, respectively) are defined as: 
$\chi=\frac{1}{V} \int_{Y} \frac{\boldsymbol{\varepsilon}(\mathbf{u})}{Z} d Y \quad \mathbf{M}=\frac{1}{A} \int_{Y} z \boldsymbol{\sigma} d Y$

where $Y$ denotes the elementary cell, $V$ its volume, $A$ the area of the cell in the $x$-y plane, $\boldsymbol{\varepsilon}(\mathbf{u})$ and $\boldsymbol{\sigma}$ the local (microscopic) strains and stresses, respectively and $\mathbf{u}$ the displacement field inside the RVE.

The local stress $\boldsymbol{\sigma}$ and displacement $\mathbf{u}$ fields must fulfill suitable periodicity conditions that read:

$\left\{\begin{array}{l}\mathbf{u}=z \chi \mathbf{v}+\mathbf{u}^{\text {per }} \text { in } Y \\ \mathbf{M n} \text { anti-periodic on } \partial Y\end{array}\right.$

where $\mathbf{u}^{\text {per }}$ is the periodic part of the displacement field, $\mathbf{v}$ is any point in the RVE at $z=0$ in the local reference frame, $\partial Y$ is the boundary of the RVE and $\mathbf{n}$ is the unit outward normal vector to $\partial Y$ (see Figure 1).

To analyze the macroscopic elastic behaviour of a masonry wall in bending, the simplest hypothesis that can be done is assuming both constituent materials to be linearly elastic, with joints reduced to interfaces of vanishing thickness. It this framework, units are discretized by means of a coarse mesh consisting of constant moment triangles (CMT), independently formulated by Hellan [32] and Herrmann [33], as sketched in Figure 1. This type of triangular element has been preferred to other more accurate elements, such as those proposed by Krenk et al. [34] and Krabbenhoft and Damkilde [35], due to its simplicity and to the low number of unknowns involved in the optimization procedure (see Sec. 4).

A constant moment field is assumed within each element, so that three moment unknowns per element are introduced. These unknowns are the bending moments (per unit length) about the horizontal and vertical axis, and the twisting moment $\left(M_{x x}, M_{y y}, M_{x y}\right)$, see Figure 1.

The choice of meshing 1/4 of the brick through at least 3 triangular elements is due to the need of reproducing the presence of twisting in the bed joints (element 2 in Figure 1) under horizontal bending. In this way, and with the coarse discretization adopted, 1/4 of the RVE is meshed through 
6 triangles, indicated in Figure 1 by $1,2,3,7,8,9$. The generalization of the symbols to the whole cell is straightforward.

From here onwards, the superscript ${ }^{(n)}$ will indicate any moment component belonging to the $n$-th element. Accordingly, assuming the wall to undergo thin-plate conditions, the moment tensor in the $n$-th CMT element, $\mathbf{M}^{(n)}$, is characterized by the three non-vanishing components $M_{x x}^{(n)}$ (horizontal bending), $M_{y y}^{(n)}$ (vertical bending) and $M_{x y}^{(n)}$ (torque).

Assuming a stress-based approach, and neglecting both body forces and out-of-plane tractions, equilibrium within any element is a-priori satisfied, being the moment tensor element-wise constant $\left(\partial^{2} M_{x x}^{(n)} / \partial x^{2}+\partial^{2} M_{x y}^{(n)} / \partial y^{2}=0\right.$ and $\left.\partial^{2} M_{x y}^{(n)} / \partial x^{2}+\partial^{2} M_{y y}^{(n)} / \partial y^{2}=0\right)$. On the contrary, two equality constraints involving bending and twisting moments along the interface between adjoining triangular elements have to be prescribed at any internal interface. For instance, the moment vector must be continuous across the interface between elements 1 and 2 . It can be easily shown that the moment components in elements (1) and (2) are linked by the following two equations:

$$
\begin{aligned}
& M_{x x}^{(2)}=M_{x x}^{(1)}+\zeta\left(M_{x y}^{(1)}-M_{x y}^{(2)}\right) \\
& M_{y y}^{(2)}=M_{y y}^{(1)}+\zeta^{-1}\left(M_{x y}^{(1)}-M_{x y}^{(2)}\right)
\end{aligned}
$$

having denoted by $\zeta$ the ratio of the semi-length to the height of the brick $(\zeta=b / 2 a)$. Similar equations must be written at all the remaining interfaces, which are globally 28. A total of 56 equilibrium equations at the interfaces is obtained, whereas 72 are the unknown internal actions components (three for each triangular element).

Anti-periodicity constraints for the moment vector are prescribed on the couples of triangles 1-6, 712, 13-18, 19-24, 1-19, 3-21, 4-22, 6-24, leading to additional 16 equalities. For instance, referring to couple 1-6, moment anti-periodicity amounts at setting:

$$
\begin{aligned}
& M_{x x}^{(1)}=M_{x x}^{(6)} \\
& M_{x y}^{(1)}=M_{x y}^{(6)} .
\end{aligned}
$$


Not all the equations are, however, linearly independent. In particular, it can be shown that the corner elements 1, 6, 19 and 24 provide 4 linearly dependent equations for the twisting moment.

\section{Homogenized masonry elastic moduli}

The homogenized flexural rigidities can be obtained with the procedure envisaged in [21][18], where the reader is referred for details, minimizing the complementary energy in the unit cell - see a similar procedure for the in-plane case in [21]. In this case, the complementary energy is given by the following quadratic form:

$$
\begin{aligned}
\Pi^{*} & =\frac{1}{2} \sum_{i=1}^{N^{t r}} A_{t r}^{(i)} \frac{12\left(1-v_{b}^{2}\right)}{E_{b} t^{3}}\left[M_{x x}^{(i)^{2}}-2 v_{b} M_{x x}^{(i)} M_{y y}^{(i)}+M_{y y}^{(i)^{2}}+2 \frac{M_{x y}^{(i)}{ }^{2}}{\left(1-v_{b}\right)}\right]+\frac{1}{2} \sum_{i=1}^{N^{I}} A_{I}^{(i)} \frac{12}{t^{3}}\left[\frac{M_{n n}^{(i)^{2}}}{E_{m}}+\frac{M_{n t}^{(i)^{2}}}{G_{m}}\right] \\
& -t M_{i j} \chi_{i j},
\end{aligned}
$$

where $N^{t r}$ is the number of triangular elements, of area $A_{t r}^{(i)}, N^{I}$ is the total number of mortar interfaces, $A_{I}^{(i)}$ is the area of the i-th mortar interface, $\chi_{i j}$ are prescribed macroscopic curvature components conjugated to the three macroscopic moments $M_{i j}$. Summation over $i$ and $j$ is implied ( $i$, $j=x$ or $y)$,

The determination of the plate elastic moduli can be obtained by a constrained minimization of the complementary energy, which is a quadratic form in the 72 independent moment components in the elements and the three macroscopic moments.

In order to test the reliability of the procedure proposed, a technically meaningful example is analyzed in detail, consisting of a masonry wall with bricks of dimensions $215 \times 65 \times 102.5 \mathrm{~mm}^{3}$ (length $\times$ height $\times$ thickness) disposed in running bond, and mortar joints $5 \mathrm{~mm}$ thick.

Here it is worth noting that such example refers to some windowed panels tested out-of-plane up to collapse by Chong and co-workers [36][37] and that the orthotropic stiffness matrix here derived will be used within the topology optimization problem discussed next. 
Thanks to the very limited number of optimization variables involved, a standard large scale quadratic programming routine is utilized to solve the elastic problem on the unit cell, eq. (5), varying the ratio of the elastic moduli of brick and mortar $\left(E_{b} / E_{m}\right)$ over a wide range.

The mechanical properties of the constituent materials are summarized in Table I; the mortar joint elastic modulus is progressively decreased from the value given in Table I down to 1/35 of the brick elastic modulus.

Results of the simulations are summarized in Figure 2, where a comparison with results obtained utilizing a standard 3D FE discretization of the elementary cell is reported. The FE procedure adopted is an out-of-plane homogenization, firstly proposed for this kind of problems in [29]. The linear elastic homogenization problem, as well as the periodicity conditions to be applied within a commercial FE code to get the homogenized elastic coefficients, are discussed in detail in [24][29][30]. The FE discretization adopted is depicted in Figure 3, it is sufficiently refined and authors experienced very little differences on homogenized elastic moduli with a further reduction of the mesh size.

$D_{i j h k}$ moduli obtained using Lourenço [38] and Zucchini \& Lourenço [26] models are also reported for the sake of completeness. According to the in-plane macroscopic stiffness coefficients $A_{i j h k}$ predicted by these models, the flexural rigidities $D_{i j h k}$ are computed as $A_{i j h k} t^{3} / 12$, $t$ being the wall thickness.

The procedure proposed by Zucchini \& Lourenço [26] belongs to the wide family of in-plane models (see also [22][23]). This procedure has proved to be relatively reliable and allows the homogenized elastic moduli to be obtained solving a set of simplified compatibility and equilibrium equations deduced from the behavior of a unit cell discretized by means of 3D finite elements. The disadvantage is that the procedure holds for membrane loads, and may lead to incorrect predictions of the flexural rigidity about an horizontal axis, which is significantly affected by twisting of the mortar joints, especially if blocks are rigid or the brick-to-mortar Young's modulus ratio is large. 
The procedure proposed by Lourenço [38] consists in homogenizing a masonry pillar constituted by two half bricks (of height $h$ ) and a joint (of thickness $e$ ). Accordingly, it can be regarded as a variation of the procedure by Pande et al. [22] applied to the evaluation of the vertical membrane stiffness exclusively. For such a structural system, the flexural rigidity about a horizontal axis can be deduced in closed form and is provided by the following formula:

$$
D_{y y y y}=\frac{t^{3}}{12} \frac{h+e}{\left(1-v_{b}^{2}\right) \frac{h}{E_{b}}+\left(1-v_{m}^{2}\right) \frac{e}{E_{m}}}
$$

The error estimation depicted in Figure 2-b shows that in most cases the present approach fits well the results obtained with the previously presented simplified approaches, even in presence of quite weak mortar joints. There is a significant deviation from Zucchini \& Lourenço's model only in terms of $D_{x x x x}$ as $E_{b} / E_{m}>20$ for the reason discussed above.

\section{Homogenized strength domain}

When dealing with rigid-perfectly plastic materials, a particularly simple lower bound problem of limit analysis is obtained. With the same rough discretization adopted within the linear elastic case, unknowns involved into the limit analysis problem are internal variables (bending moment and torque of each element) and the external load multiplier. Considering all the REV, total unknowns are therefore $3 \times 24+1=73$, including also the load multiplier. Within the limit analysis problem, 68 linearly independent equations must be considered, derived from equilibrium at the edge of adjoining elements and anti-periodicity. Finally, a set of inequality constraints representing the yield conditions at the interfaces and on elements, involving bending moment and torque unknowns, fully defines the optimization problem. The objective function, in the framework of the lower bound theorem of limit analysis, is represented by the load multiplier. 
To estimate a single point of the out-of-plane homogenized yield domain in the $M_{x x}-M_{y y}-M_{x y}$ space, it is necessary to select a direction vector $\mathbf{n}_{M}=\left[\begin{array}{lll}\alpha & \beta & \gamma\end{array}\right]^{T}$ so that the point on the failure surface to be found is $\lambda \mathbf{n}_{M}$, and to solve the following non-linear programming (NLP) problem:

$\max \lambda \quad$ subject to $\left\{\begin{array}{l}\lambda \alpha=\frac{\sum_{i=1}^{24} M_{x x}^{(i)} A_{i}}{2 a b} \\ \lambda \beta=\frac{\sum_{i=1}^{24} M_{y y}^{(i)} A_{i}}{2 a b} \\ \lambda \gamma=\frac{\sum_{i=1}^{24} M_{x y}^{(i)} A_{i}}{2 a b} \\ \mathbf{A}_{e q}^{I} \mathbf{X}=\mathbf{b}_{e q}^{I} \\ \mathbf{A}_{e q}^{a p} \mathbf{X}=\mathbf{b}_{e q}^{a p} \\ f_{E}^{i}\left(M_{x x}^{(i)}, M_{y y}^{(i)}, M_{x y}^{(i)}\right) \leq 0, i=1 \ldots 24 \\ f_{I}^{i}\left(M_{n n}^{(i)}, M_{n t}^{(i)}\right) \leq 0, i=1 \ldots 32\end{array}\right.$

The symbols used in equation (7) have the following meaning:

- $\quad A_{i}$ is the area of the i-th element ( $a b / 8$ or $\left.a b / 16\right)$;

- $\quad \boldsymbol{X}$ is a $73 \times 1$ of optimization unknowns (elements moments and collapse multiplier);

- $\quad \boldsymbol{A}_{e q}^{I} \boldsymbol{X}=\boldsymbol{b}_{e q}^{I}$ is a set of linear equations collecting equilibrium constraints on all the interfaces. $\boldsymbol{A}_{e q}^{I}$ is a $56 \times 73$ matrix and $\boldsymbol{b}_{e q}^{I}$ is a $56 \times 1$ array with entries equal to zero;

- $\boldsymbol{A}_{e q}^{a p} \boldsymbol{X}=\boldsymbol{b}_{e q}^{a p}$ collects the anti-periodicity conditions and it is therefore a set of 16 equations (some of them linearly dependent). Thus $\boldsymbol{A}_{e q}^{a p}$ is a $16 \times 73$ matrix and $\boldsymbol{b}_{e q}^{a p}$ is a $16 \times 1$ array with entries equal to zero;

- $\mathbf{f}_{E}^{i}\left(M_{x x}^{(i)}, M_{y y}^{(i)}, M_{x y}^{(i)}\right) \leq \mathbf{0}$ is a set of possibly non-linear inequalities constraints representing the failure surface adopted for the $i$-th element;

- $\mathbf{f}_{I}^{i}\left(M_{n n I}^{(i)}, M_{n t I}^{(i)}\right) \leq \mathbf{0} \forall i=1, \ldots, 32$ plays the role of $f_{E}^{i}$ for the interfaces, with $M_{n n I}^{(i)}$ and $M_{n t I}^{(i)}$ indicating respectively the bending moment and torsion acting on the $i$-th interface.

When dealing with brick triangles (continuum), since Kirchhoff-Love plate elements are utilized to discretize the elementary cell, a failure surface in the $M_{x x}-M_{y y}-M_{x y}$ space is needed. Such failure surface is typically obtained subdividing the element along the thickness $t$ into several layers, say $n_{I}$, and assuming for each layer a Mohr-Coulomb failure criterion in plane stress. Within such 
assumption, the following lower bound limit analysis problem is solved to find a point belonging to the failure surface assumed for the triangular element (i.e. $\left.\mathbf{f}_{E}^{i}\left(M_{x x}^{(i)}, M_{y y}^{(i)}, M_{x y}^{(i)}\right)=\mathbf{0}\right)$ in the $M_{x x}-M_{y y}-M_{x y}$ space, with pre-assigned direction defined by the unitary vector $\mathbf{n}_{M}=\left[\begin{array}{lll}\alpha & \beta & \gamma\end{array}\right]^{T}$

$$
\max \lambda\left\{\begin{array}{c}
\lambda \alpha=\sum_{i=1}^{n_{I}} \sigma_{x x}^{(i)}\left(-\frac{t}{2}+i \frac{t}{n_{I}}-\frac{t}{2 n_{I}}\right) \frac{t}{n_{I}} \\
\lambda \gamma=\sum_{i=1}^{n_{I}} \sigma_{x y}^{(i)}\left(-\frac{t}{2}+i \frac{t}{n_{I}}-\frac{t}{2 n_{I}}\right) \frac{t}{n_{I}} \\
\lambda \beta=\sum_{i=1}^{n_{I}} \sigma_{y y}^{(i)}\left(-\frac{t}{2}+i \frac{t}{n_{I}}-\frac{t}{2 n_{I}}\right) \frac{t}{n_{I}} \\
\sum_{x x}^{n_{I}} \sigma_{x x} t \\
N_{i=1} N_{y y}=\frac{\sum_{i=1}^{n_{I}} \sigma_{y y} t}{n_{I}} \sigma_{x y} t \\
f^{i}\left(\sigma_{x x}^{(i)}, \sigma_{y y}^{(i)}, \sigma_{x y}^{(i)}\right) \leq \mathbf{0}
\end{array}\right.
$$

Where $f^{i}\left(\sigma_{x x}^{(i)}, \sigma_{y y}^{(i)}, \sigma_{x y}^{(i)}\right)=0$ is the strength domain of the i-th layer and the external membrane compressive load ( $N_{x x}, N_{y y}$ and $N_{x y}$ ) is assumed a priori known. Typically, it is reasonable to assume $N_{x x}$ and $N_{x y}$ equal to zero and $N_{y y}$ equal to masonry self-weight, see [39]. Within such a simplification, at a distance $h$ from the top of the wall, the vertical pre-compression is simply $N_{y y}=-\gamma_{M} h$, where $\gamma_{M}$ is masonry specific weight. Generally, this assumption is technically acceptable for experimental tests, since in these cases a fixed in-plane compressive load (regarded as permanent load) $N_{y y}=-N_{0}$ is applied before out-of-plane actions and kept constant until failure, whereas $N_{x x}=N_{x y}=0$.

Similar considerations may be repeated to find the out-of-plane strength domain of element-element interfaces. In this latter case, however, it must be noted that two different typologies of interfaces are present in the model, namely brick-brick common edges and mortar joints.

In order to evaluate their strength domain in the bending moment-torsion plane $M_{n n}-M_{n t}$ it is again necessary to subdivide the interface into several layers along the thickness and for each layer select a suitable failure criterion in the in-plane stress space $\sigma_{I}^{(i)}-\tau_{I}^{(i)}$ (normal stress-tangential stress). Then the following lower bound limit analysis problem is written, which allows determining a point of the failure surface in the $M_{n n}-M_{n t}$ plane, along the direction defined by the unitary vector $\mathbf{n}_{M_{I}}=\left[\begin{array}{ll}\alpha & \beta\end{array}\right]^{T}$ 


$$
\max \lambda\left\{\begin{array}{c}
\lambda \alpha=\sum_{i=1}^{n_{I}} \sigma_{I}^{(i)}\left(-\frac{t}{2}+i \frac{t}{n_{I}}-\frac{t}{2 n_{I}}\right) \frac{t}{n_{I}} \\
\lambda \beta=\sum_{i=1}^{n_{I}} \tau_{I}^{(i)}\left(-\frac{t}{2}+i \frac{t}{n_{I}}-\frac{t}{2 n_{I}}\right) \frac{t}{n_{I}} \\
N_{I \sigma}=\frac{\sum_{i=1}^{n_{I}} \sigma_{I} t}{n_{I}} \\
N_{I \tau}=\frac{\sum_{i=1}^{n_{I}} \tau_{I} t}{n_{I}} \\
f_{I}^{i}\left(\sigma_{I}^{(i)}, \tau_{I}^{(i)}\right) \leq 0
\end{array}\right.
$$

Where $f_{I}^{i}\left(\sigma_{I}^{(i)}, \tau_{I}^{(i)}\right) \leq 0$ is the in-plane failure surface of the i-th layer and $N_{I \sigma}$ and $N_{I \tau}$ are assigned membrane actions (normal and tangential component respectively) acting on the interface.

For joints, a Mohr-Coulomb failure criterion with tension cutoff and linearized cap in compression is adopted, see Figure 4 where plastic multiplier rates for each segment (under the hypothesis of associated plasticity) are also represented, with mechanical parameters defining the failure surface as follows: $f_{t}$ is the tensile strength, $c$ is the cohesion, $\Phi$ is the friction angle, $\Psi$ the shape of the linearized compressive cap and $f_{c}$ the compressive strength.

In Figure 5, the out-of-plane joint failure surface at different levels of membrane load acting perpendicularly to the interface is shown, assuming the mechanical properties as in Table II (only strength values are considered), with a thickness $t$ of the interface equal to $102.5 \mathrm{~mm}$ (which corresponds to the thickness of the walls tested by Chong and co workers [36][37] and analyzed in the following Sections). Each point of the failure surface is estimated solving the optimization problem (9).

As expected, a moderate membrane compression results to be quite beneficial for both the torsional and flexural strength of the interface, but when the compression approximates the ultimate strength $f_{c}$, a visible drop of the out-of-plane strength is observed.

For bricks (triangular elements), a Mohr-Coulomb failure criterion is adopted, again with mechanical properties as in Table II. The iterated solution of the optimization problem (8) allows one to estimate (lower bound approximation) the out-of-plane failure surface of the element in the $M_{x x},-M_{y y}-M_{x y}$ plane, see Figure 6.

Assuming as failure surfaces for joints and bricks those represented in Figure 5 and Figure 6, it is possible to evaluate, iteratively solving optimization problem (7), the ultimate masonry horizontal bending, vertical bending and torsion (i.e. $M_{x x}, M_{y y}$, and $M_{x y}$ ) failure surfaces to use at a structural 
level within the topology optimization strategy used to define the optimal disposition of FRP reinforcement.

The resultant failure surface is depicted in Figure 7. As it is possible to notice from the figure, $M_{x x}$ strength is higher than $M_{y y}$, which confirms the orthotropic behavior also under out-of-plane loads. Since no vertical pre-compression is present in the experimented walls, exception made for self weight which is considered negligible in the computations, failure surfaces refer to $N_{y y}=0$.

In the second example here investigated, with the aim of testing the reliability of the procedure proposed in presence of vertical pre-compression, the ultimate masonry out-of-plane strength is evaluated for a masonry wall in running bond with standard Italian bricks of dimensions $55 \times 120 \times$ $250 \mathrm{~mm}^{3}$ (height $\times$ thickness $\times$ length) and mortar joints reduced to interfaces. Bricks obey a MohrCoulomb failure criterion, whereas for joints a strength domain as in Figure 4 is assumed. Mechanical properties that fully define the strength surfaces of the constituent materials are summarized in Table III. Homogenized masonry out-of-plane failure surfaces in the $M_{x x}-M_{y y}$ and $M_{x x}-M_{x y}$ planes are depicted in Figure 8 at different values of the membrane vertical load $N_{y y}$, with the precise aim of investigating the role played by $N_{y y}$ on the homogenized out-of-plane strength. As can be observed from both Figure 8-a and -b, where respectively $M_{x x}-M_{y y}$ and $M_{x x}-M_{x y}$ sections of the failure surface are represented, when the membrane load becomes larger than 0.7-0.8 times the compression strength of joints, a drop of the out-of-plane strength is experienced.

In addition, it is interesting to notice that vertical membrane load influences not only the horizontal bending moment $\left(M_{y y}\right)$, but also the vertical one $\left(M_{x x}\right)$, as a consequence of the fact that also bed joints contribute to masonry vertical ultimate moment.

Furthermore, as experimental evidences show, there is an optimal compressive load for which failure moments reach a maximum. Exceeded this optimum point, out-of-plane strength begins to decrease until membrane compressive failure occurs. It has been demonstrated in [20] that, while a standard Mohr-Coulomb failure criterion is not able to realistically reproduce such feature, since there is an infinite strength in compression, a model with limited $f_{c}$ is able to better represent what really happens in practice, Figure 8.

When bricks are assumed infinitely resistant or when the strength of the bricks is much higher than that of the joints, masonry subjected to vertical bending $\left(M_{y y}\right)$ exhibits an ultimate out-of-plane strength ruled exclusively by the ultimate bending resistance of the joint. 
When a limited tensile and compressive strength is adopted for joints, masonry ultimate strength in vertical bending $\left(M_{y y}\right)$ is straightforwardly evaluated as follows, see also Figure 9:

$$
\begin{aligned}
& M_{y y}=\frac{x(t-x)}{2}\left(f_{c}+f_{t}\right) \\
& x=\frac{f_{t} t-N_{y y}}{f_{c}+f_{t}}
\end{aligned}
$$

Where $x$ physically represents the neutral axis of the bed joint section, $N_{y y}$ is the in plane vertical membrane action (negative if in compression), $f_{c}$ and $f_{t}$ are absolute values of mortar compressive and tensile strength, respectively.

It is interesting to compare the ultimate strength deduced from Equation (10) with the results provided by the present numerical procedure, to further assess the reliability of the model proposed in presence of vertical pre-compression. The comparison is shown in Figure 9, where ultimate vertical bending moments $\left(M_{y y}\right)$ obtained by means of either the present approach or Equation (10) are depicted. As can be noted, the correspondence is almost perfect.

\section{Topology optimization problem}

Two optimization approaches for the distribution of the optimal fiber-reinforcement for out-ofplane loaded masonry structures are herein investigated following the approach for in-plane loads, originally introduced in [17][40].

Within a two-dimensional domain $\Omega$, a linear elastic orthotropic body subjected to out-of-plane loads is considered. A reinforcing material is applied on both sides of the body, with the aim of improving stiffness and strength of the underlying masonry layer. The reinforcing layers are supposed to undergo membrane forces only, which add bending stiffness to the structure depending on their lever arm. Under the assumption of perfect bonding, a reinforcing layer can be therefore modeled as an additional in-plane stiffness contribution to the underlying brickwork. The stiffness of any finite element (i) into which the reinforced structure is subdivided can be written as:

$K_{T i}\left(x_{i, 1}, x_{i, 2}\right)=K_{M i}+x_{i, 1}^{p} K_{R i, 1}+x_{i, 2}^{p} K_{R i, 2}$,

where $K_{T i}\left(x_{i, 1}, x_{i, 2}\right)$ is the stiffness matrix of the $i$-th reinforced finite element, $K_{M i}$ is the stiffness contribution of the underlying orthotropic masonry structure, whereas $K_{R i, 1}, K_{R i, 2}$ account for an isotropic layer of reinforcement placed on the two sides of the masonry element. The latter contributions depend on the density of the reinforcement on each side of the $i$-th element, $x_{i, 1}, x_{i, 2}$ (with $0 \leq x_{i, 1}, x_{i, 2} \leq 1$ ), according to the so-called SIMP law [41]; $p=3$ in the application presented hereafter [42]. 
As introduced in Section 1, a wide literature exists that addresses the automatic generation of reinforcement layouts for structural elements. A classical application exploits methods of topology optimization to derive truss-like structures the can be interpreted as load paths, suggesting rebar of concrete elements along tensile members of the achieved optimal design, see in particular [19] [43][45]. Building codes suggest the adoption of the strain energy of the structural element as the objective function to generate admissible layouts at the ultimate limit state, see also [46]. The minimization of the strain energy for applications of fiber-reinforcement to existing structural element has been used accordingly in [47] and [18]. However, brittleness of some building materials such as brickwork suggests investigating alternative approaches taking into full account the failure criterion of the structural element to be reinforced, see [40],[17] and also [48].

Coping with the herein considered out-of-plane behavior of masonry panels, this contribution proposes a reinforcement strategy that is based on the adoption of a stress-constrained minimization of the structural weight of the reinforcement. The discrete version of the problem implemented in this work can be written as:

$$
\left\{\begin{array}{l}
\min _{x_{i, 1}, x_{i, 2}} \sum_{i=1}^{n}\left(x_{i, 1} A_{i, 1} t_{F}+x_{i, 2} A_{i, 2} t_{F}\right) \\
\text { s.t. } \\
\sum_{i}^{n}\left(K_{M i}+x_{i, 1}^{p} K_{R i, 1}+x_{i, 2}^{p} K_{R i, 2}\right) \mathbf{u}=\mathbf{f} \\
\mathbf{F}_{M}\left(\boldsymbol{M}_{M k}\right) \leq 0, \quad k=1, \ldots, m \\
0<x_{i, 1} \leq 1, \quad i=1, \ldots, n \\
0<x_{i, 2} \leq 1, \quad i=1, \ldots, n
\end{array}\right.
$$

The objective function in the above equation is the weight of the reinforcement, being $A_{i, 1}$ and $A_{i, 2}$ the areas of the $i$-th finite element, $t_{F}$ the prescribed reinforcement thickness on each side of the panel, and $n$ the number of finite elements.

The first constraint of the optimization problem in eq. (12) enforces the displacement-based equilibrium equation for the reinforced structural element in weak form. The global stiffness matrix was split into three contributions related to the underlying masonry element and the reinforcing layers, in agreement with eq. (11). The second requirement consists of a set of local constraints that enforce the strength criterion discussed in the previous section, involving the components of the moment vector $\boldsymbol{M}_{M k}$ computed in the $k$-th element of the masonry layer, see in particular [49]. For each element, more than one constraint is formulated. In fact the smooth failure surface is approximated by a set of planes that may be usefully adopted to enforce feasibility of the moments computed in each finite element discretizing the masonry layer as a set of linear inequalities. Whereas all the inequalities prescribed by the adopted strength criterion are evaluated at each finite 
element, only a few are actually implemented as effective enforcements according to [50]. This approach allows for a significant reduction in the number of active constraints, as a very limited set of local enforcements $(m<<n)$ can be selected and included in the optimization algorithm.

Since internal actions constraints are prescribed on a fixed phase of the domain, i.e. the masonry layer, the well-known singularity problem does not affect the minimization procedure, and no relaxation is required to handle internal actions constraints, see e.g. [51].

The presented optimization problem is solved by means of mathematical programming adopting the Method of Moving Asymptotes (MMA) by [52]. The adopted gradient-based optimizer is wellsuited for large scale problems, calling for the sensitivity analysis of objective function and constraints on the two sets of variables, i.e. $x_{i, 1}, x_{i, 2}$. Sensitivity computation is performed according to the adjoint method that allows reducing the computational burden of the multi-constrained formulation by fully exploiting the implemented selection strategy [53]. The starting guess for the density unknowns consists of full reinforcement prescribed for each side of the structural element, which means $x_{i, 1}, x_{i, 2}=1$ all over the domain. In the results presented in the following section, it will be found that $x_{i, 1}=x_{i, 2}$ for each finite element, as expected from the adopted modeling of the out-of-plane behavior of the reinforced masonry panel. This allows for a symmetric disposal of the strengthening layers with respect to the medium plane of the panel and is ideally conceived to resist moments induced by two-way actions such as seismic loads. However, the adoption of two independent sets of unknowns $\left(x_{i, 1}, x_{i, 2}\right)$ allows providing an ad hoc solution also in the case one side of the panel should not be available to any reinforcement.

Optimal layouts achieved by the stress-based approach of eq. (12) will be compared to results generated through the energy-based formulation reported next, see also [18]:

$\left\{\begin{array}{l}\min \mathbf{u}^{T} \sum_{i}^{n} K_{T i}\left(x_{i, 1}, x_{i, 2}\right) \mathbf{u} \\ x_{i, 1}, x_{i, 2} \\ \text { s.t. } \\ \sum_{i}^{n}\left(K_{M i}+x_{i, 1}^{p} K_{R i, 1}+x_{i, 2}^{p} K_{R i, 2}\right) \mathbf{u}=\mathbf{f} \\ \sum_{i=1}^{n}\left(x_{i, 1}+x_{i, 2}\right) A_{i} / \sum_{i=1}^{n} 2 A_{i} \leq V_{f} \\ 0<x_{i, 1} \leq 1, \quad i=1, \ldots, n \\ 0<x_{i, 2} \leq 1, \quad i=1, \ldots, n\end{array}\right.$

The objective function of the above equation is the structural compliance, that is, the discrete bilinear form computed through the local stiffness contributions in eq. (11) and the array of the nodal displacements at equilibrium, i.e. $\boldsymbol{u}$. The first constraint of the optimization problem enforces 
the equilibrium equation for the reinforced structural element in weak form, as found in eq. (12). The second enforcement represents instead a global constraint that is prescribed to the available (normalized) amount of material, that is the so-called volume fraction $V_{f}$.

\section{Case studies / numerical simulations}

A set of windowed panels, sketched in Figure 10 and labeled here from SB01 to SB03, were experimentally tested up to failure without any reinforcement by other authors [36][37]. Here, they are handled through the topology optimization algorithm presented in Section 5 to figure out a possible layout of fiber-reinforcement. The dimensions of all the panels are $5615 \times 2475 \times 102.5 \mathrm{~mm}^{3}$. Each panel was built in stretcher bond between two stiff abutments, with simply supported vertical edges (allowance for in-plane displacements was provided); the top edge was free and the bottom edge was fixed.

Running bond masonry is well known to have orthotropic mechanical properties even in the elastic range: for this reason orthotropy in the linear elastic rangehas been fully taken into account in the following simulations, although moderate effects are expected on the topology optimization results with respect to a simplified isotropic modelling, see in particular [40]. The equivalent elastic properties of masonry are $E_{x}=14534 \mathrm{MPa}, E_{y}=12420 \mathrm{MPa}, G_{x y}=4914 \mathrm{MPa}$ and $v_{x y}=0.1588$, being $x$ the horizontal axis. The above values have been derived according to the homogenization procedure presented in the previous sections, whereas results are in agreement with indications provided by both experimental and numerical literature dealing with this set of experiments (see e.g. [15]).

The presented formulation for the topology optimization of any reinforcement is implemented with the aim of distributing retrofitting material with Young modulus $E_{f}=230 \mathrm{GPa}$ and a Poisson's ratio $v_{f}=0.2$, over two layers of thickness $t_{F}=0.5 \mathrm{~mm}$ bonded at both sides of the structure.

The minimum weight solution compatible with the prescribed strength criterion, see Figure 7 , for the masonry layer acted upon by an out-of-plane load $p=0.165 \mathrm{kN} / \mathrm{m}^{2}$ is presented in Figure 11(a). Black regions stand for reinforced zones, whereas white regions for unreinforced ones. Reinforcement is mainly located along the clamped edge of the panel, in order to increase strength where maximum bending is expected, see also the relevant principal stress directions in the fibers of Figure 11(b). Figure 12 shows contour plots of the moments Mxx, Myy and Mxy, computed in the masonry layer of the unreinforced panel and their feasibility with respect to the strength criterion. The feasibility ratio, see Figure 12(d) is lower or, at most, equal to 1, meaning that the adopted stress-constrained formulation is effective in providing a robust control of the stress field all over the domain. For the sake of completeness, in Figure 13(a) the location of the centers of the elements 
where out-of-plane masonry internal actions (bending moment and torque) stand on the failure surface (critical points) is depicted. Each node is identified by a different symbol (colored spheres or Platonic solids) and the same graphical notation is used in Figure 13(b), where a representation of the failure surface with the state of stress of the same critical points is provided. As can be noted, the internal actions state falls on the failure surface, to demonstrate that the stress-based topology optimization problem has provided an admissible solution.

The layout shown in Figure 11 has a final volume fraction $V_{f}$ equal to 0.25 , meaning that $12.5 \%$ of each vertical side should be covered by the fiber-reinforcement, whereas the maximum out-of-plane deflection is $1.59 \mathrm{~mm}$. The achieved volume-fraction has been prescribed also within an energybased formulation to look for the optimal distribution of the same amount of material such that the compliance is minimized, i.e. stiffness is maximized. The optimal layout is provided in Figure 14. It mainly resembles that of Figure 11, except for the fact that the energy-based procedure gathers most of the material towards the center of lower edge of the panel, whereas the stress-based layout is nearly step-wise uniform along the edge. This minor difference has a remarkable outcome over the performances of the reinforced panels. As expected, the energy-based solution is the stiffest, as the relevant maximum out-of-plane deflection is $1.35 \mathrm{~mm}, 15 \%$ less than the stress-based solution. However, the moments arising in the masonry layer are not feasible with respect to the strength criteria, since the feasibility ratio is larger than 1 along most of the lower edge of the reinforced panel, see Figure 15.

The layouts above presented have been achieved through a discretization of 512 elements, which, although rough, is able to provide a preliminary advice for the optimal deployment of the fiberreinforcement. Figure 16(a) shows a stress-based design achieved through a discretization of 2024 elements, whereas $p=0.160 \mathrm{kN} / \mathrm{m}^{2}$. This decrease in terms of the out-of-plane load (around $3 \%$ ) is suited to provide a direct comparison with the design in Figure 11, since both share the same volume fraction $V_{f}$, but the finer discretization allows for a more accurate approximation of the stress field (with higher peaks). The optimal layout in Figure 16(a) is more or less the same presented in Figure 11 except for the minor branches found in the finer mesh. As already discussed for plane problems in [40], mesh dependence and other typical numerical instabilities can be avoided through the adoption of a conventional filter acting on the density variable throughout the optimization procedure, as done in conventional problems of topology optimization, see e.g. [54] and also [55]. Figure 16(b) shows the effect of such a regularization adopting a filtering radius that is equal to one and a half the side of the elements. It must be remarked that the stress-based layouts in Figure 11(a), Figure 16(a) and Figure 16(b) provide the designer with the same preliminary 
information about the optimal arrangement of the reinforcement, which is not so far from that achieved through an energy-based approach, see Figure 14.

Major differences between stress-based and energy-based solutions are found when coping with larger values of the applied out-of-plane pressure. Figure 17 shows the stress-based design achieved for $p=0.185 \mathrm{kN} / \mathrm{m}^{2}$. An increased area of reinforcement equal to $V_{f}=0.7$ is needed to strengthen the panel such that the failure criterion is satisfied all over the masonry layer, see Figure 18. Going into details, a horizontal strip is added at the top of the panel along with two main areas connecting the lower reinforcement with the supported vertical edges of the panel. This additional amount of reinforcement has the main aim of increasing the stiffness of the panel along the horizontal direction in order to relieve main moments acting along the vertical one.

Similarly to the previous case, in Figure 19(a) the location of the center of the elements where outof-plane internal actions on masonry layer (bending moment and torque) stand on the failure surface (critical points) is depicted. In Figure 19(b), a representation of the failure surface with the state of stress of the same critical points is provided. Again, the internal actions stands on the failure surface.

For the same volume fraction the energy-based procedure finds the optimal layout in Figure 20. Except for the lower strip of reinforcement, the achieved design is completely different for the previous one, since additional fibers are distributed in the upper part of the panel, mainly along its vertical edges. As expected, the energy-based solution is stiffer than the stress-based (1.27 mm vs $1.32 \mathrm{~mm}$ of out-of-plane deflection), but the moments arising in the masonry layer are not feasible with respect to the stress criterion, see in particular Figure 21(d).

A final investigation on this example refers to numerical issues. Figure 22 compares the history plot of the stress-based procedure launched over the mesh of 512 elements for the two investigated values of the out-of-plane load, $p=0.160 \mathrm{kN} / \mathrm{m}^{2}$ and $p=0.185 \mathrm{kN} / \mathrm{m}^{2}$, both in terms of weight of the reinforcement and number of active constraints. Smooth convergence of the objective function is observed, with extended plateaux starting form the first 10/15 iterations. The adoption of a full material distribution as starting guess allows reducing the computational burden peculiar to the first iterations of the multi-constrained optimization, see in particular [50]. After the peak of these steps, the selection strategy chooses a limited amount of active local constraints (less than 15\% of the elements in the mesh). Their number is stable throughout the optimization procedure and is nearly equal for both values of the out-of-plane loads. In fact, independently on the amount of distributed reinforcement, the set of finite elements governing the failure of the masonry layer is approximately the same: all of them are located within the middle lower region of the panel, see in particular Figure 12(d) and Figure 18(d). 
The windowed panel of Figure 10(b), SB02, is investigated over a mesh of 674 finite elements, looking for the best distribution of reinforcement to improve its response to out-of-plane loads. Figure 23 shows the stress-based solution for $p=0.250 \mathrm{kN} / \mathrm{m}^{2}$, whereas Figure 24 shows contour plots of the moments in the masonry layer that are fully compliant with the strength criterion. Looking at Figure 24(d) one may easily notice that the big hole in the center of the panel extends the size of the critical region along the clamped edge, whereas stress concentrations arise in the vicinity of its lower corners. Undesired stress peaks are controlled through an ad hoc local reinforcement located next to the geometric singularities, whereas moments acting in the vertical directions are released increasing the stiffness of the lintel. As done for panel SB01, in Figure 25(a) the location of the nodes where out-of-plane internal actions on masonry layer (bending moment and torque) stand on the failure surface (critical points) is depicted, whereas in Figure 25(b) a representation of the masonry failure surface with the state of stress of the same critical points is provided.

Figure 26 shows the energy-based solution that has been achieved for the same amount of reinforcement arranged by the stress-based solution, i.e. for $V_{f}=0.4$. The solution is almost similar to that represented in Figure 23. As expected, it performs slightly better referring to the overall stiffness, but worst concerning feasibility of the moments in the masonry layer with respect to the strength criterion, see Figure 27.

The same investigation is performed adopting a finer mesh of 2696 finite elements. Figure 28 shows a comparison of a stress-based design, whose pressure load $p=0.225 \mathrm{kN} / \mathrm{m}^{2}$ has been chosen in order to achieve $V_{f}=0.4$, along with an energy-based design enforcing the same amount of fiberreinforcement. These finer layouts increase readability of the results achieved on the coarser mesh and assess that minor differences arise between a strength-driven optimization and its energy-driven counterpart. As discussed referring to the panel SB01, this is observed if a low volume fraction is arranged over the masonry layer, while significant variations are expected for a higher amount of reinforcement to be distributed.

Figure 29 shows the geometric location of the center of the critical elements where the internal actions stand on the failure surface adopted for masonry, with the same symbols (spheres and Platonic solids) used for the previous examples.

Figure 30(a) depicts the optimal solution found for the pressure load $p=0.240 \mathrm{kN} / \mathrm{m}^{2}$, accounting for a volume fraction of $V_{f}=0.7$, whereas Figure 30(b) allows assessing the full feasibility of the achieved layout with respect to the prescribed strength criterion. As a variation of Figure 28, the optimal design for the increased load pressure additionally strengthens the panel along the 
horizontal direction through a direct connection between the lower corner of the hole and the simply supported edges of the panel.

Figure 31 shows the geometric location of the center of the critical elements assuming $p=0.240 \mathrm{kN} / \mathrm{m}^{2}$, with an indication of their position on the failure surface.

For the same volume fraction, the optimal energy-based design is represented in Figure 32(a). The design is significantly different in the distribution of the additional reinforcement within the lateral regions of the panel. This result performs better in terms of stiffness, but moments are not feasible with respect to the strength criterion, exceeding the threshold of about 25\%, see Figure 32(b).

The stress-based optimization routines launched for panel SB02 over the mesh accounting for 2696 elements have been used to provide a comparison in terms of history plots of the objective function and number of active constraints. As already observed about a coarser mesh for the panel SB01, convergence is smooth and the number of active constraint is approximately the same notwithstanding the different amount of reinforcement to be distributed depending on the applied pressure load, see Figure 33. For both the minimization procedures that have been herein considered for panel SB02, the number of local enforcements is remarkably lower than the number of finite elements (less than 20\%) during most of the optimization. The proposed procedure allows for a robust control of the internal actions field, while mitigating the computational burden tied to multiconstrained formulations.

A final set of simulations is performed on the panel SB03 of Figure 10(c) to investigate the effect of a door opening within the wall. Figure 34 represents the stress-based design achieved for an out-ofplane load equal to $p=0.155 \mathrm{kN} / \mathrm{m}^{2}$, calling for the low volume fraction $V_{f}=0.35$. As shown by the principal stress directions found in the optimal reinforcement layer, fibres at the clamped edge of the panel provide stiffness and strength along the vertical direction, while the reinforcement of the lintel serves as a strengthening of the load transfer along the horizontal direction. This arrangement avoids the failure of the panel, see Figure 35, but it is not expected to be the best solution in terms of stiffness.

Figure 36 shows the energy-based design achieved for the same volume fraction of material that matches this performance, but is unfeasible with respect to the prescribed failure criterion, see Figure 37(d). Although the main layout of the two aforementioned solutions is almost the same, some difference arises in the arrangement of the reinforcement at the bottom of the panel. As already found for panel SB01, the energy-based procedure distributes a larger amount of fibres next to the middle of the panel, whereas the stress-based minimization provides the lower strip of reinforcement with a more homogeneous thickness. 
It must be remarked that all the simulations based on the stress-constrained formulation have been performed enforcing a step-wise linear approximation of the failure criterion involving 24 planes. To this purpose, Figure 38 shows the geometric location of the center of critical elements in this latter case, with an indication of their position on the out-of-plane failure surface adopted. An increased number of inequalities could be similarly employed to provide a finer approximation of the failure surface, with the remarkable counterpart of increased computational costs. Having the aim of investigating pros and cons of such a strategy, the stress-based optimization for the case study of panel SB03 has been repeated adopting 72 planes and 144 planes, respectively.

The positions of the critical elements in these latter cases with the indication of their state of stress (with the same symbols adopted previously) are represented in Figure 39 and Figure 40 for the failure surfaces with 72 and 144 planes, respectively. As can be noted, the results found are very similar to those obtained with the coarse approximation by means of 24 planes.

In addition, the design of Figure 34 has been achieved by both simulations, as assessed by Figure 41(a). The same weight is found at convergence, but it requires a number of iterations that slightly increases for an increased number of planes. Figure 41(b) points out that a finer approximation of the failure surface calls for a wider set of active enforcements throughout the optimization procedure. The size of this set is mainly responsible for the overall cost of the multi-constrained minimization procedure. Based on the achieved results, the 24-planes approximation provides a robust design with a limited computational burden.

\section{Conclusions}

A stress-based approach has been presented for the automatic generation of optimal layouts of fiberreinforcement for masonry panels subject to out-of-plane loads. The proposed algorithm arranges the minimum amount of material that is needed to allow the internal actions field of the underlying masonry layer be feasible with respect to the adopted homogenized strength criterion. A multiconstrained formulation arises that is used to enforce locally the feasibility of the internal actions field through mathematic programming. To mitigate the computational burden tied to this kind of formulations a selection strategy originally developed for problems of topology optimization has been herein specialized to problems of optimal reinforcement. Numerical simulations assess the robustness of the proposed procedure, also with respect to different step-wise linear approximations of the adopted strength criterion. Optimal layouts have been compared with conventional solutions based on the minimization of the overall strain energy. Differences between the two formulations 
are minor when a low amount of fiber-reinforcement is arranged over the structure, that is $V_{f}<0.5$. If the out-of-plane loads call for higher percentage of reinforcement, an energy-based layout is quite different with respect to a stress-based design distributing the same amount of material. The conventional minimization of the strain energy can fail in avoiding the structural collapse due to moments in the masonry layer that can be larger than those allowed up to $20 \%$.

\section{References}

[1] Spence R, Coburn A. Strengthening building of stone masonry to resist earthquakes. Meccanica 1992;27:213-21.

[2] Milani G., Zuccarello F.A., Olivito R.S., Tralli A. (2007). Heterogeneous upper-bound finite element limit analysis of masonry walls out-of-plane loaded. Computational Mechanics, 40 (6), pp. 911-931.

[3] Zuccarello F.A., Milani G., Olivito R.S., Tralli A. (2009). A numerical and experimental analysis of unbonded brickwork panels laterally loaded. Construction and Building Materials, 23(5), pp. 2093-2106.

[4] Casolo S, Milani G. A simplified homogenization-discrete element model for the non-linear static analysis of masonry walls out-of-plane loaded. Eng Struct 2010;32(8):2352-66.

[5] Milani G, Lourenço PB, Tralli A. Homogenization approach for the limit analysis of out-ofplane loaded masonry walls. J Struct Eng ASCE 2006;132(10): 1650-63.

[6] De Felice G. Out-of-plane seismic capacity of masonry depending on wall section morphology. Int J Arch Herit 2011; 5(4):466-482.

[7] Milani G, Lourenço PB. A simplified homogenized limit analysis model for randomly assembled blocks out-of-plane loaded. Comput Struct 2010;88:690-717.

[8] Foraboschi P. Strength assessment of masonry arch retrofitted using composite reinforcements. Masonry Int 2001; 15(1):17-25.

[9] Grande E, Milani G, Sacco E. Modelling and analysis of FRP-strengthened masonry panels. Engng Struct 2008; 30(7):1842-60. 
[10] Capozucca R. Experimental FRP/SRP-historic masonry delamination. Compos Struct 2010; 92:891-903.

[11] Shrive NG. The use of fibre reinforced polymers to improve seismic resistance of masonry. Constr Build Mat 2006; 20(4):269-77.

[12] Baratta A, Corbi O. Stress analysis of masonry vaults and static efficacy of FRP repairs. Int J Solids Struct 2007; 44(24):8028-56.

[13] Oliveira D, Basilio I, Lourenço PB. Experimental behavior of FRP strengthened masonry arches. J Compos Constr 2010; 14(3):312-22.

[14] Borri A, Castori G, Corradi M. Intrados strengthening of brick masonry arches with composite materials. Composites Part B: Engineering 2011; 42(5):1164-72.

[15] Milani G. Homogenized limit analysis of FRP-reinforced masonry walls out-of-plane loaded. Comput Mech 2009; 43(5):617-39.

[16] Krevaikas TD, Triantafillou TC. Computer-aided strengthening of masonry walls using fibrereinforced polymer strips. Mater Struct 2005; 38:93-8.

[17] Bruggi M, Milani G, Taliercio A. Design of the optimal fiber-reinforcement for masonry structures via topology optimization. Int J Solids Struct 2013; 50(13):2087-106.

[18] Bruggi M, Milani G, Taliercio A. Simple topology optimization strategy for the FRP reinforcement of masonry walls in two-way bending. Computers \& Structures 2014; 138C: 86-101.

[19] Bruggi M. On the automatic generation of strut and tie patterns under multiple load cases with application to the aseismic design of concrete structures. Adv Struct Eng 2010; 13:1167-81.

[20] Milani G. Simple lower bound limit analysis homogenization model for in- and out-of-plane loaded masonry walls. Constr Build Mat 2011; 25:4426-43.

[21] Milani G, Lourenço PB, Tralli A. Homogenised limit analysis of masonry walls. Part I: failure surfaces. Comput Struct 2006; 84(3-4):166-80. 
[22] Pande GN, Liang JX, Middleton J. Equivalent elastic moduli for brick masonry. Comput Geotech 1989; 8:243-65.

[23] Pietruszczak S, Niu X. A mathematical description of macroscopic behavior of brick masonry. Int J Solids Struct 1992; 29(5):531-546.

[24] Anthoine A. Derivation of the in-plane elastic characteristics of masonry through homogenization theory. Int. J. Solids Struct. 32(2), 137-163, 1995.

[25] Pegon P, Anthoine A. Numerical strategies for solving continuum damage problems with softening: application to the homogenisation of masonry. Comput Struct 1997; 64(1-4):62342.

[26] Zucchini A, Lourenço PB. A micro-mechanical model for the homogenization of masonry. Int J Solids Struct 2002; 39(12):3233-55.

[27] Milani G. Simple homogenization model for the non-linear analysis of in-plane loaded masonry walls. Comput Struct 2011; 89:1586-1601.

[28] Cecchi A, Milani G, Tralli A. A Reissner-Mindlin limit analysis model for out-of-plane loaded running bond masonry walls. International Journal of Solids and Structures 2007; 44(5): 1438-1460.

[29] Cecchi A, Milani G, Tralli A. Validation of analytical multiparameter homogenization models for out-of-plane loaded masonry walls by means of the finite element method. ASCE J Eng Mech 2005; 131(2):185-98.

[30] Mistler M, Anthoine A, Butenweg C. In-plane and out-of-plane homogenisation of masonry. Comput Struct 2007; 85(17-18):1321-30.

[31] Milani G, Tralli A. Simple SQP approach for out-of-plane loaded homogenized brickwork panels accounting for softening. Comput Struct 2011; 89(1-2):201-15.

[32] Hellan K. Analysis of elastic plates in flexure by a simplified finite element method. Acta Polytech. Scand. 1967; 46:1-28.

[33] Herrmann LR. Finite element bending analysis for plates. J Engrg Mech Div 1967; 93:13-26. 
[34] Krenk S, Damkilde L, Hyer O. Limit analysis and optimal design of plates with equilibrium elements. ASCE J Eng Mech 1994; 120(6):1237-54.

[35] Krabbenhoft K, Damkilde L. Lower bound limit analysis of slabs with nonlinear yield criteria. Comput Struct 2002; 80:2043-57.

[36] Chong VL, Southcombe C, May IM. The behaviour of laterally loaded masonry panels with openings. In: Proc 3rd Int Masonry Conf, The British Masonry Society, London, 1992, p. 178-82.

[37] Southcombe C, May IM, Chong VL. The behaviour of brickwork panels with openings under lateral load. In: Proc 4th Int Masonry Conf, The British Masonry Society, London, 1, 1995; p. 105-110.

[38] Lourenço PB. On the use of homogenisation techniques for the analysis of masonry structures. Masonry Int 1997; 11(1):26-32.

[39] Casolo S., Milani G. (2013). Simplified out-of-plane modeling of three-leaf masonry walls accounting for the material texture. Construction \& Building Materials, 40, pp. 330-351.

[40] Bruggi M, Taliercio A. Topology optimization of the fiber-reinforcement retrofitting existing structures, Int. J. Solids Struct. 50 (2013) 121-136.

[41] Bendsøe M, Kikuchi N. Generating optimal topologies in structural design using a homogenization method. Comp Meth Appl Mech Eng 1988; 71:197-224.

[42] Bendsøe MP, Sigmund O. Topology Optimization - Theory, Methods and Applications. Springer, Berlin, 2003.

[43] Ali MA, White RN. Automatic generation of truss model for optimal design of reinforced concrete structures. ACI Struct. J. 2001; 98(4): 431-442.

[44] Biondini, F, Bontempi, F, Malerba, PG. Stress path adapting strut-and-tie models in cracked and uncracked R.C. elements. Struct. Eng. Mech. 2001; 12(6): 685-698.

[45] Liang QQ, Xie YM, Steven GP. Topology optimization of strut-and-tie models in reinforced concrete structures using an evolutionary procedure. ACI Struct Jour 2000; 97:322-30. 
[46] Gaynor AT, Guest JK, Moen CD. Reinforced concrete force visualization and design using bilinear truss-continuum topology optimization. J Struct Eng 2013; 139: 607-618.

[47] Cunha J., Chaves L.P., The use of topology optimization in disposing carbon fiberreinforcement for concrete structures, Struct Multidiscip Optim, 2014 (in press) DOI 10.1007/s00158-013-1036-0

[48] Steven GP, Li Q, Xie YM. Multicriteria optimization that minimizes maximum stress and maximizes stiffness. Comput Struct 2002; 80: 2433-2448.

[49] Duysinx P, Bendsøe MP. Topology optimization of continuum structures with local stress constraints. Int J Numer Methods Eng 1998, 43(8): 1453-1478.

[50] Bruggi M, Duysinx P. A stress-based approach to the optimal design of structures with unilateral behavior of material or supports. Struct Multidiscip Optim 2013; 48:311-26.

[51] Cheng GD, Guo X. e-relaxed approach in structural topology optimization. Structural and Multidisciplinary Optimization 1997; 13(4): 258-266

[52] Svanberg K. Method of moving asymptotes - A new method for structural optimization. Int J Num Methods Eng 1987; 24: 359-73.

[53] Duysinx P, Sigmund O. New developments in handling stress constraints in optimal material distributions. In: Proceedings of 7th AIAA/USAF/NASA/ISSMO symposium on multidisciplinary design optimization, AIAA, 1998, Saint Louis, Missouri, AIAA Paper 984906.

[54] Sigmund O, Petersson J. Numerical instabilities in topology optimization: A survey on procedures dealing with checkerboards, mesh-dependencies and local minima, Structural optimization 1998; 16: 68-75.

[55] Bruggi M. On the solution of the checkerboard problem in mixed-FEM topology optimization, Comput. Struct 2008; 86: 1819-1829. 


\section{Figures}
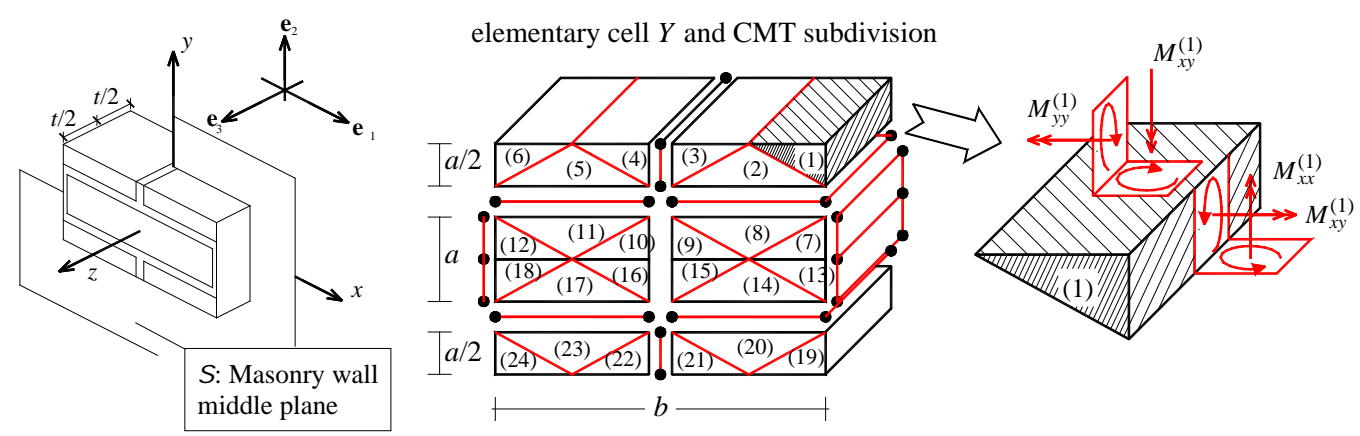

(a)

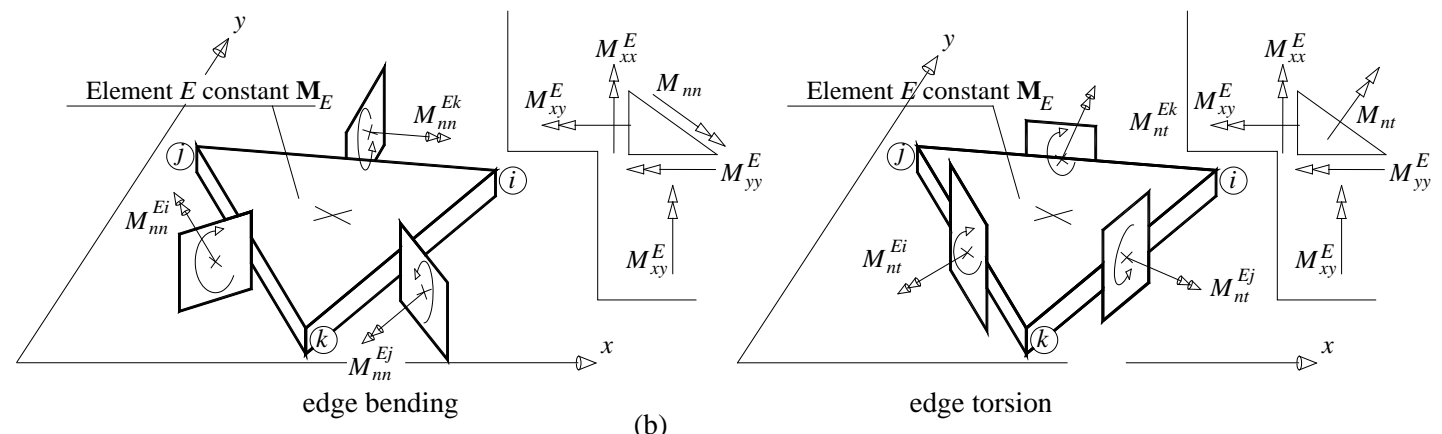

(b)

Figure 1: The micro-mechanical model proposed. -a: Subdivision of the RVE into 24 constant moment triangular elements (and 1/4 into 6 elements). -b: constant moment element, edge bending and twisting moments. 

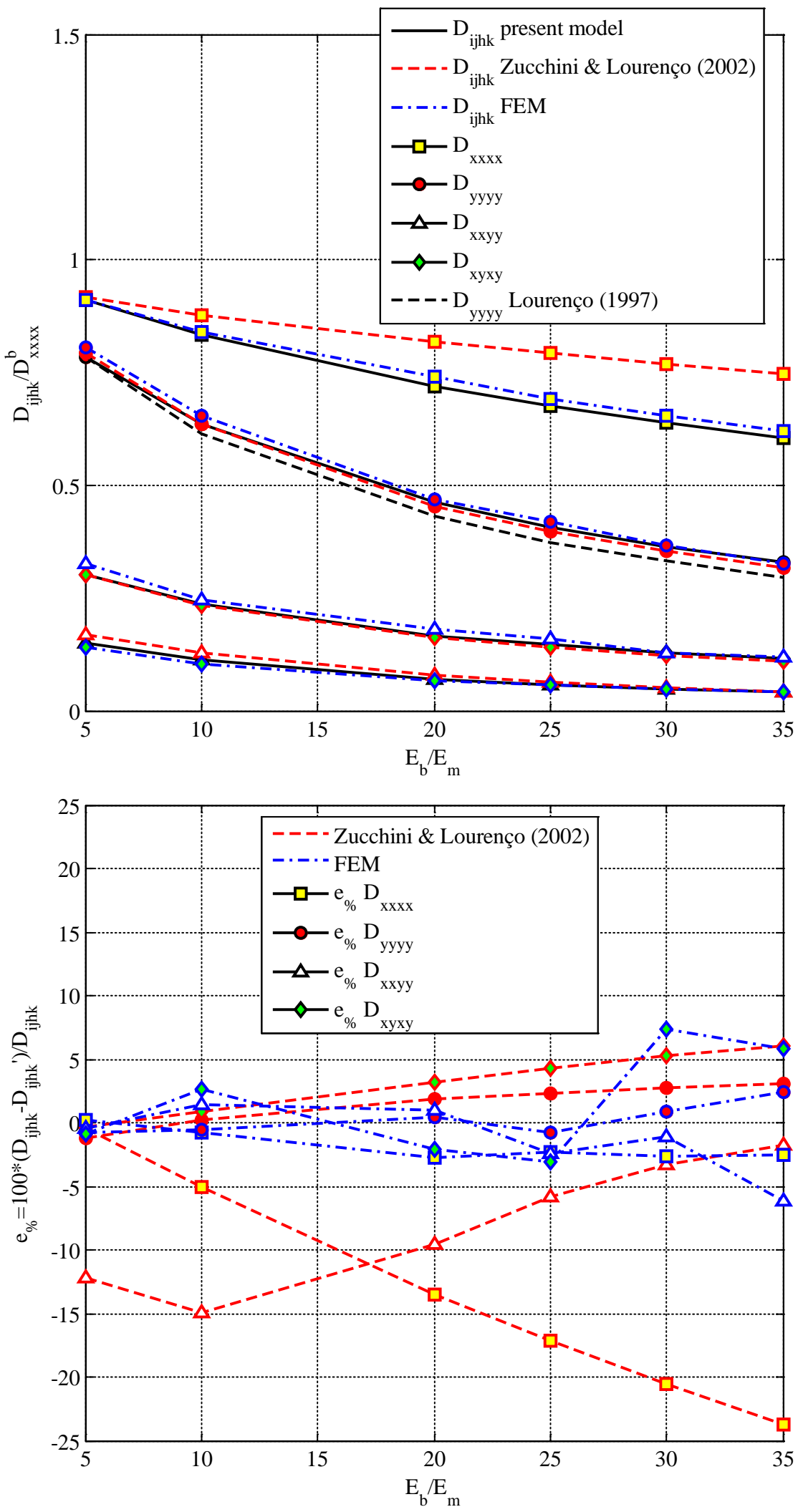

Figure 2: Homogenized out-of-plane moduli. -a: comparison between present plate approach, in-plane model available in the literature [26] and out-of-plane FEM homogenization [29]. -b: error estimation against literature model. $\mathrm{D}_{\mathrm{ijhk}}$ ' is the homogenized module by either Zucchini \& Lourenço [26] or out-of-plane FEM [29]. 

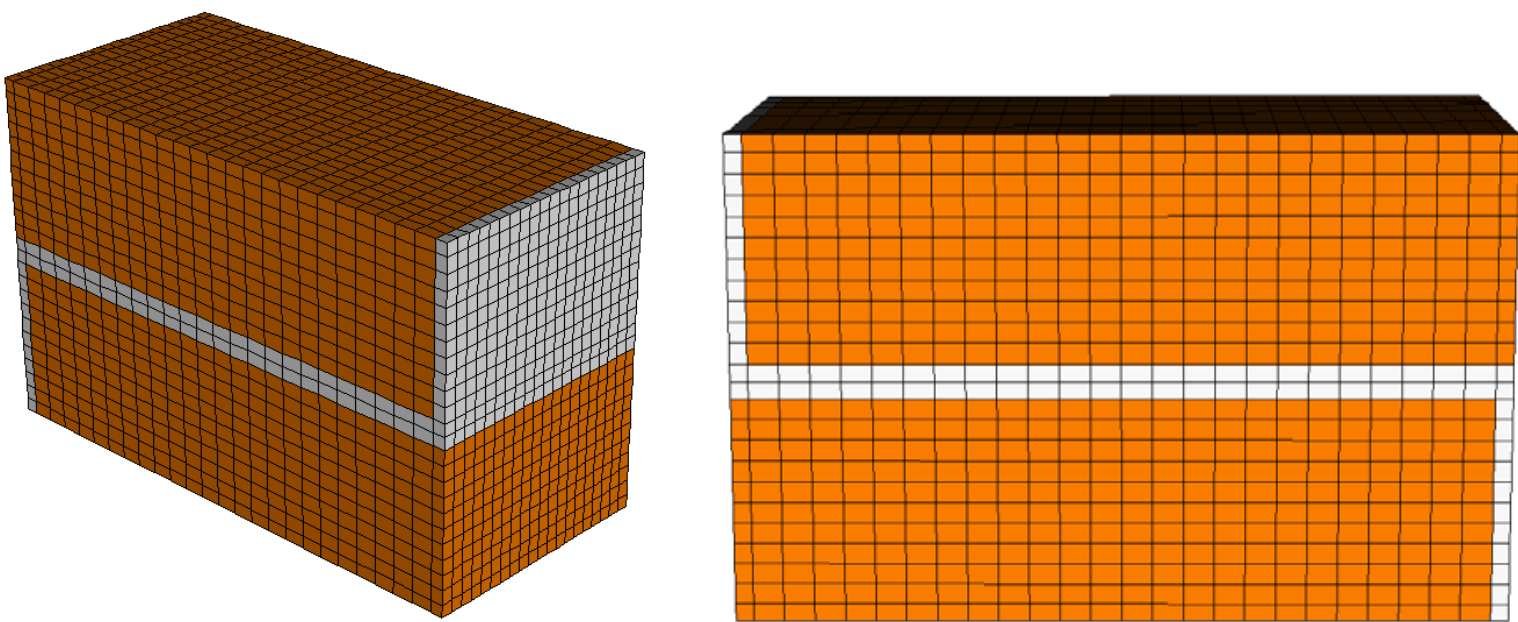

Figure 3: FE discretization of 1/8 of the elementary cell used to perform the elastic homogenized analysis, Chen and co-workers masonry (12480 bricks, 14175 nodes)

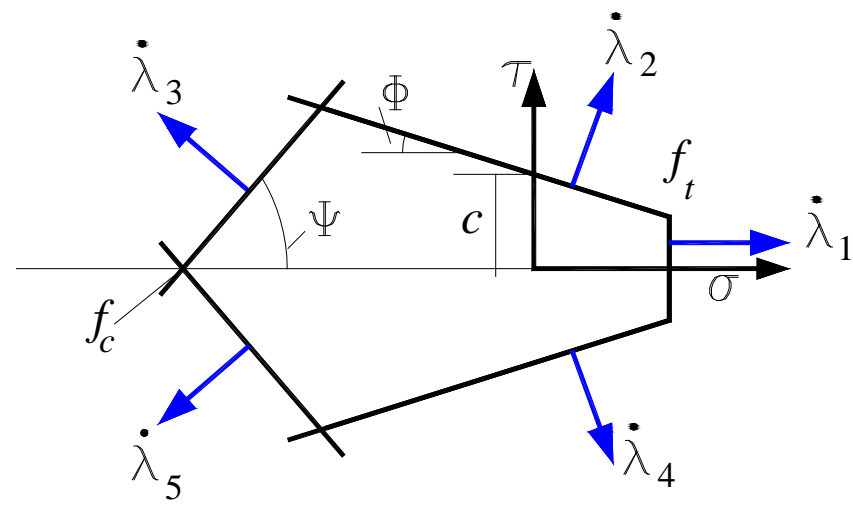

Figure 4: Linearized Mohr-Coulomb failure criterion with tension cutoff and compressive cap for mortar joints reduced to interface 

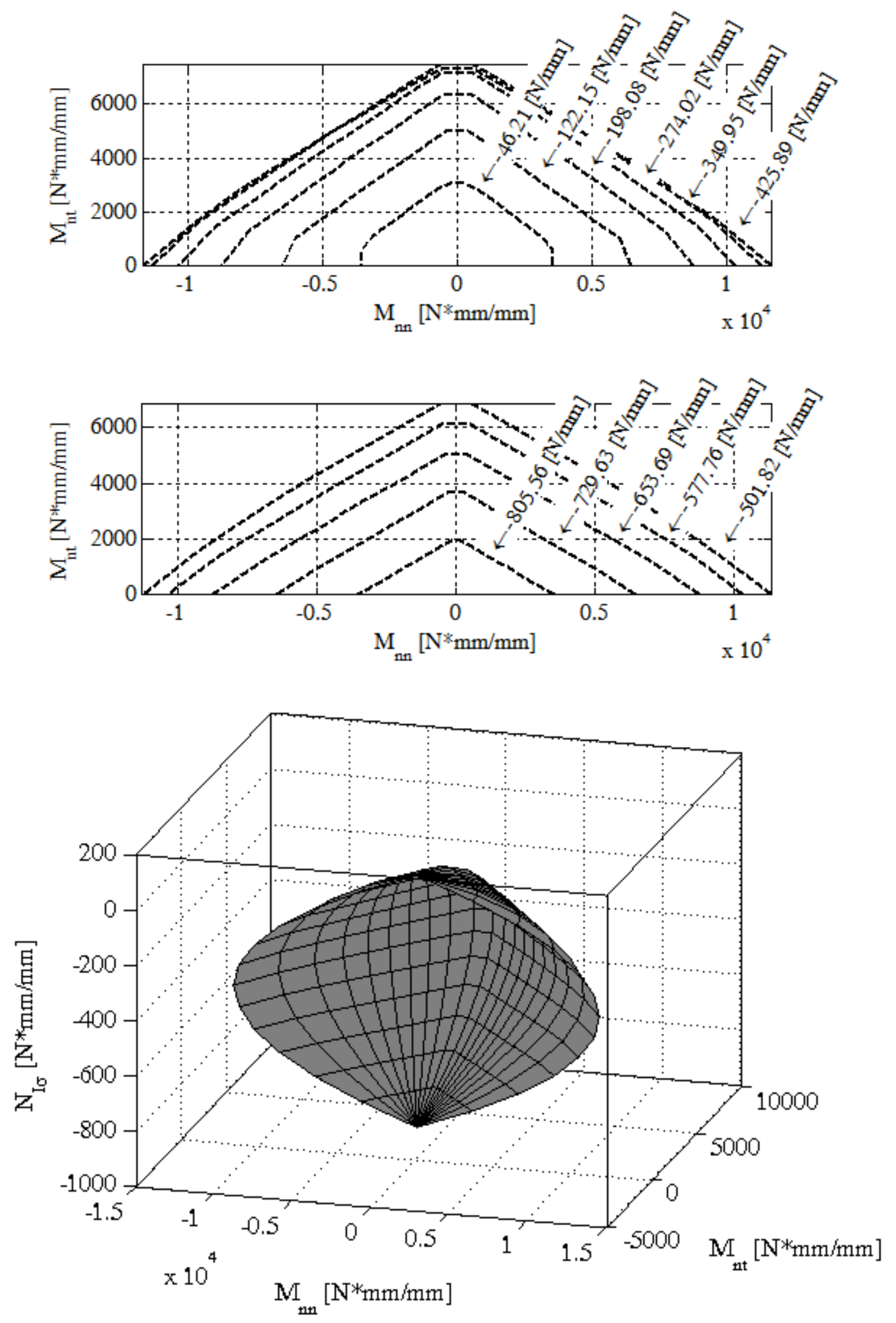

Figure 5: Out-of-plane failure criterion adopted for joint interfaces. -a: $M_{\mathrm{nn}}-M_{\mathrm{nt}}$ plane. -b: 3D space 

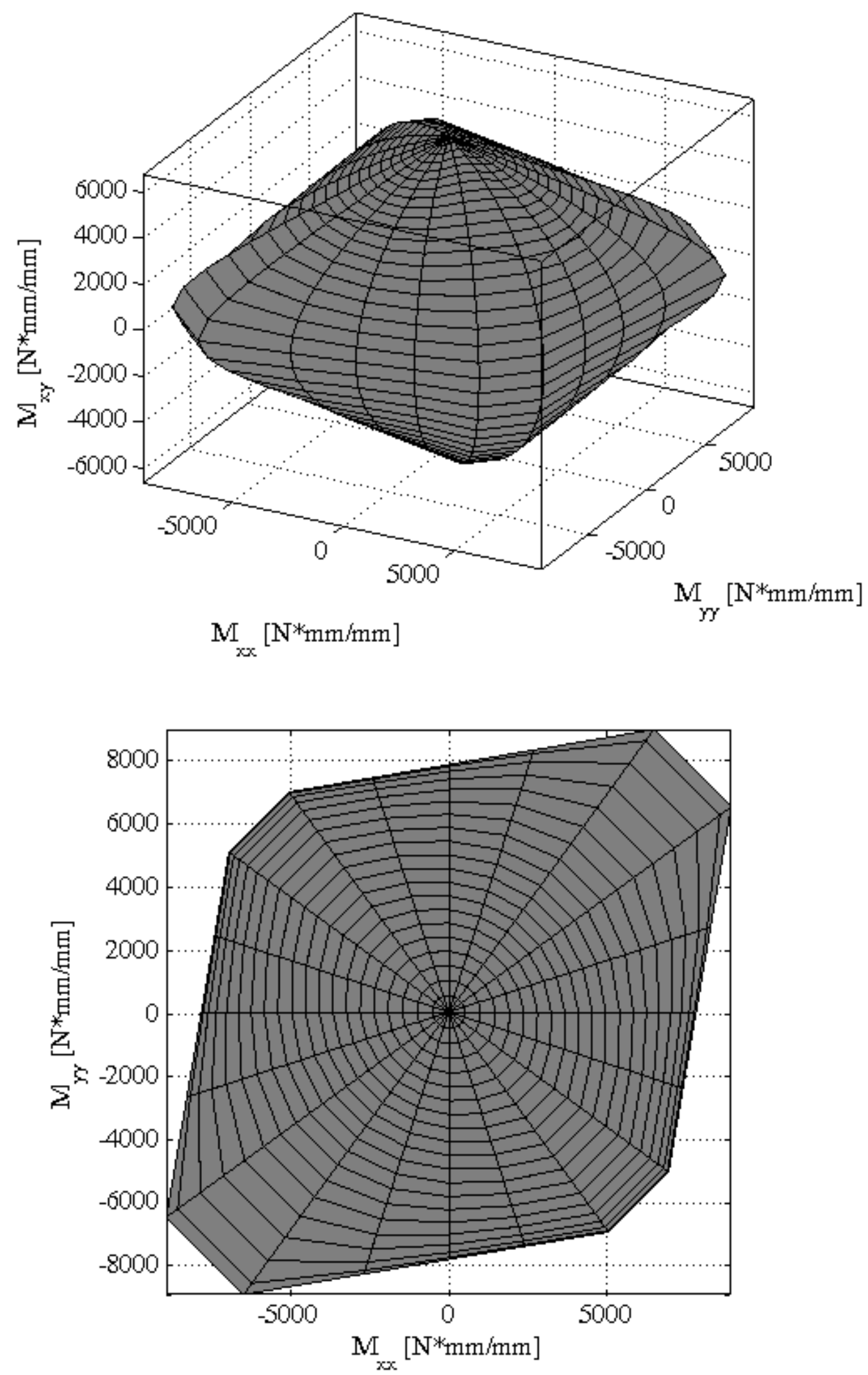

-b

Figure 6: Out-of-plane failure criterion adopted for triangular elements (brick).-a: 3D space. -b: $M_{\mathrm{xx}}-M_{\mathrm{yy}}$ plane. 


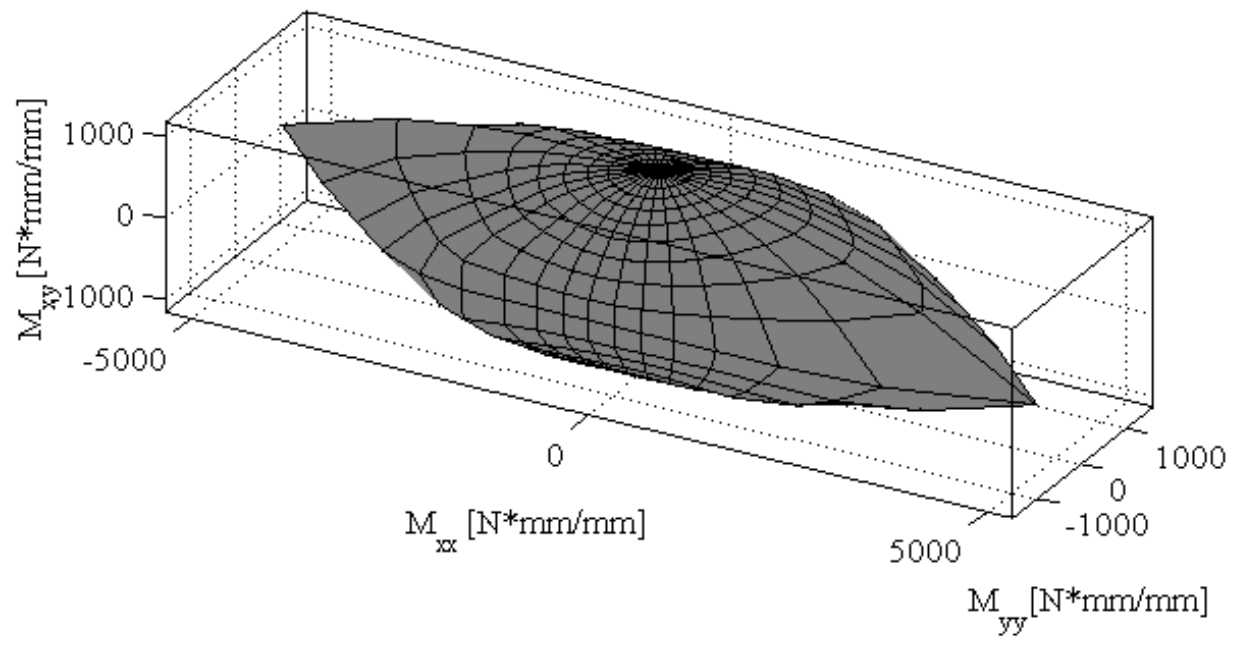

Figure 7: Homogenized out-of-plane failure surface obtained for the studied masonry. 

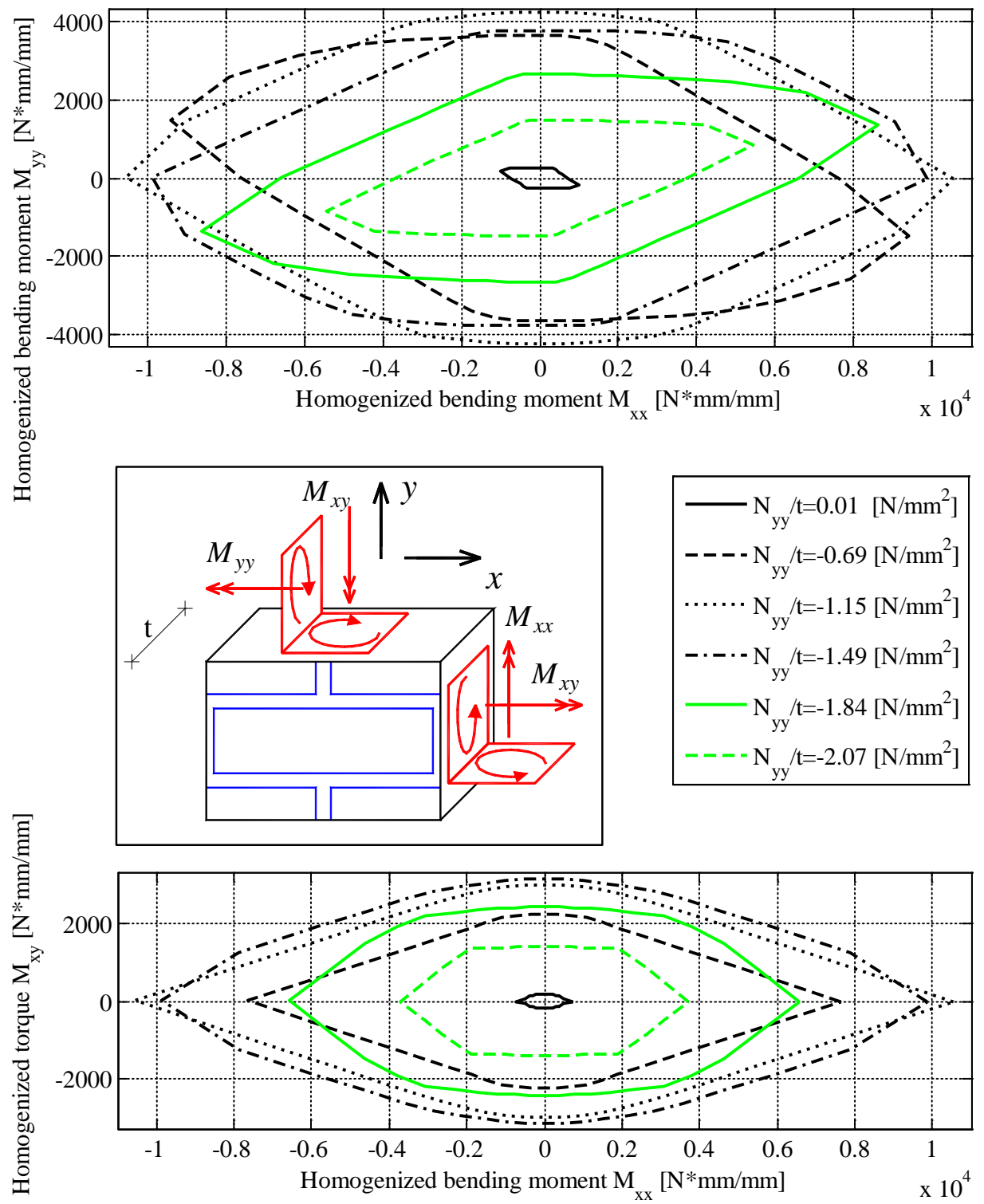

Figure 8: Failure surface obtained at increasing value of the vertical membrane load. a: $M_{x x}-M_{y y}$ Sections. $-\mathrm{b}: M_{x x}-M_{x y}$ Sections. 

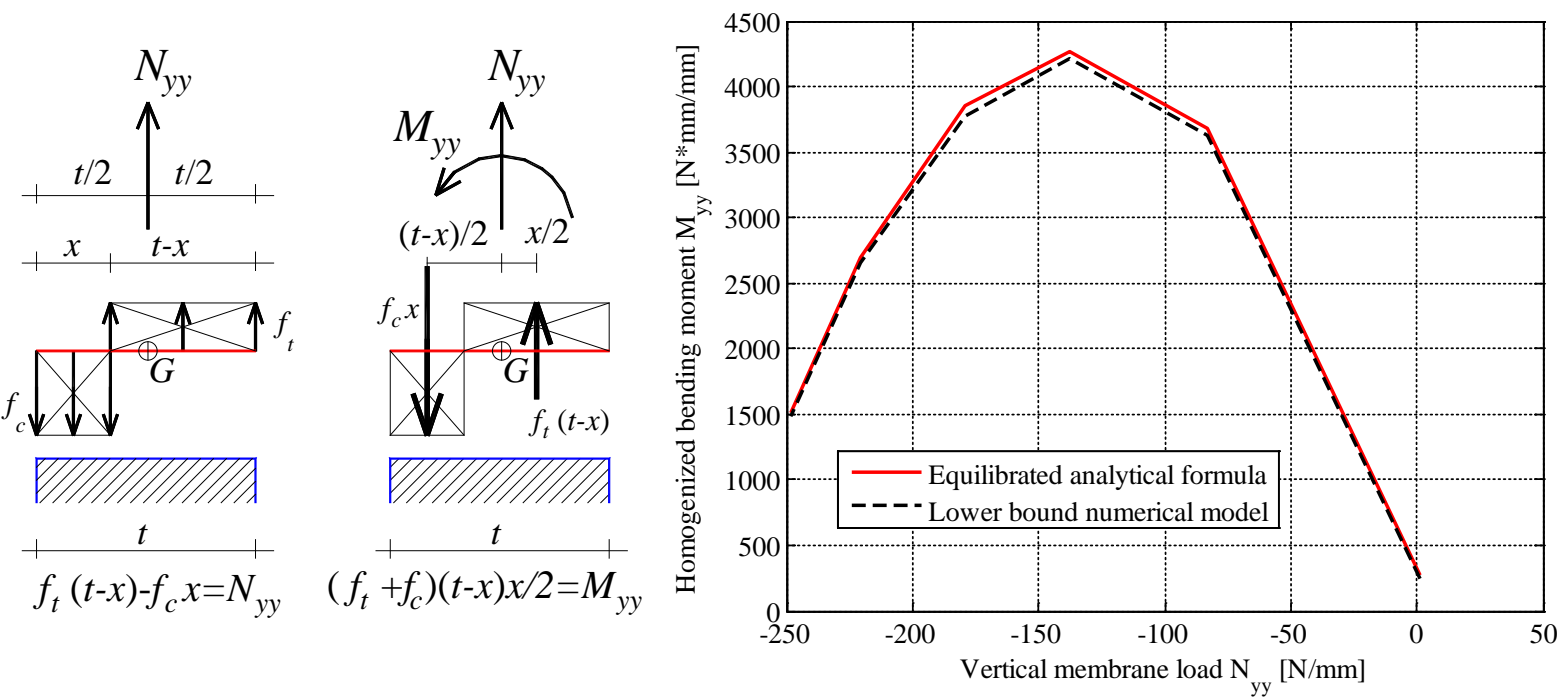

Figure 9: Left: Static approach (equilibrated analytical formula) to determine the ultimate vertical bending strength $M_{y y}$ of a masonry wall in presence of a vertical membrane load $N_{y y}$. Right: comparison between the equilibrated numerical model proposed and the equilibrated analytical formula. 

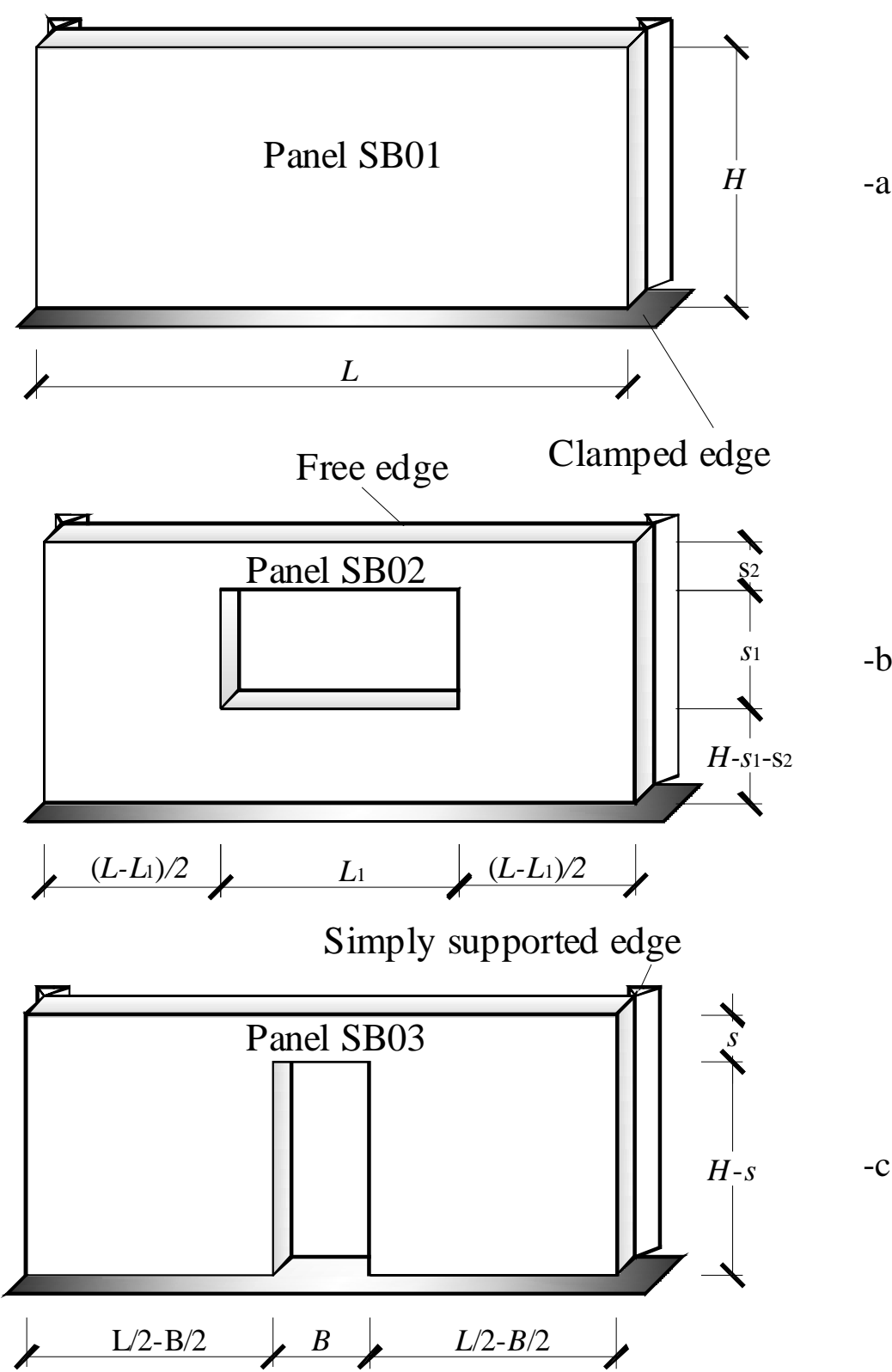

Figure 10: Windowed panels loaded out-of-plane. Dimensions of the panels and boundary conditions [36][37]. L=5615 mm H=2475 mm $\mathrm{s} 1=1125 \mathrm{~s} 2=\mathrm{s}=450 \mathrm{~mm} \mathrm{~B}=910 \mathrm{~mm} \mathrm{~L}=2260 \mathrm{~mm}$. $-\mathrm{a}$ : panel SB01. $-\mathrm{b}$ : panel SB02. - c: panel SB03. 


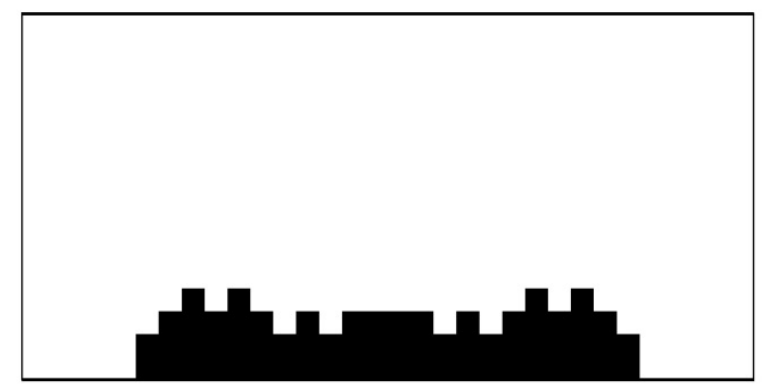

(a)

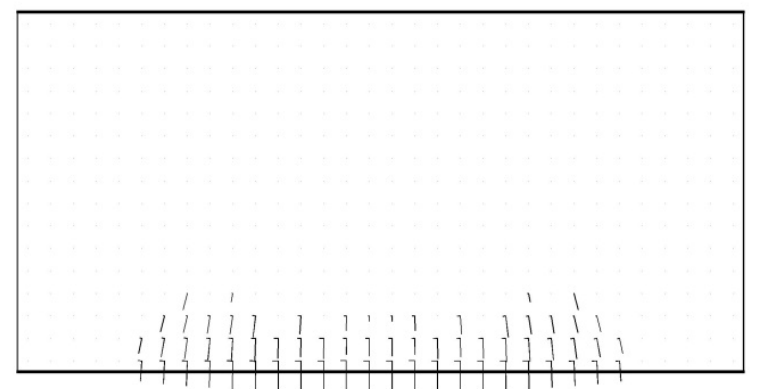

(b)

Figure 11: Panel SB01. Stress-based design for $p=0.165 \mathrm{kN} / \mathrm{m}^{2}$. Optimal distribution of the fiberreinforcement (a) and relevant principal stress directions (b), $V_{f}=0.25, w=1.59 \mathrm{~mm}$.

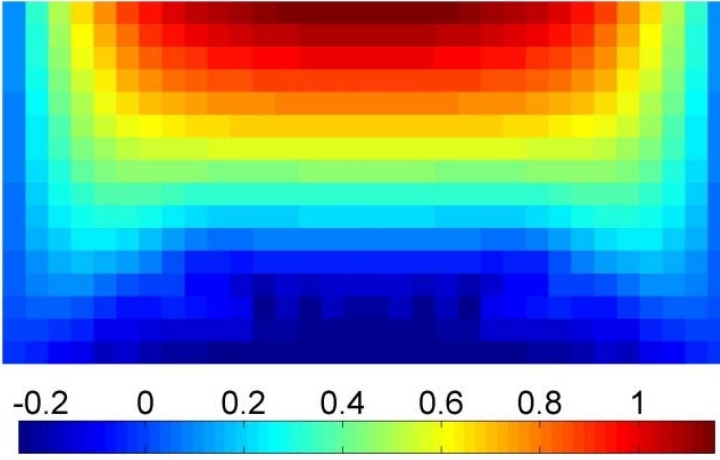

(a) $M_{x x}$

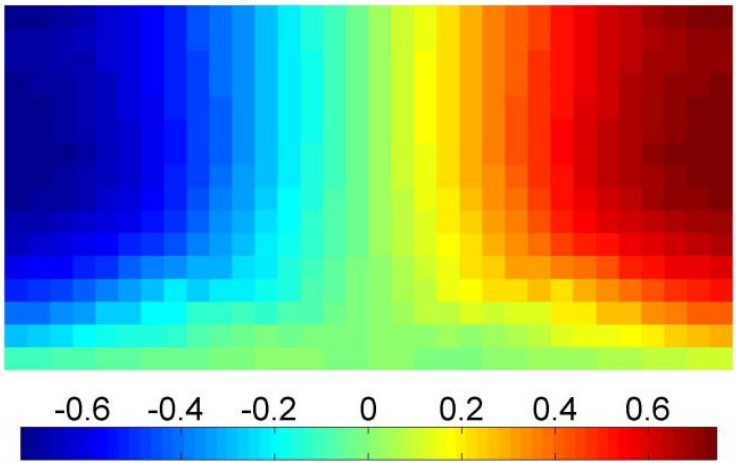

(c) $M_{x y}$

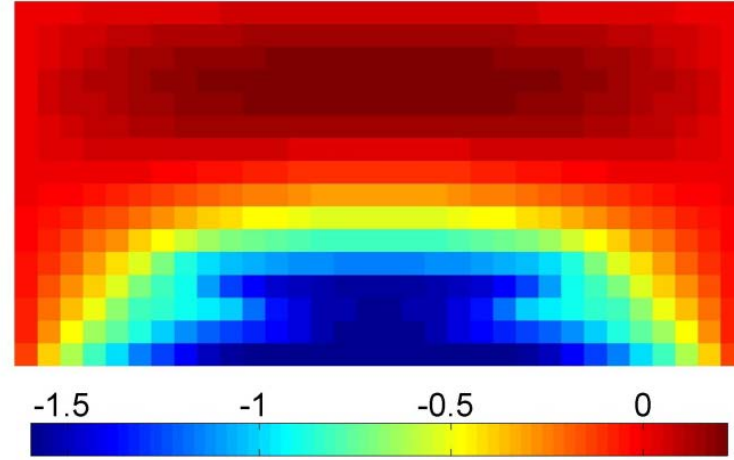

(b) $M_{y y}$

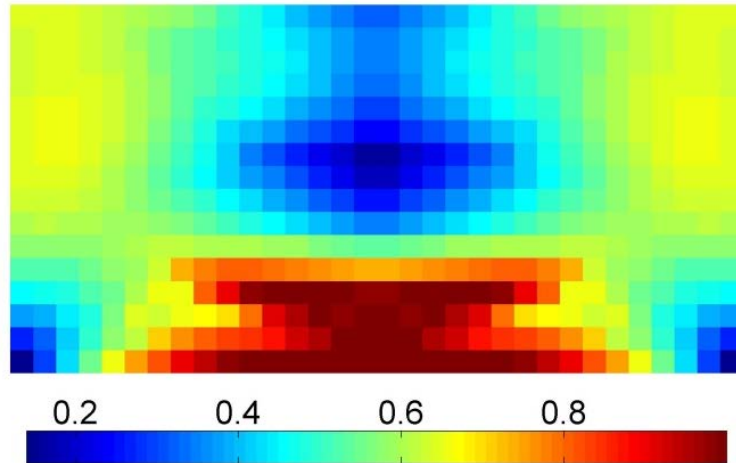

(d)

Figure 12: Panel SB01. Moments (in $\mathrm{kNm} / \mathrm{m}$ ) in the masonry layer of the panel reinforced through the stress-based optimization for $p=0.165 \mathrm{kN} / \mathrm{m}^{2}$ (a-c) and feasibility of the moment field with respect to the strength criterion (d). 

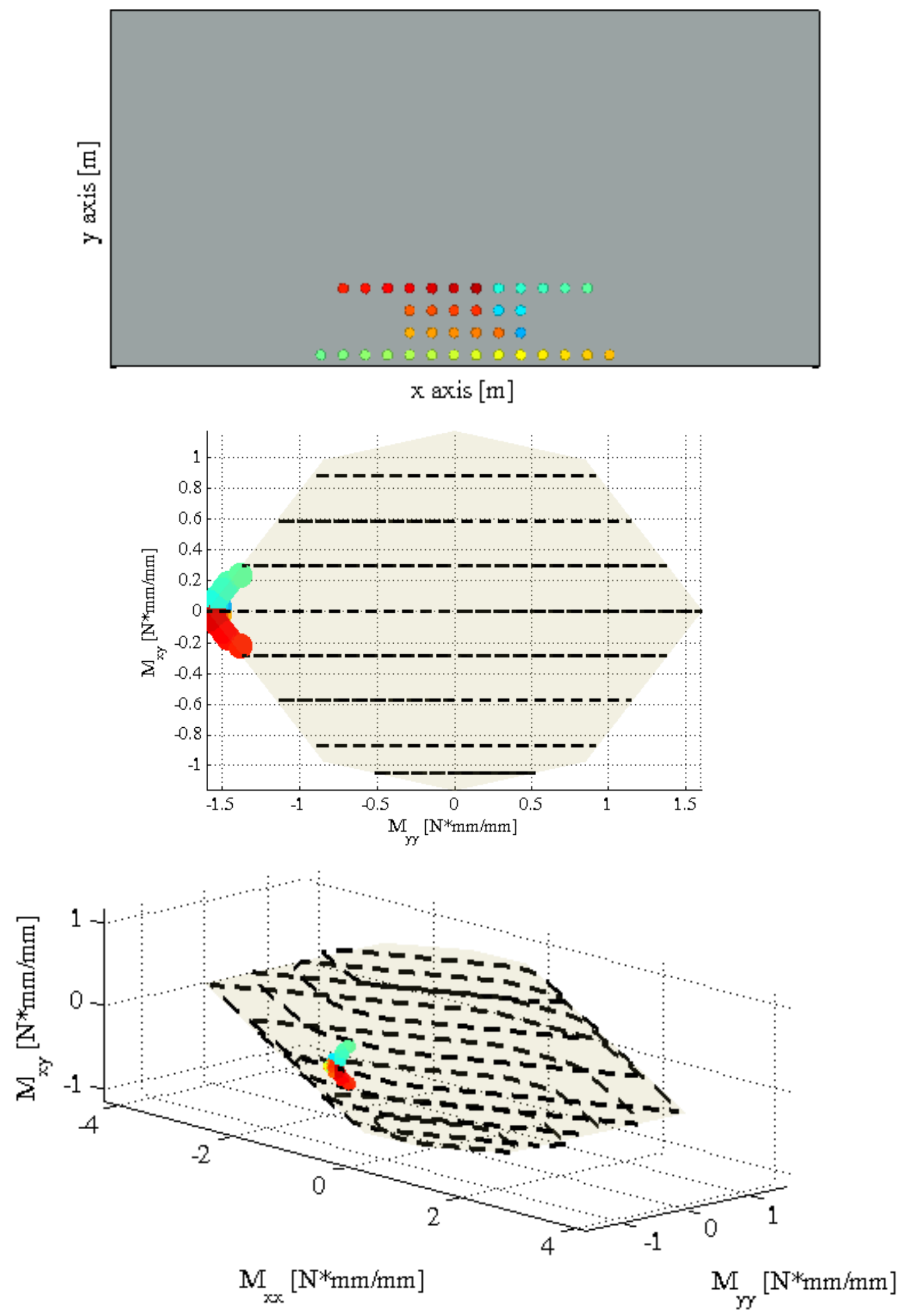

Figure 13: Panel SB01 reinforced through the stress-based optimization for $p=0.165 \mathrm{kN} / \mathrm{m}^{2}$. -a: Location of the elements where out-of-plane internal actions on masonry layer (bending moment and torque) stand on the failure surface (critical points). -b: Representation of the failure surface and the critical points on it. 


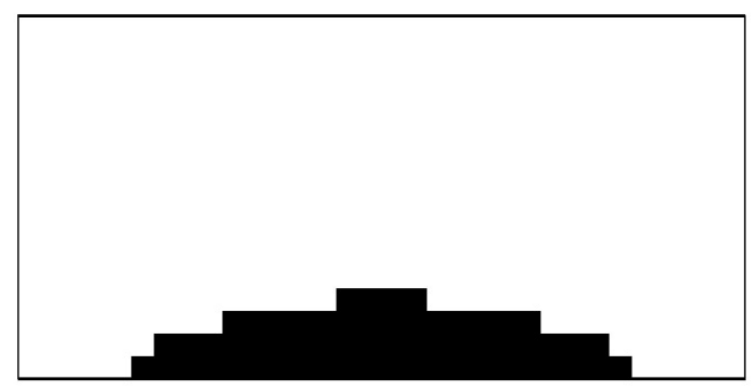

(a)

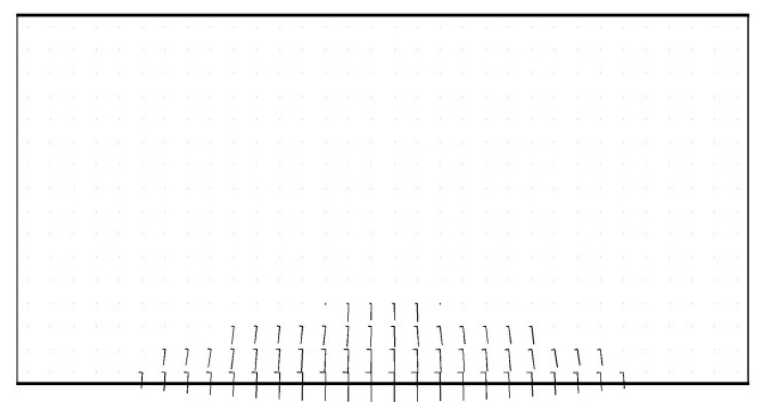

(b)

Figure 14: Panel SB01. Energy-based design for $p=0.165 \mathrm{kN} / \mathrm{m}^{2}$ and $V_{f}=0.25$. Optimal distribution of the fiber-reinforcement (a) and relevant principal stress directions (b), $w=1.35 \mathrm{~mm}$.

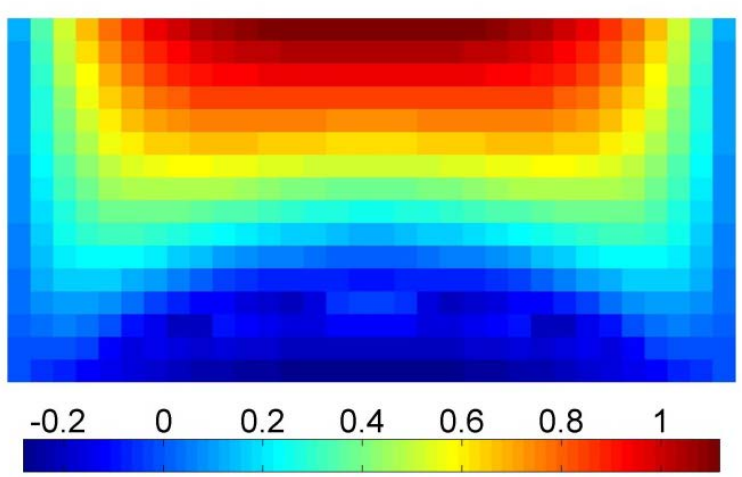

(a) $M_{x x}$

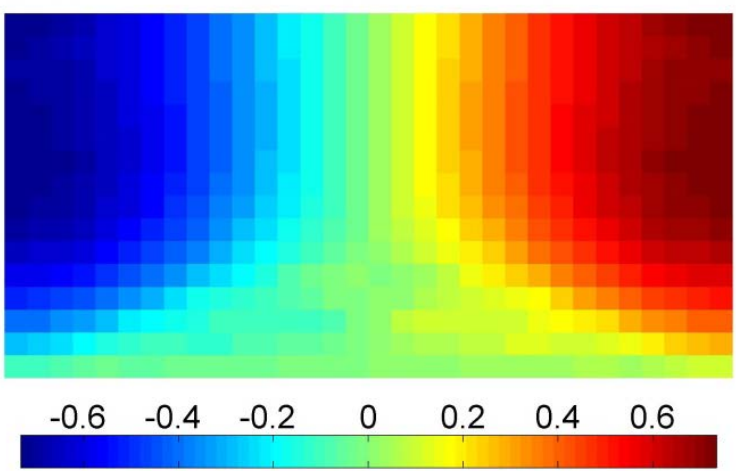

(c) $M_{x y}$

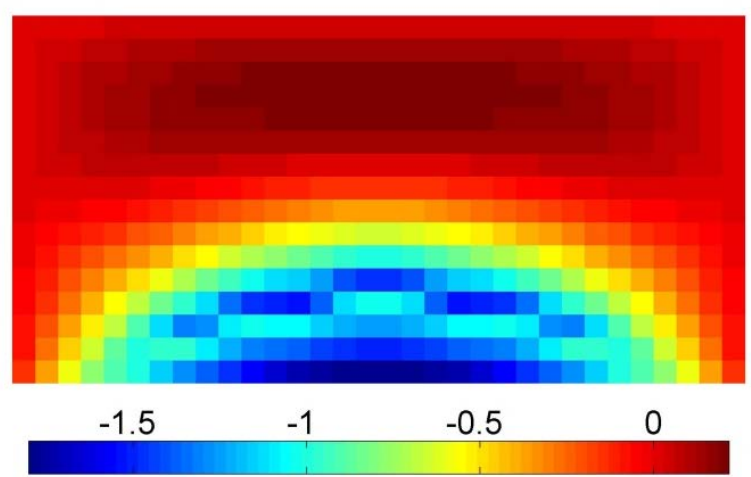

(b) $M_{y y}$

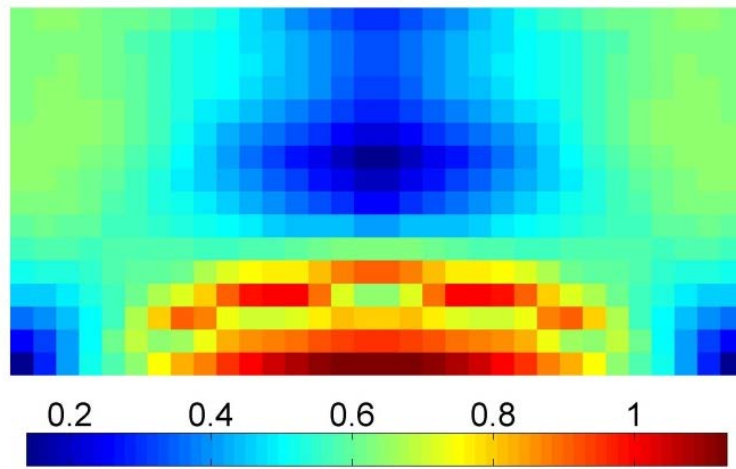

(d)

Figure 15. Panel SB01. Moments (in $\mathrm{kNm} / \mathrm{m}$ ) in the masonry layer of the panel reinforced through the energy-based optimization for $p=0.165 \mathrm{kN} / \mathrm{m}^{2}$ (a-c) and feasibility of the moment field with respect to the strength criterion (d). 


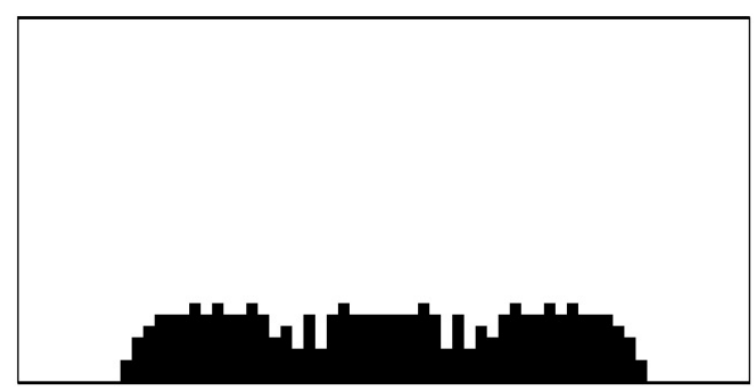

(a)

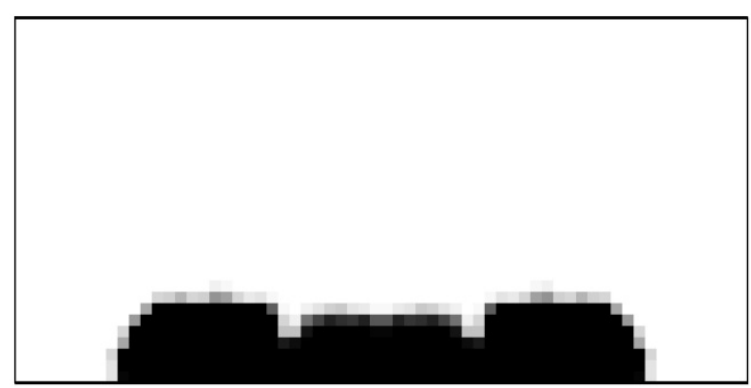

(b)

Figure 16: Panel SB01. Stress-based design for $p=0.160 \mathrm{kN} / \mathrm{m}^{2}$. Optimal distribution of the fiber-reinforcement over a mesh of 2024 elements with no filter (a) and with density filter (b). $V_{f}=0.25$. 


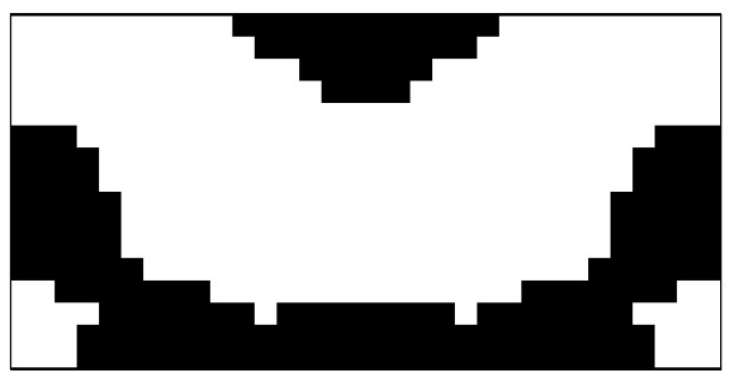

(a)

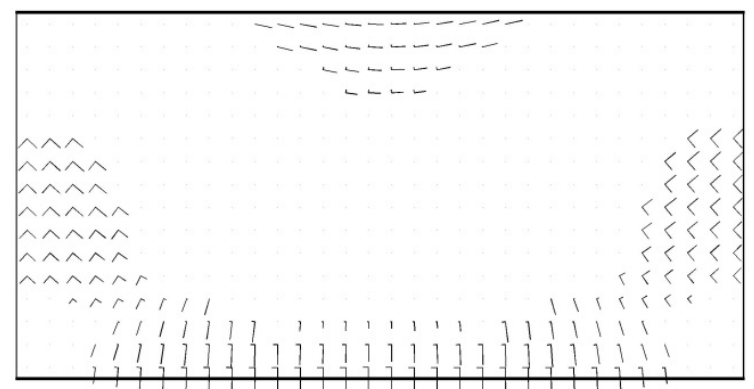

(b)

Figure 17: Panel SB01. Stress-based design for $p=0.185 \mathrm{kN} / \mathrm{m}^{2}$. Optimal distribution of the fiberreinforcement (a) and relevant principal stress directions (b), $V_{f}=0.70, w=1.32 \mathrm{~mm}$.

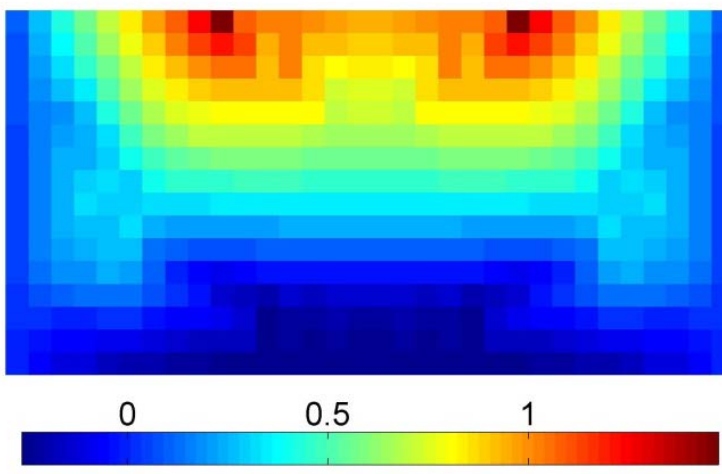

(a) $M_{x x}$

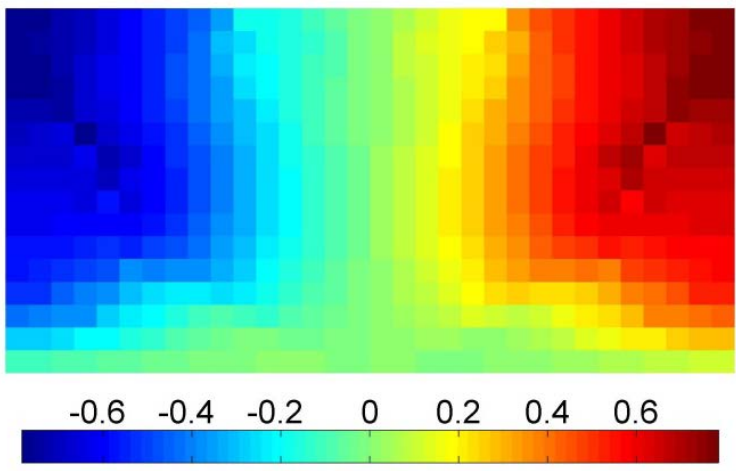

(c) $M_{x y}$

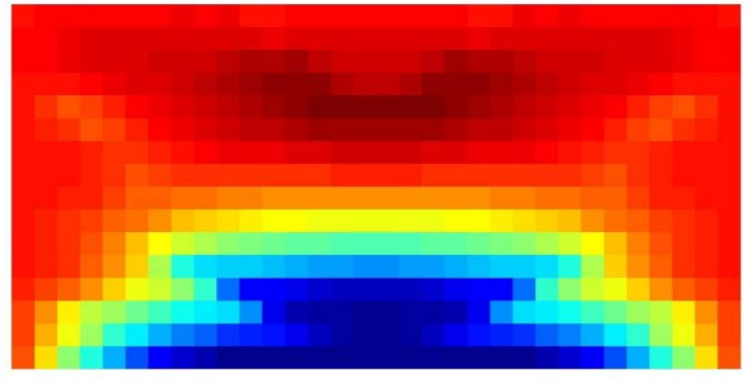

$\begin{array}{llll}-1.5 & -1 & -0.5 & 0\end{array}$

(b) $M_{y y}$

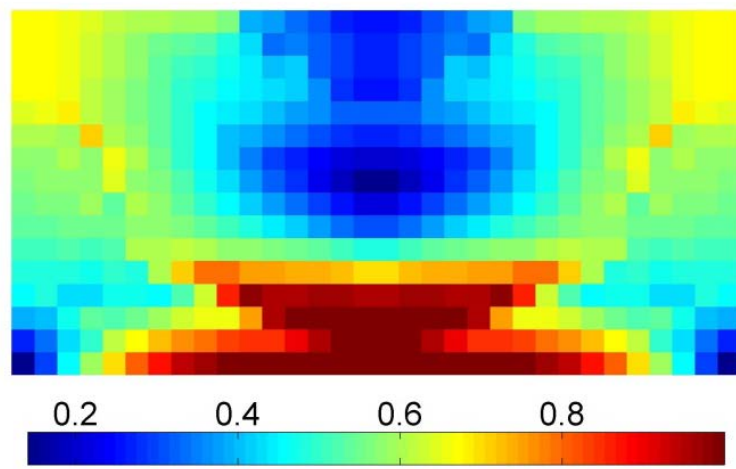

(d)

Figure 18: Moments (in $\mathrm{kNm} / \mathrm{m}$ ) in the masonry layer of the panel reinforced through the stress-based optimization for $p=0.185 \mathrm{kN} / \mathrm{m}^{2}$ (a-c) and feasibility of the moment field with respect to the strength criterion (d). 

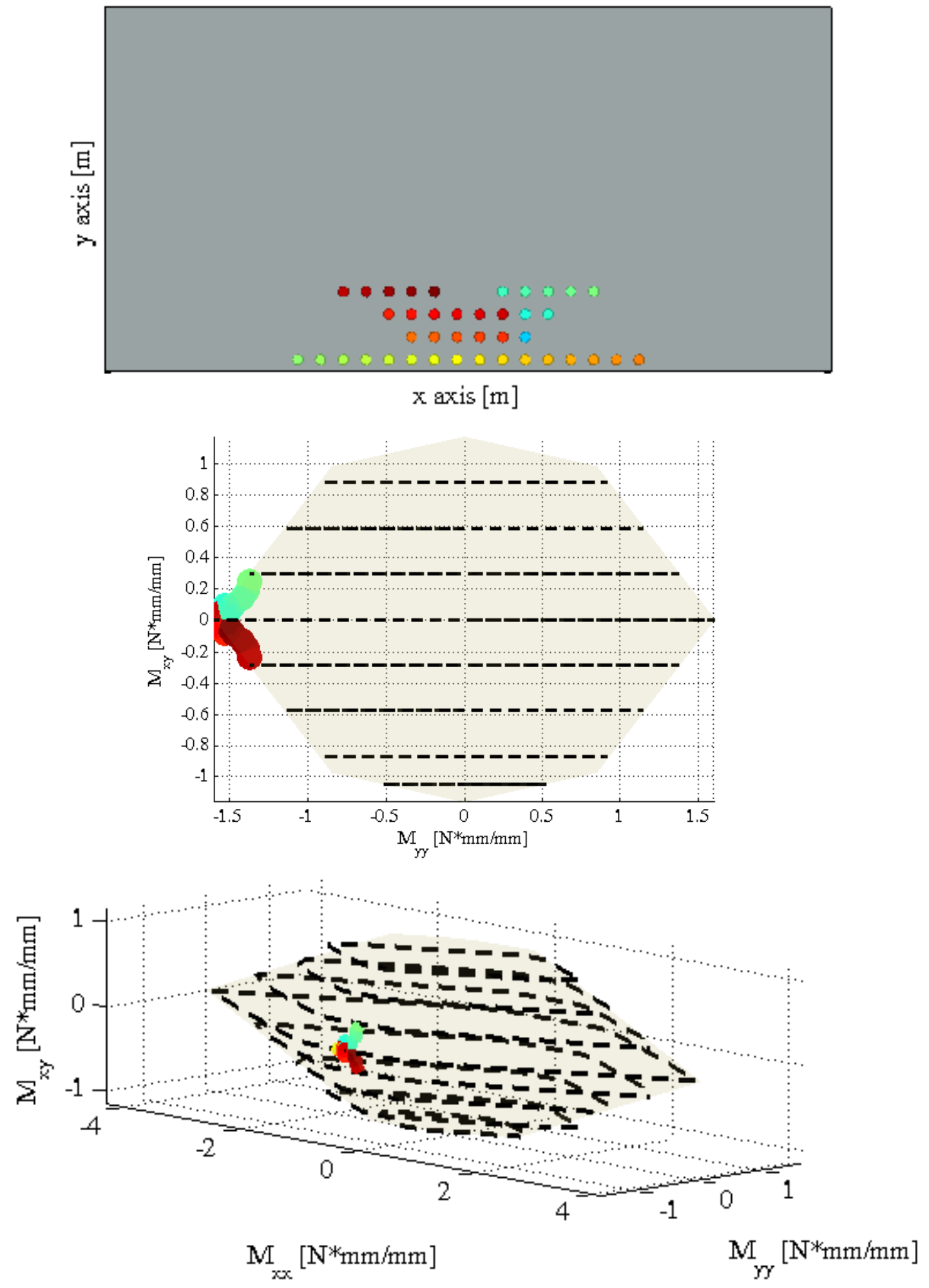

Figure 19: Panel SB01 reinforced through the stress-based optimization for $p=0.185 \mathrm{kN} / \mathrm{m}^{2}$. -a: Location of the elements where out-of-plane internal actions on masonry layer (bending moment and torque) stand on the failure surface (critical points). -b: Representation of the failure surface and the critical points on it. 


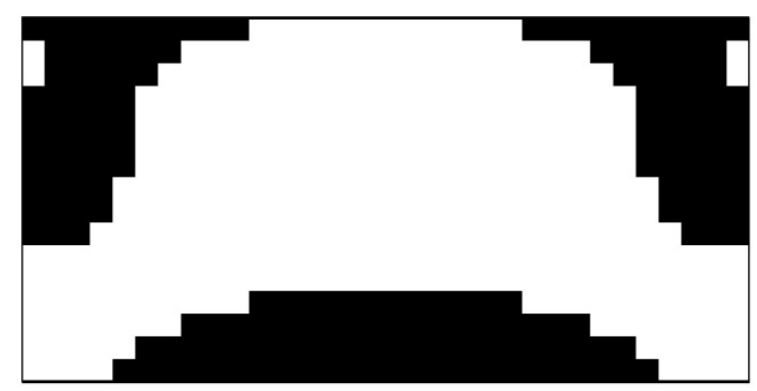

(a)

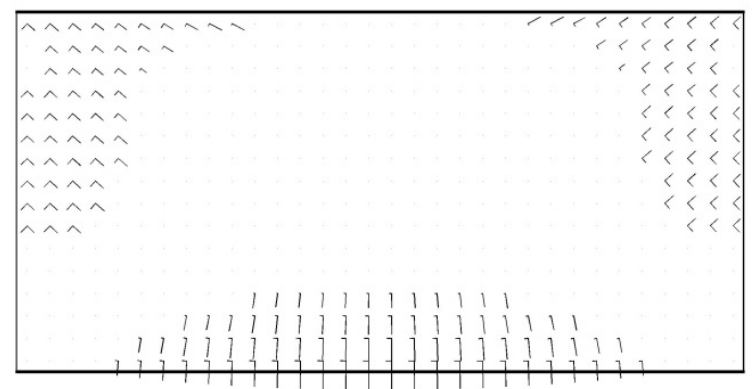

(b)

Figure 20: Panel SB01. Energy-based design for $p=0.185 \mathrm{kN} / \mathrm{m}^{2}$ and $V_{f}=0.70$. Optimal distribution of the fiber-reinforcement (a) and relevant principal stress directions (b), $w=1.27 \mathrm{~mm}$.

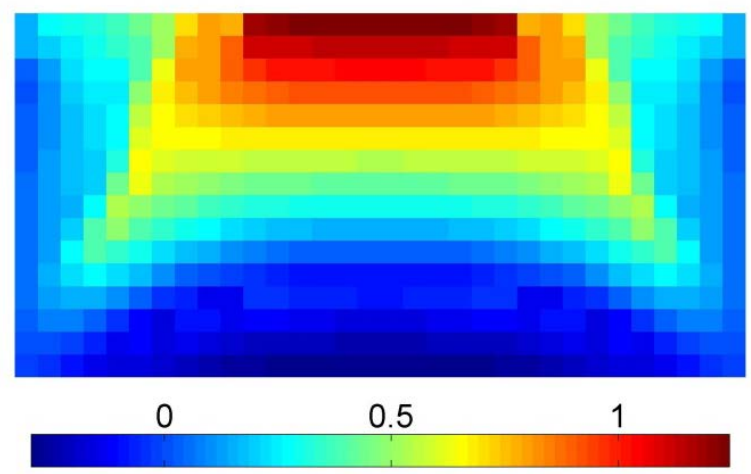

(a) $M_{X X}$

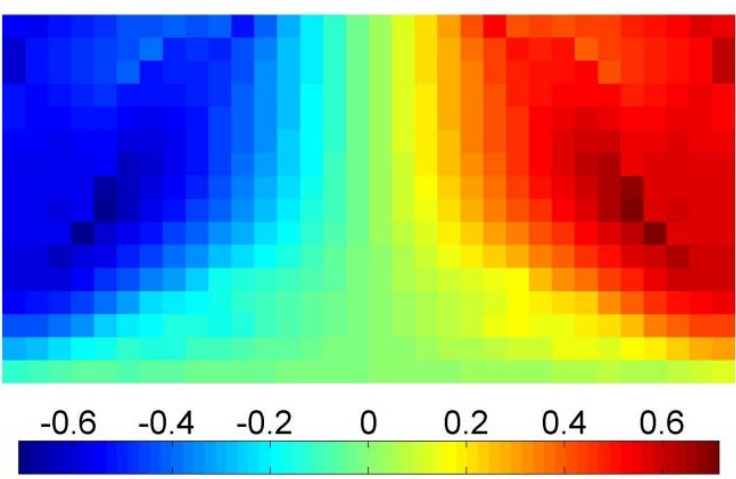

(c) $M_{x y}$

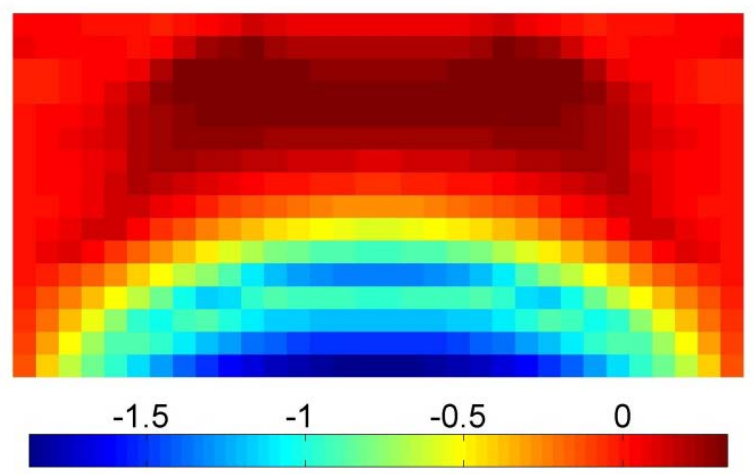

(b) $M_{y y}$

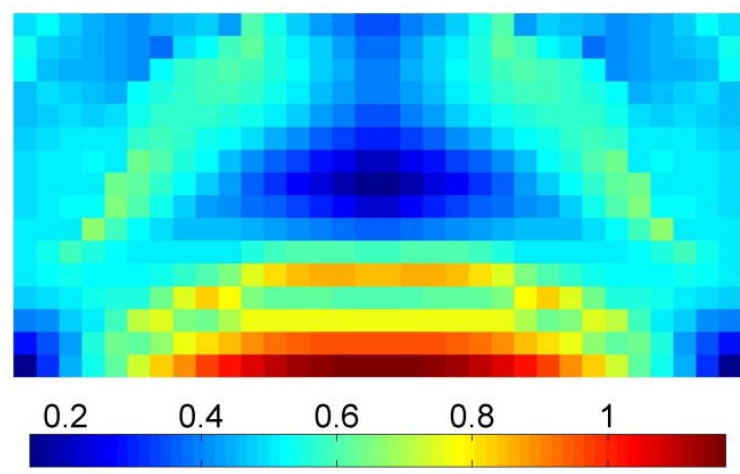

(d)

Figure 21: Panel SB01. Moments (in $\mathrm{kNm} / \mathrm{m}$ ) in the masonry layer of the panel reinforced through the energy-based optimization for $p=0.185 \mathrm{kN} / \mathrm{m}^{2}$ (a-c) and feasibility of the moment field with respect to the strength criterion (d). 

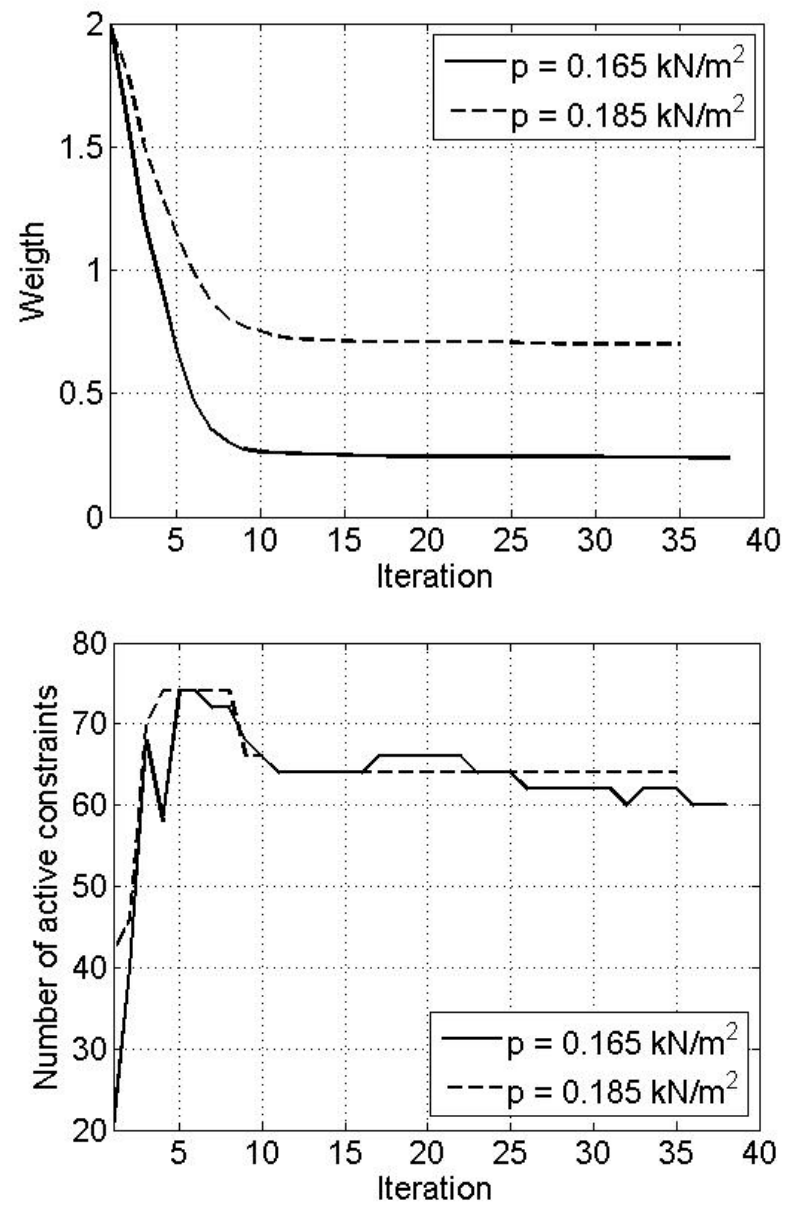

(a)

(b)

Figure 22: Reinforcement of Panel SB01 through the stress-based optimization: weight convergence (a) and number of active constraints (b) for $p=0.165 \mathrm{kN} / \mathrm{m}^{2}$ and $p=0.185 \mathrm{kN} / \mathrm{m}^{2}$.

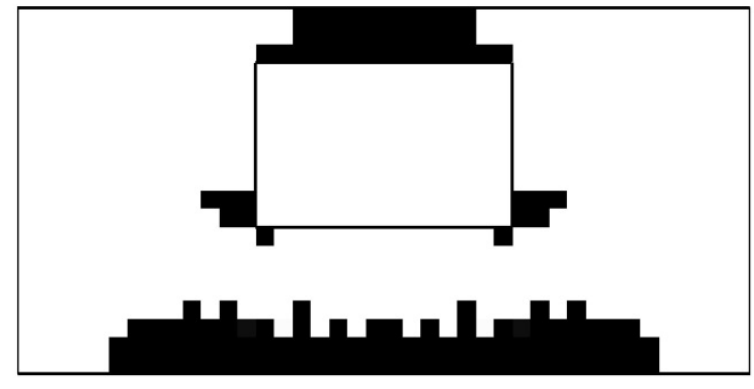

(a)

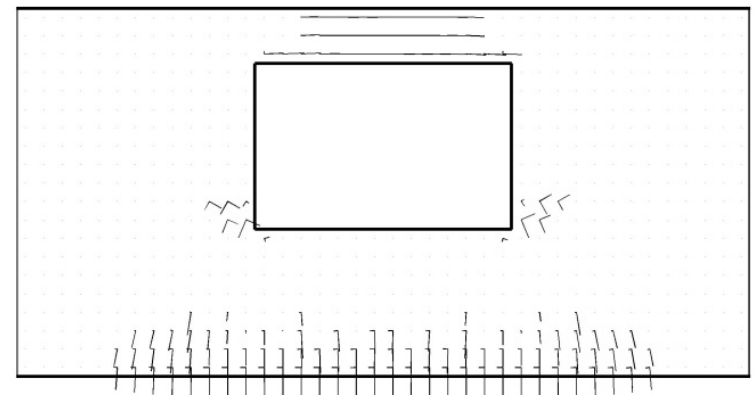

(b)

Figure 23: Panel SB02. Stress-based design for $p=0.250 \mathrm{kN} / \mathrm{m}^{2}$. Optimal distribution of the fiberreinforcement (a) and relevant principal stress directions (b), $V_{f}=0.40, w=1.72 \mathrm{~mm}$. 
CAS-D-14-00307 Revised version, modifications highlighted with color YELLOW 


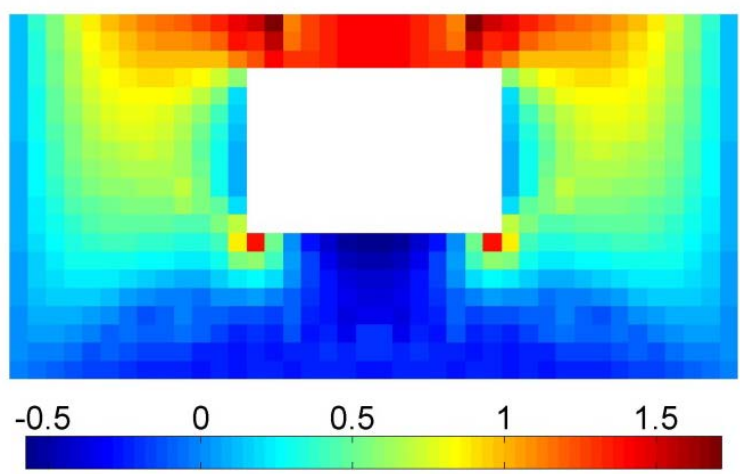

(a) $M_{x x}$

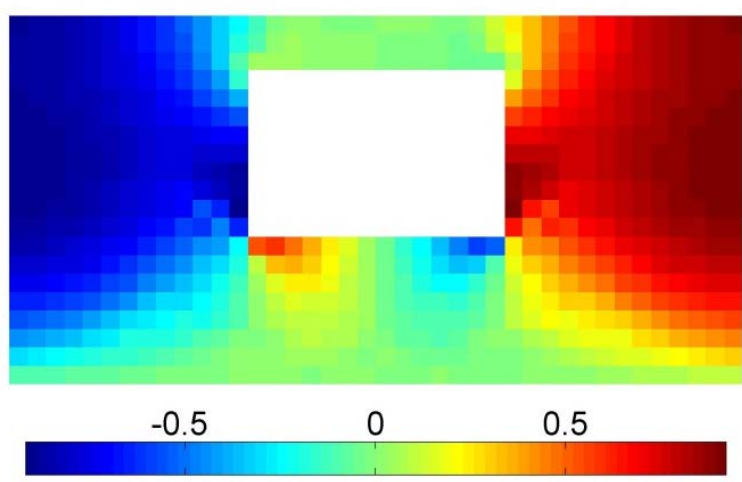

(c) $M_{x y}$

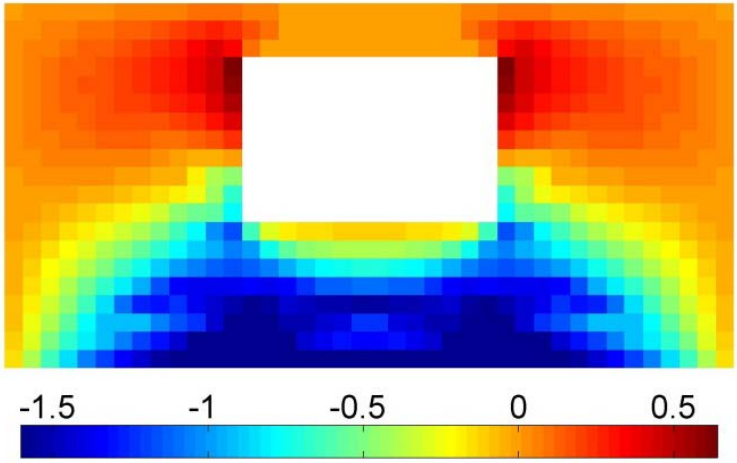

(b) $M_{y y}$

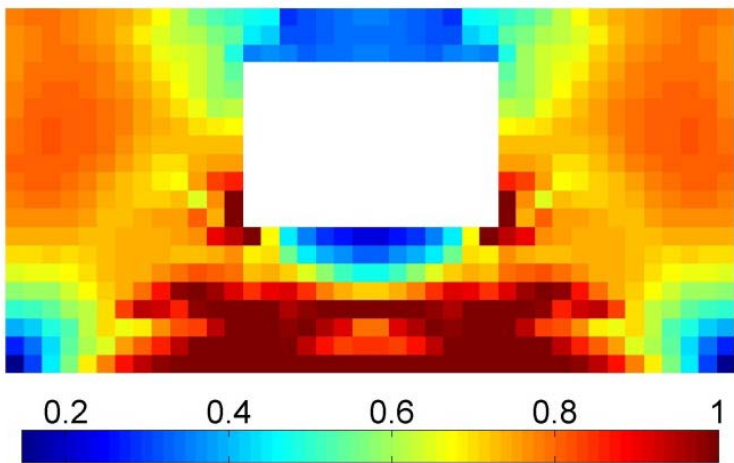

(d)

Figure 24: Panel SB02. Moments (in $\mathrm{kNm} / \mathrm{m}$ ) in the masonry layer of the panel reinforced through the stress-based optimization for $p=0.250 \mathrm{kN} / \mathrm{m}^{2}$ (a-c) and feasibility of the moment field with respect to the strength criterion (d). 

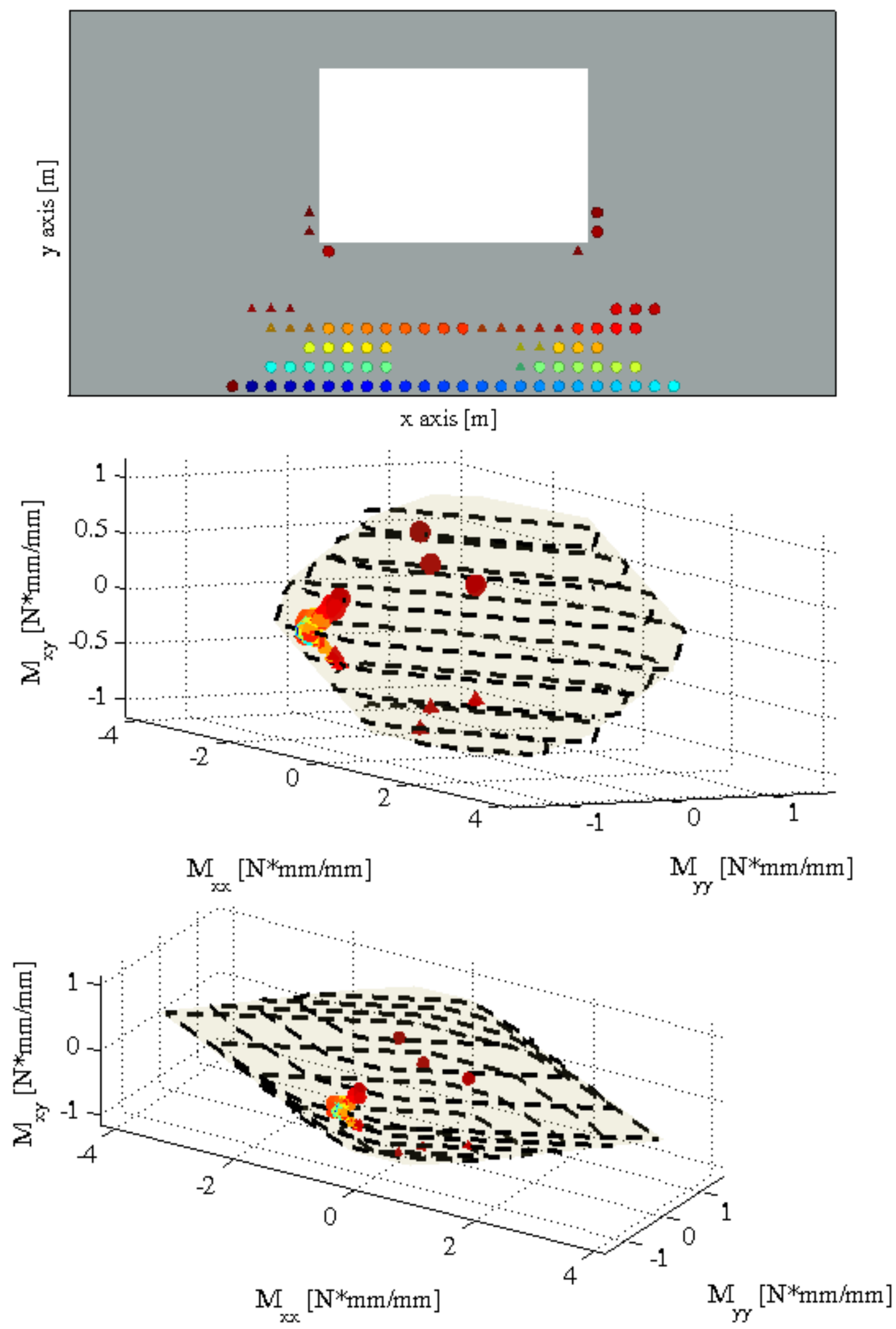

Figure 25: Panel SB02 reinforced through the stress-based optimization for $p=0.250 \mathrm{kN} / \mathrm{m}^{2}$. -a: Location of the elements where out-of-plane internal actions on masonry layer (bending moment and torque) stand on the failure surface (critical points). -b: Representation of the failure surface and the critical points on it. 
CAS-D-14-00307 Revised version, modifications highlighted with color YELLOW 


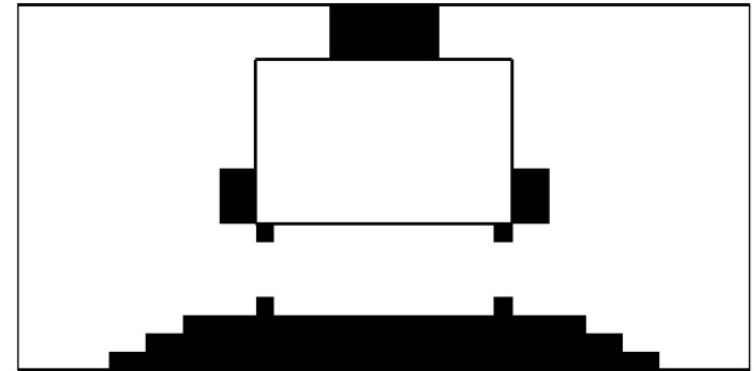

(a)

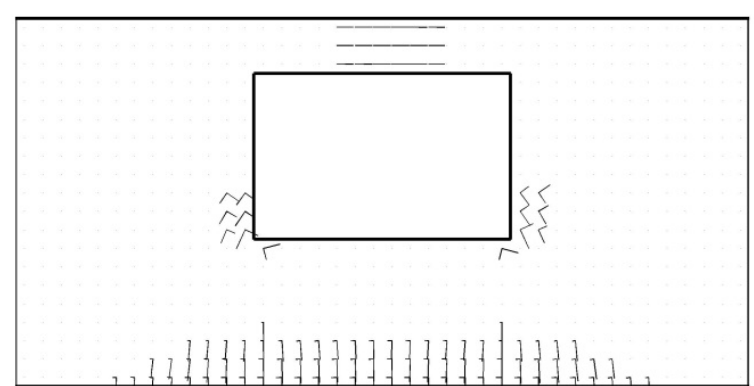

(b)

Figure 26: Panel SB02. Energy-based design for $p=0.250 \mathrm{kN} / \mathrm{m}^{2}$ and $V_{f}=0.40$. Optimal distribution of the fiber-reinforcement (a) and relevant principal stress directions (b), $w=1.70 \mathrm{~mm}$.

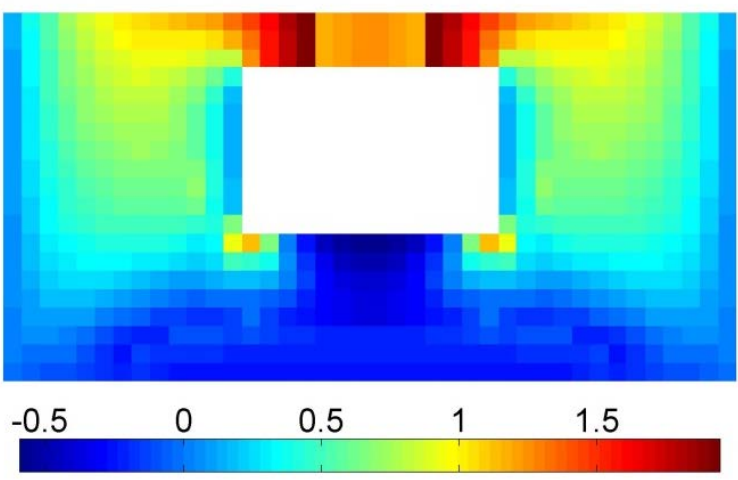

(a) $M_{x x}$

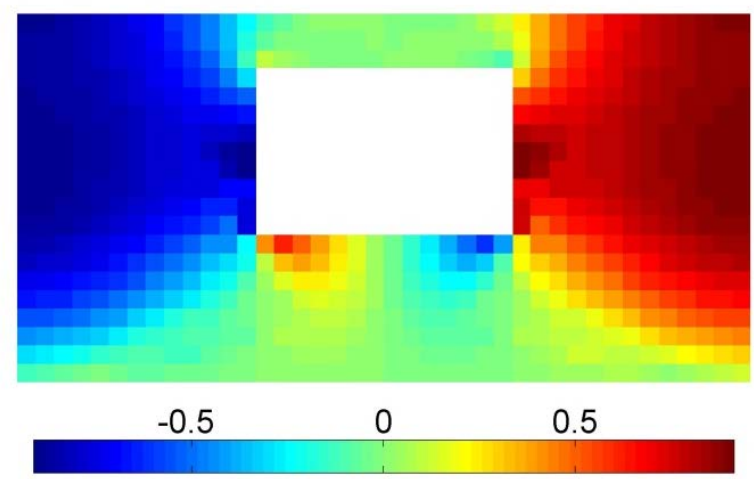

(c) $M_{x y}$

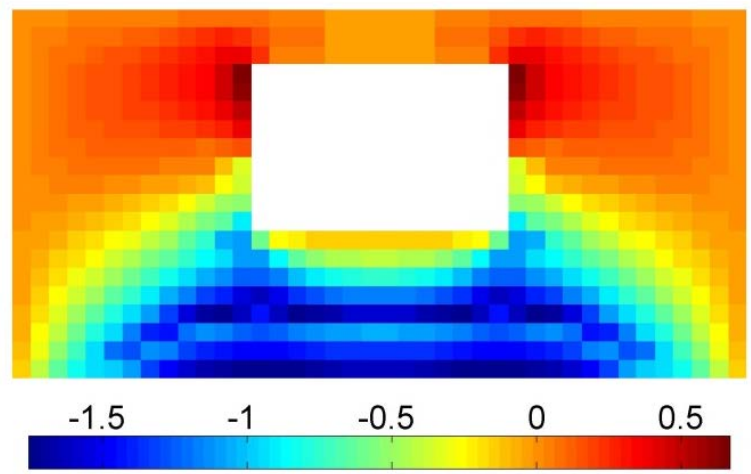

(b) $M_{y y}$

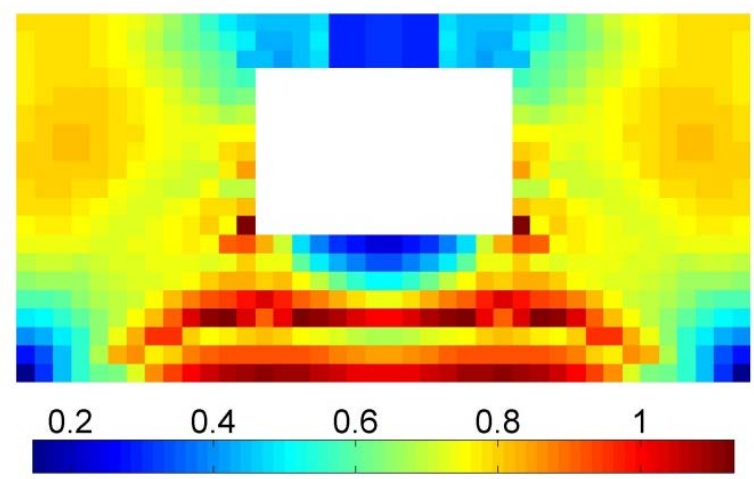

(d)

Figure 27: Panel SB02. Moments (in $\mathrm{kNm} / \mathrm{m}$ ) in the masonry layer of the panel reinforced through the energy-based optimization for $p=0.250 \mathrm{kN} / \mathrm{m}^{2}$ (a-c) and feasibility of the moment field with respect to the strength criterion (d). 
CAS-D-14-00307 Revised version, modifications highlighted with color YELLOW

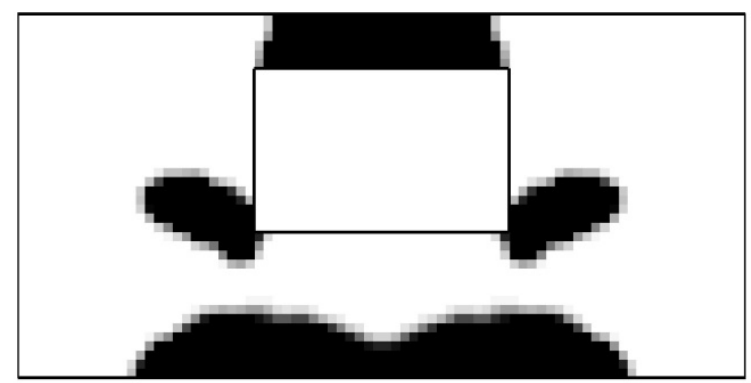

(a)

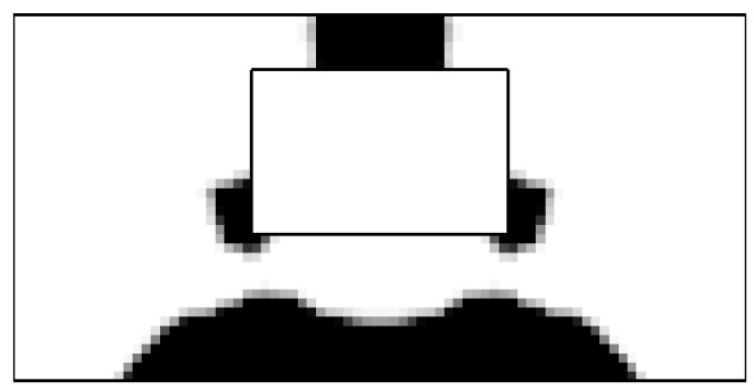

(b)

Figure 28. Panel SB02. Stress-based design for $p=0.225 \mathrm{kN} / \mathrm{m}^{2}\left(V_{f}=0.40\right.$ ) (a) and energy-based design for $p=0.225 \mathrm{kN} / \mathrm{m}^{2}$ and $V_{f}=0.40(\mathrm{~b})$, mesh of 2696 elements. 


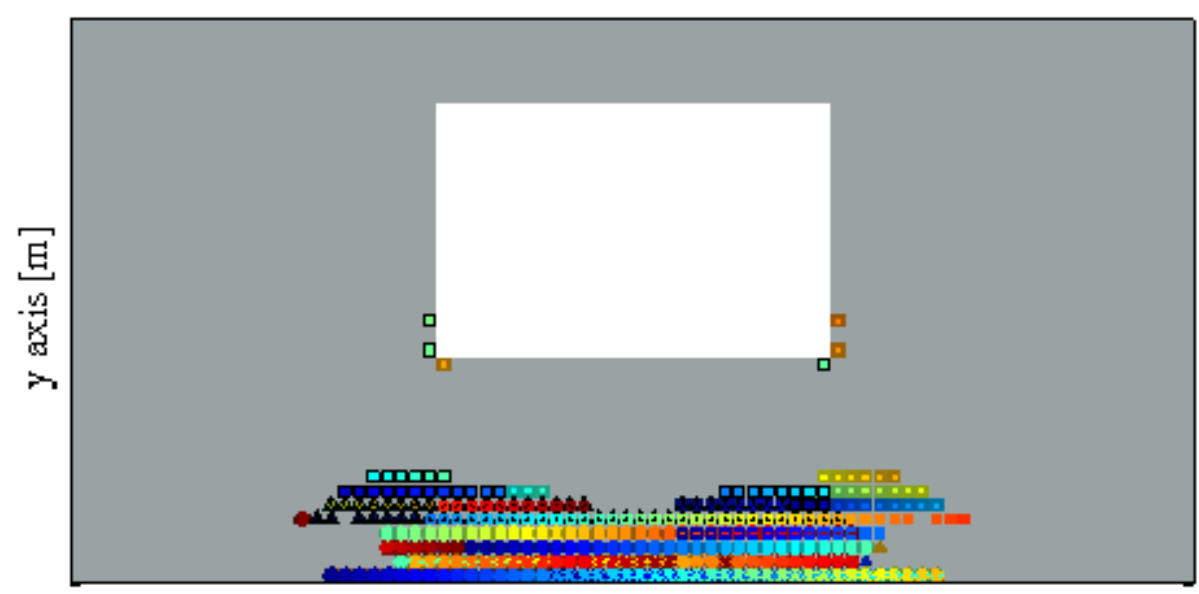

$$
\mathrm{x} \text { axis }[\mathrm{m}]
$$
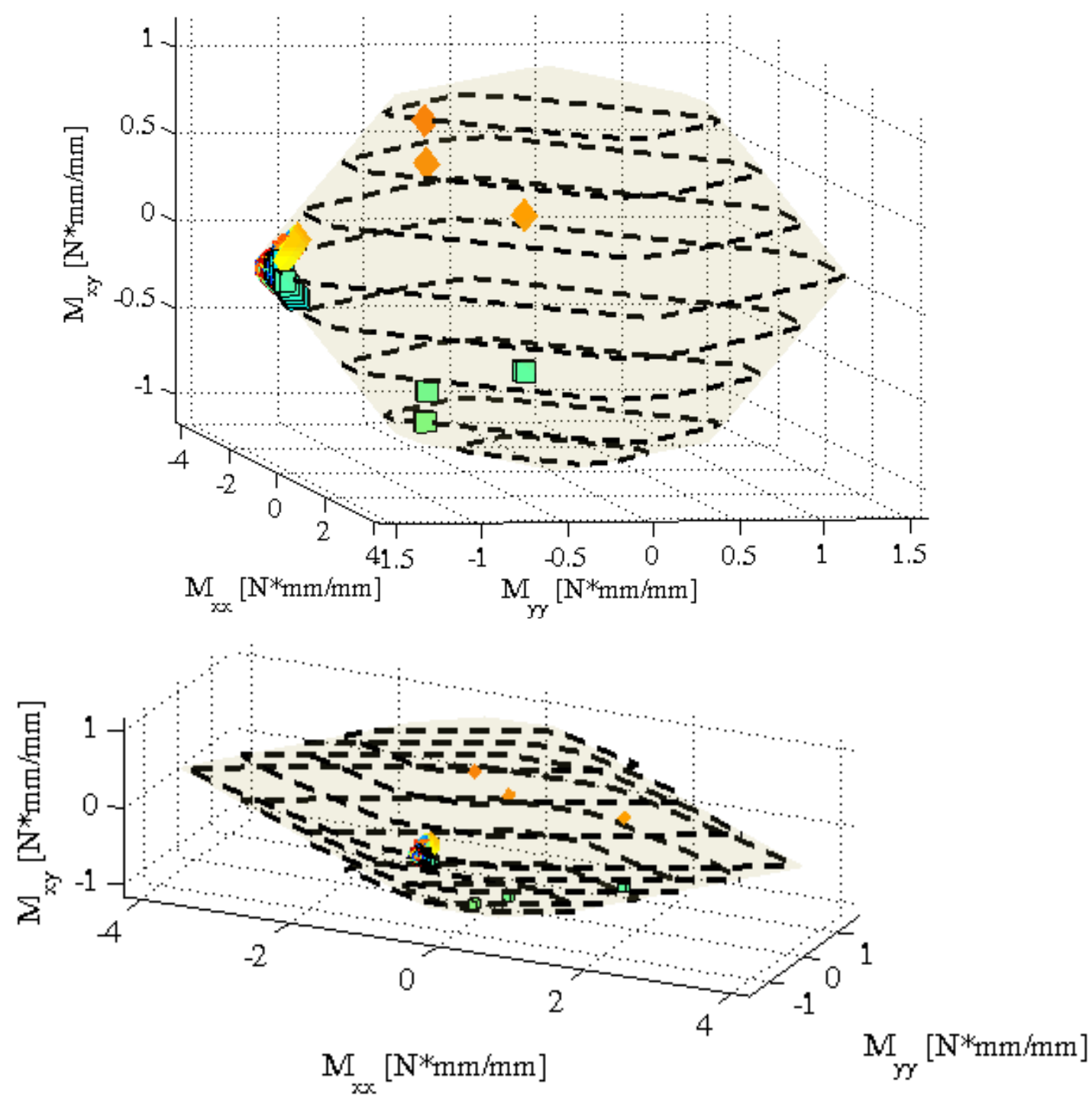

Figure 29: Panel SB02 reinforced through the stress-based optimization for $p=0.225 \mathrm{kN} / \mathrm{m}^{2}$. -a: Location of the elements where out-of-plane internal actions on masonry layer (bending moment and torque) stand on the failure surface (critical points). -b: Representation of the failure surface and the critical points on it. 


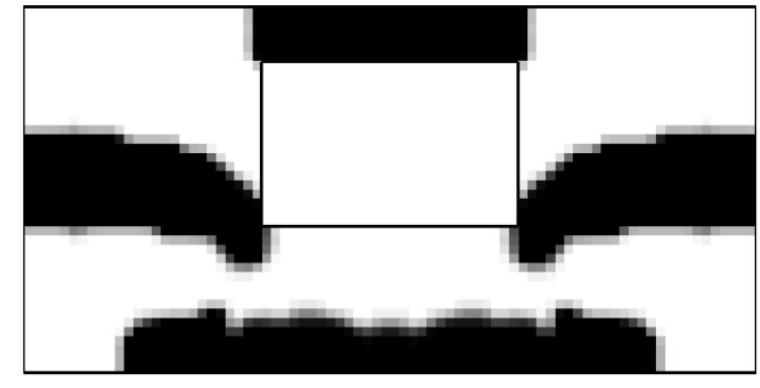

(a)

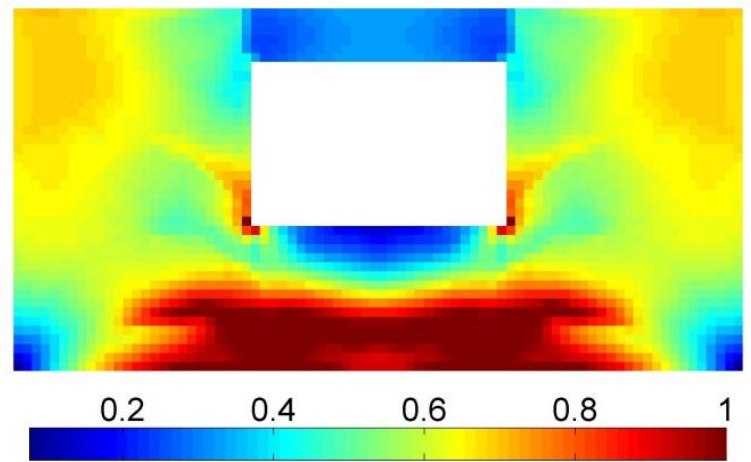

(b)

Figure 30: Panel SB02. Stress-based design for $p=0.240 \mathrm{kN} / \mathrm{m}^{2}$. Optimal distribution of the fiber-reinforcement over a mesh of 2696 elements (a) and feasibility of the moment field with respect to the strength criterion (b) $V_{f}=0.70, w=1.51 \mathrm{~mm}$. 


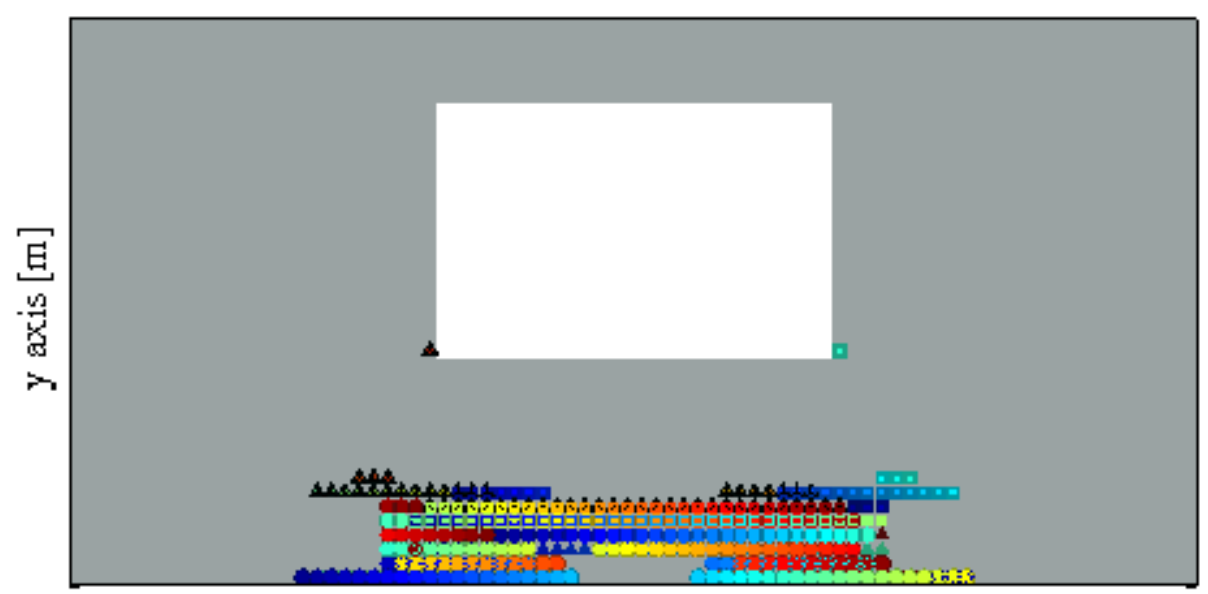

$\mathrm{x}$ axis $[\mathrm{m}]$
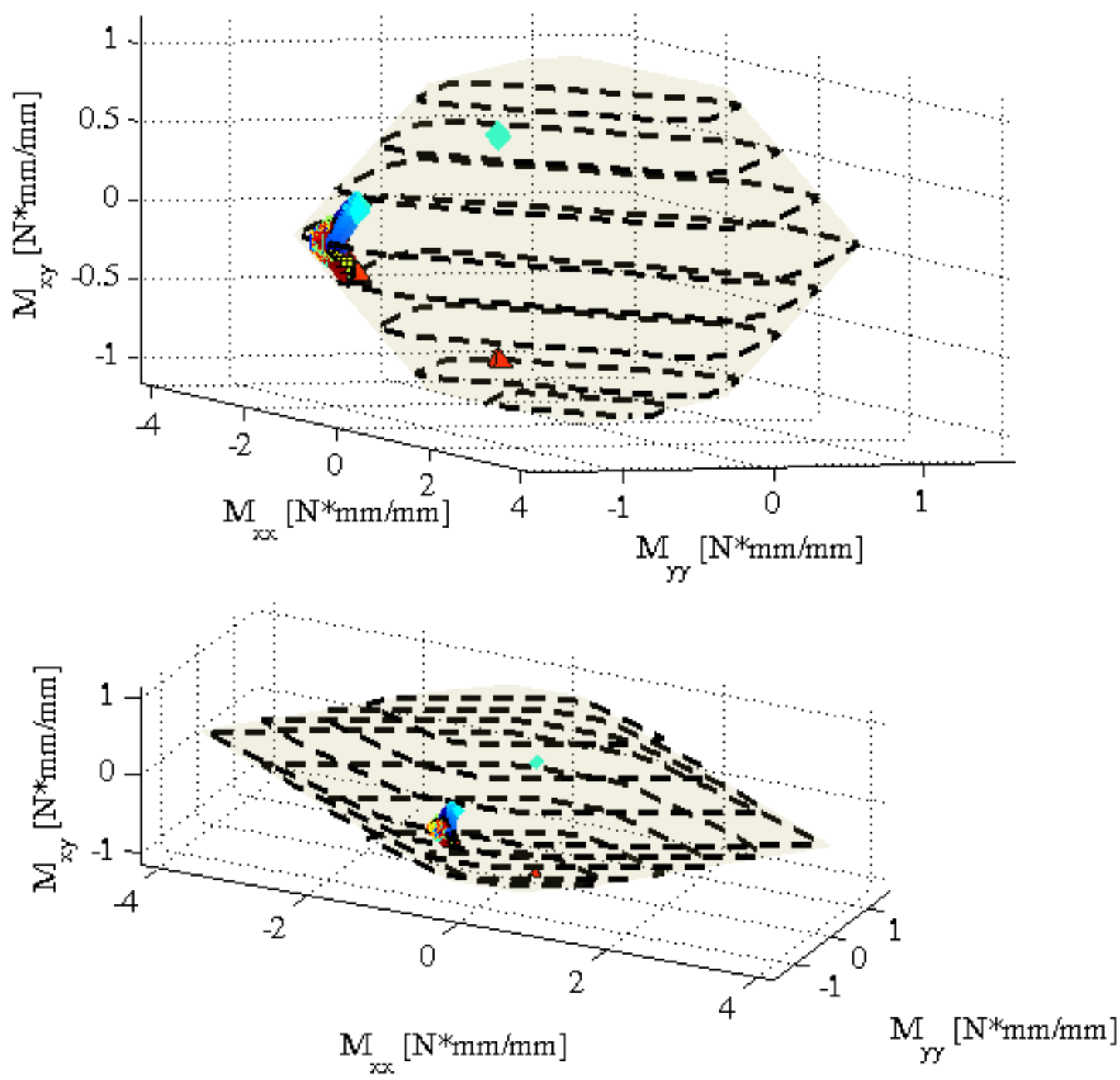

Figure 31: Panel SB02 reinforced through the stress-based optimization for $p=0.240 \mathrm{kN} / \mathrm{m}^{2}$. -a: Location of the elements where out-of-plane internal actions on masonry layer (bending moment and torque) stand on the failure surface (critical points). -b: Representation of the failure surface and the critical points on it. 


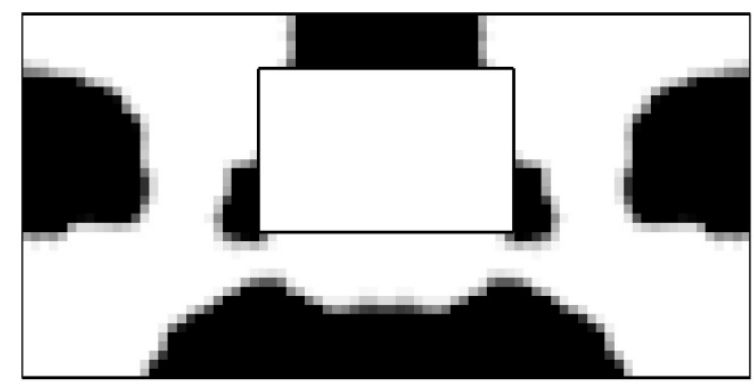

(a)

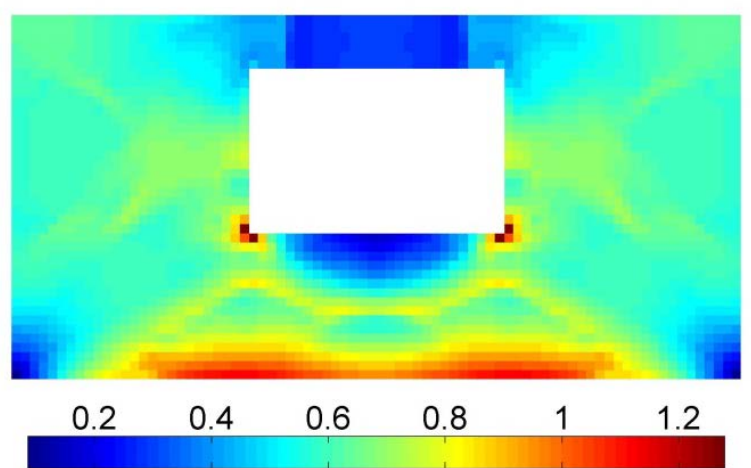

(b)

Figure 32: Panel SB02. Energy-based design for $p=0.240 \mathrm{kN} / \mathrm{m}^{2}$ and $V_{f}=0.70$. Optimal distribution of the fiber-reinforcement over a mesh of 2696 elements (a) and feasibility of the moment field with respect to the strength criterion (b), $w=1.46 \mathrm{~mm}$. 


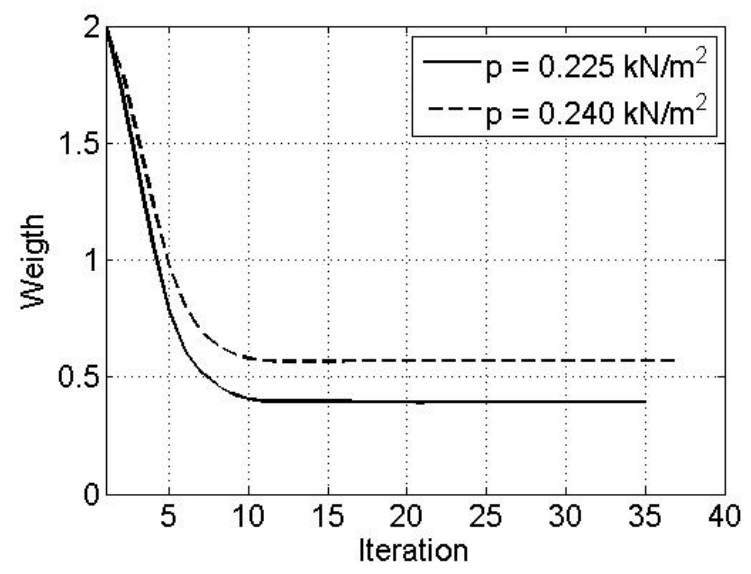

(a)

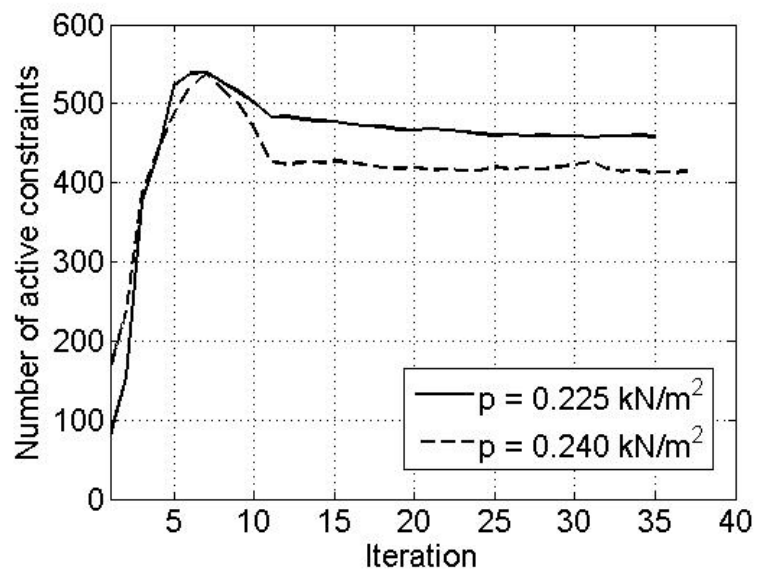

(b)

Figure 33. Reinforcement of Panel SB02 through the stress-based optimization: weight convergence (a) and number of active constraints (b) for $p=0.225 \mathrm{kN} / \mathrm{m}^{2}$ and $p=0.240 \mathrm{kN} / \mathrm{m}^{2}$ over a mesh of 2696 elements. 
CAS-D-14-00307 Revised version, modifications highlighted with color YELLOW

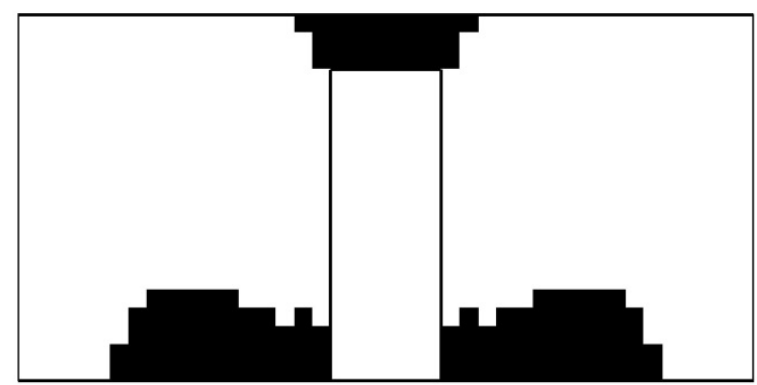

(a)

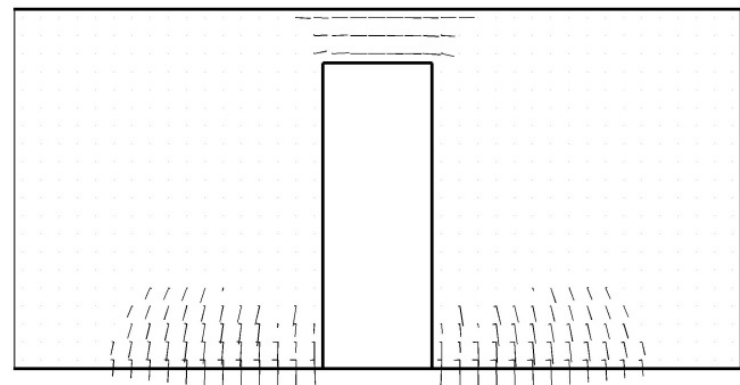

(b)

Figure 34: Panel SB03. Stress-based design for $p=0.155 \mathrm{kN} / \mathrm{m}^{2}$. Optimal distribution of the fiberreinforcement (a) and relevant principal stress directions (b), $V_{f}=0.35, w=1.26 \mathrm{~mm}$. 


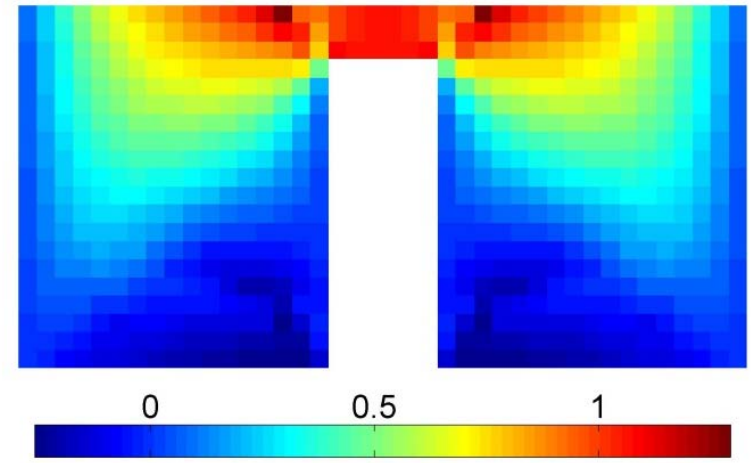

(a) $M_{x x}$

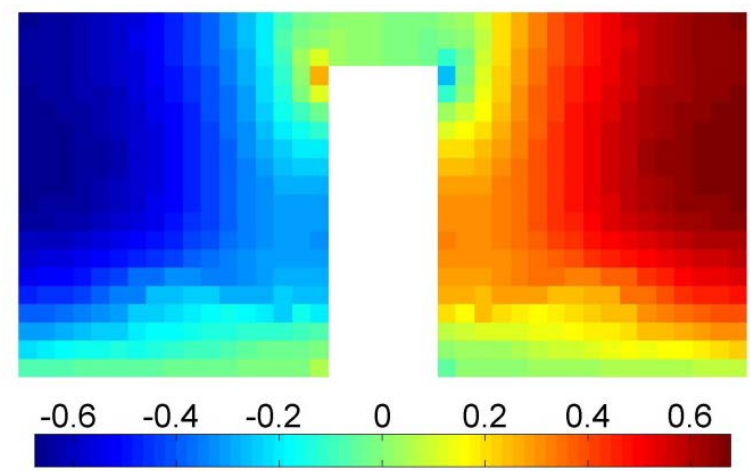

(c) $M_{x y}$

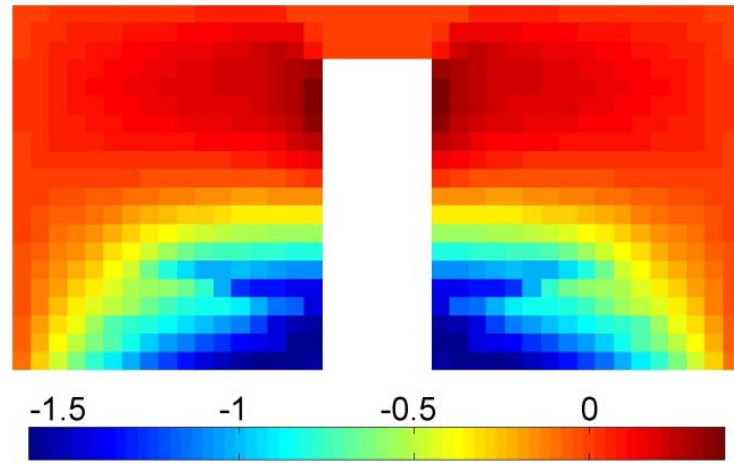

(b) $M_{y y}$

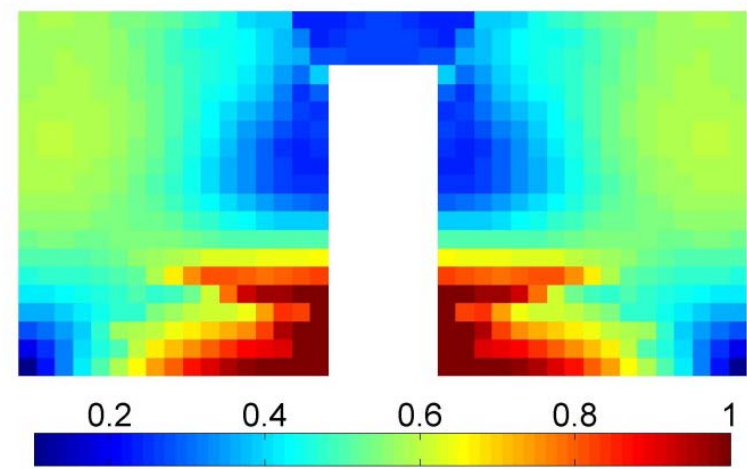

(d)

Figure 35: Panel SB03. Moments (in $\mathrm{kNm} / \mathrm{m}$ ) in the masonry layer of the panel reinforced through the stress-based optimization for $p=0.155 \mathrm{kN} / \mathrm{m}^{2}$ (a-c) and feasibility of the moment field with respect to the strength criterion (d).

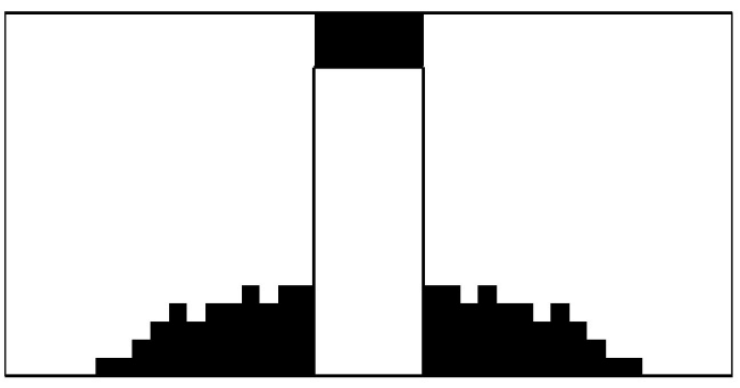

(a)

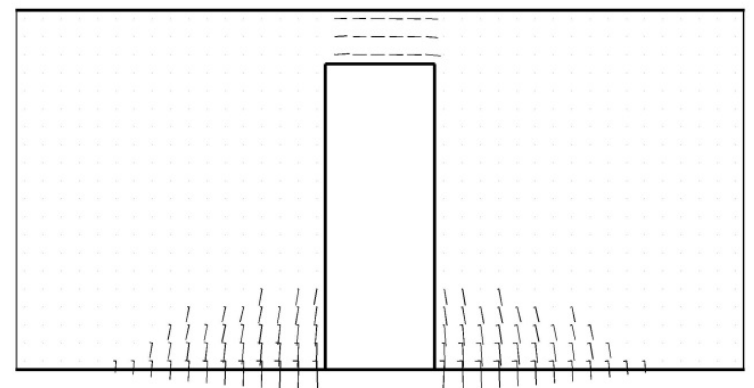

(b)

Figure 36: Panel SB03. Energy-based design for $p=0.155 \mathrm{kN} / \mathrm{m}^{2}$ and $V_{f}=0.35$. Optimal distribution of the fiber-reinforcement (a) and relevant principal stress directions (b), $w=1.24 \mathrm{~mm}$. 


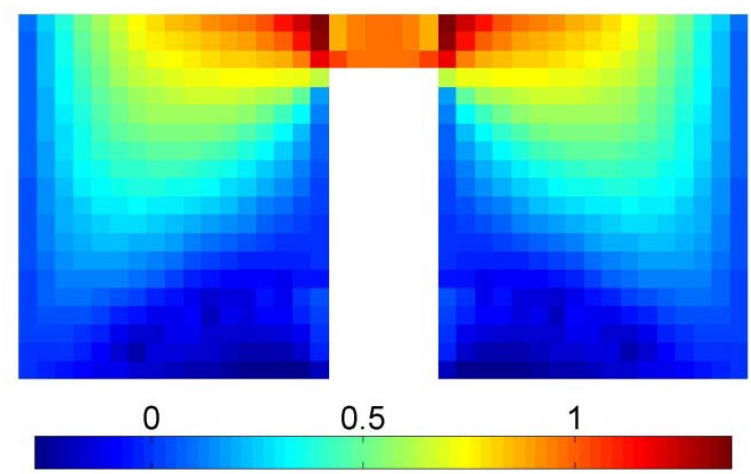

(a) $M_{x x}$

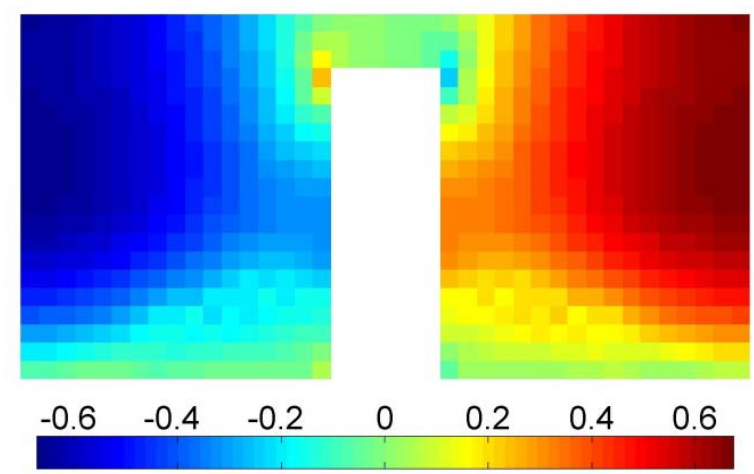

(c) $M_{x y}$

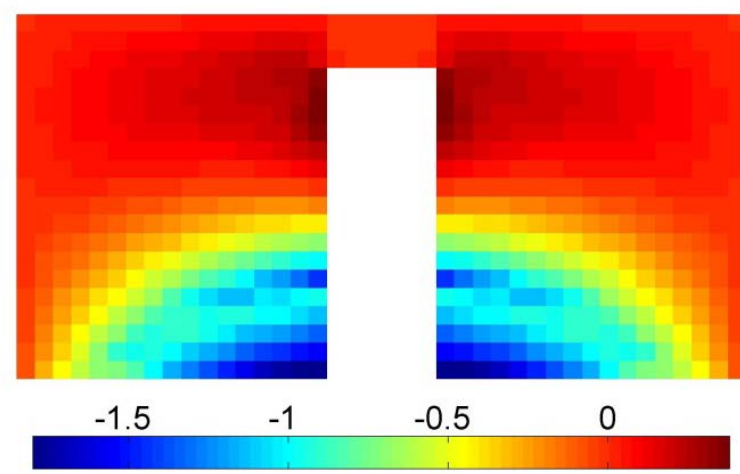

(b) $M_{y y}$

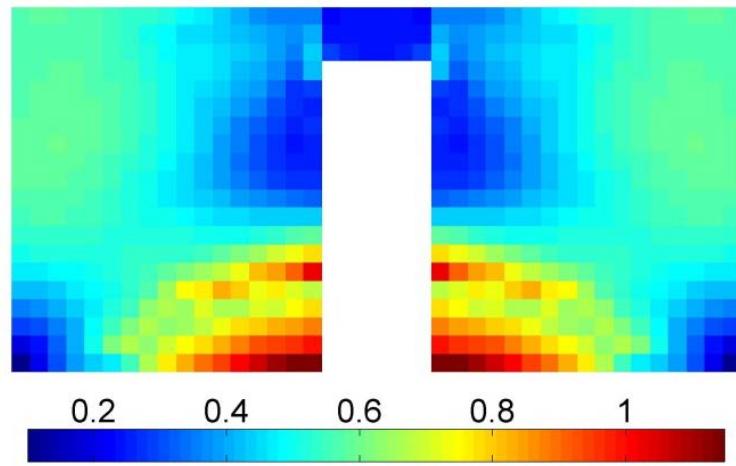

(d)

Figure 37: Panel SB03. Moments (in $\mathrm{kNm} / \mathrm{m}$ ) in the masonry layer of the panel reinforced through the energy-based optimization for $p=0.155 \mathrm{kN} / \mathrm{m}^{2}$ (a-c) and feasibility of the moment field with respect to the strength criterion (d). 

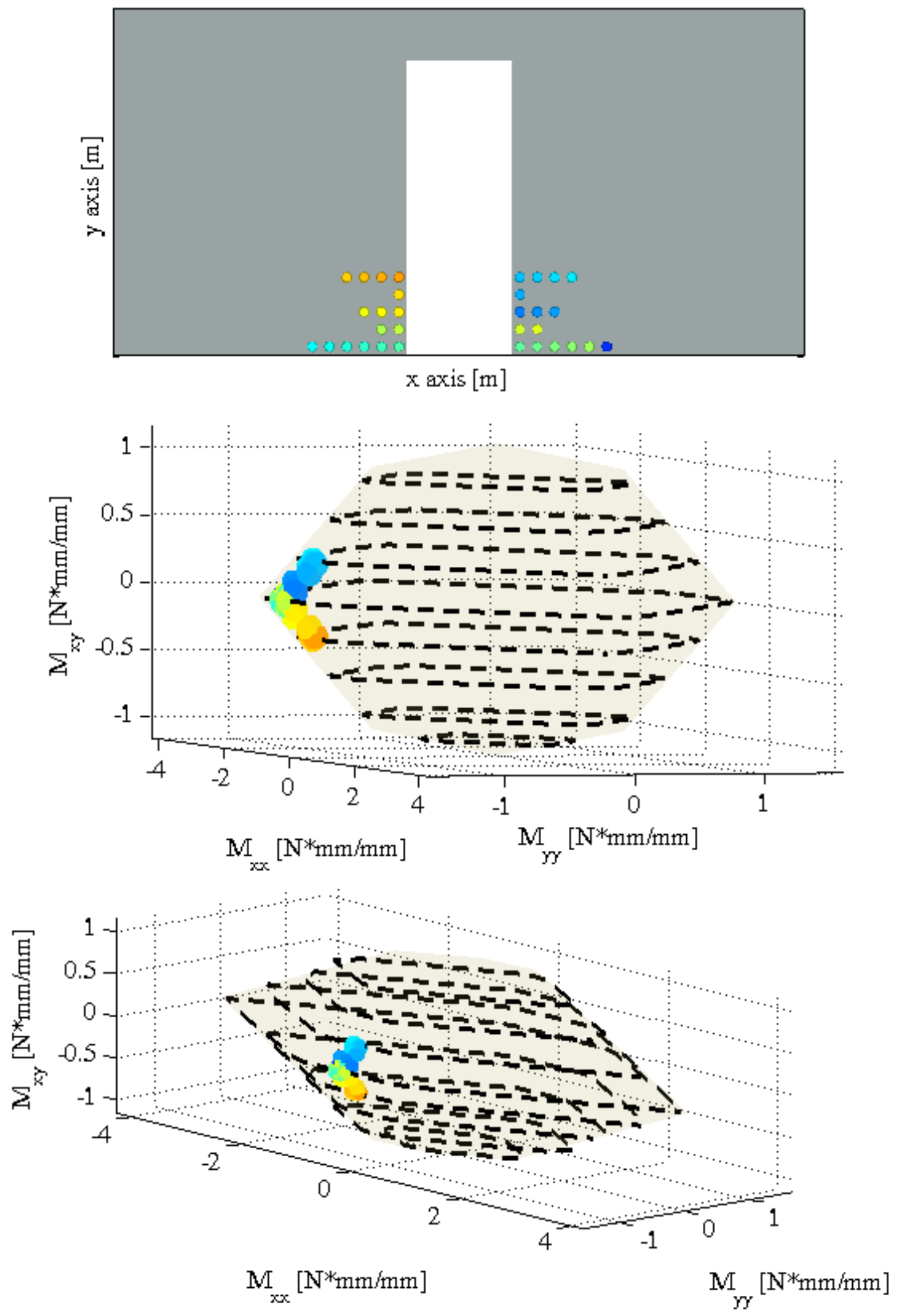

Figure 38: Panel SB03 reinforced through the stress-based optimization for $p=0.155 \mathrm{kN} / \mathrm{m}^{2}$.

Failure surface with 24 planes. -a: Location of the elements where out-of-plane internal actions on masonry layer (bending moment and torque) stand on the failure surface (critical points). -b: Representation of the failure surface and the critical points on it. 


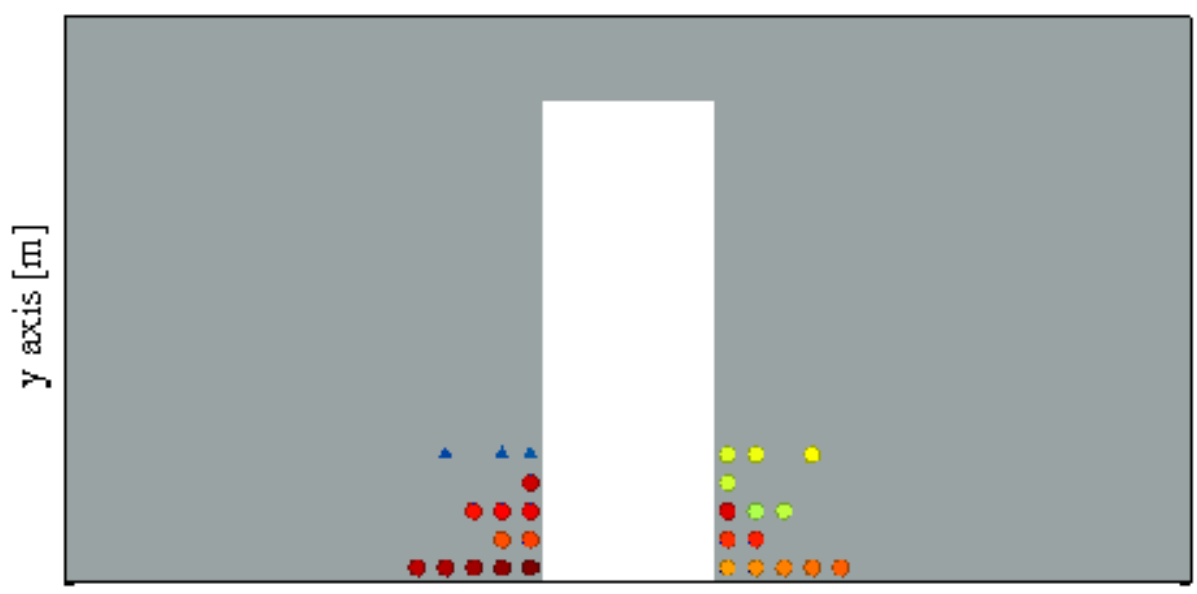

$\mathrm{x}$ axis $[\mathrm{m}]$
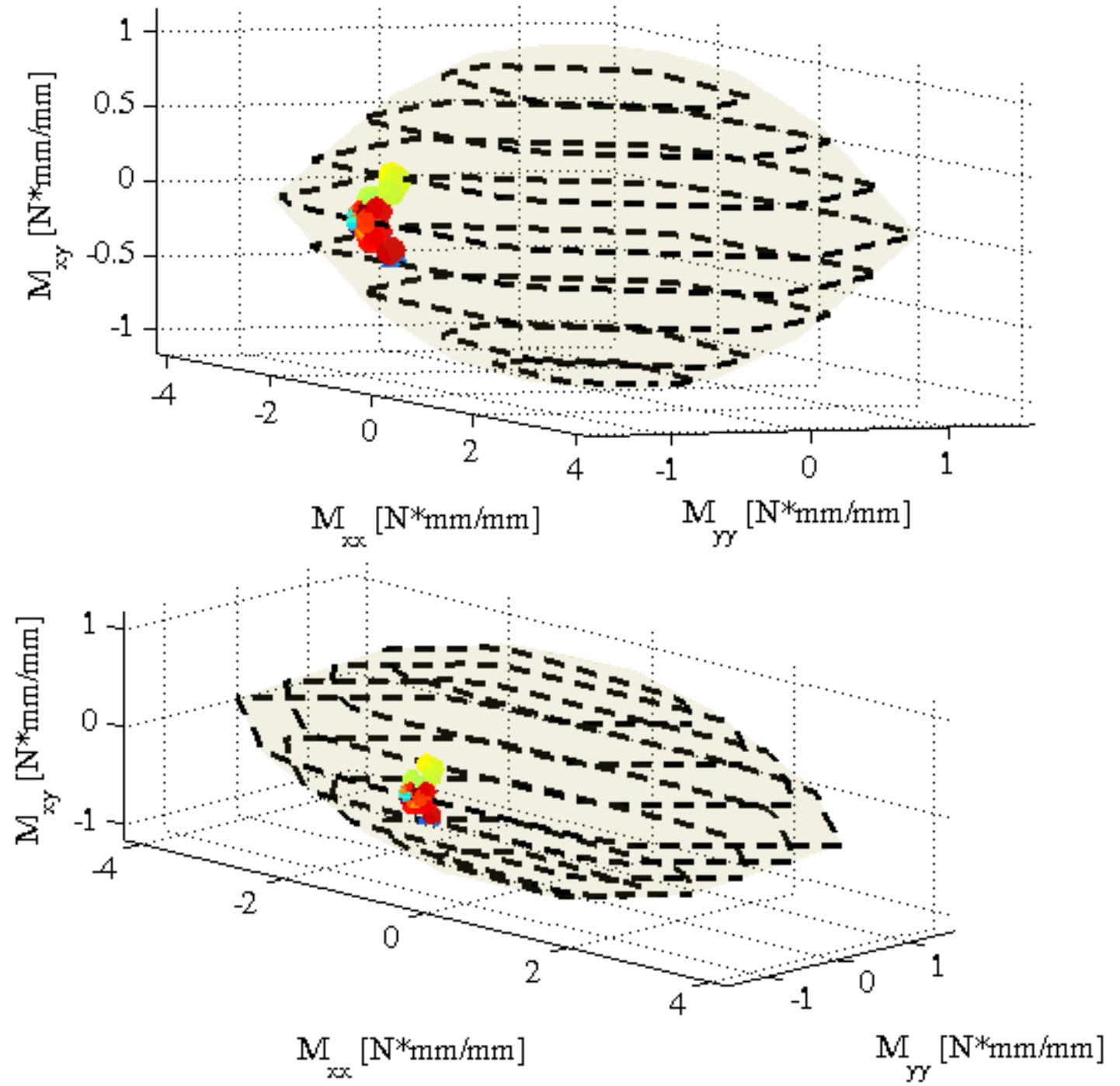

Figure 39: Panel SB03 reinforced through the stress-based optimization for $p=0.155 \mathrm{kN} / \mathrm{m}^{2}$.

Failure surface with 72 planes. -a: Location of the elements where out-of-plane internal actions on masonry layer (bending moment and torque) stand on the failure surface (critical points). -b:

Representation of the failure surface and the critical points on it. 

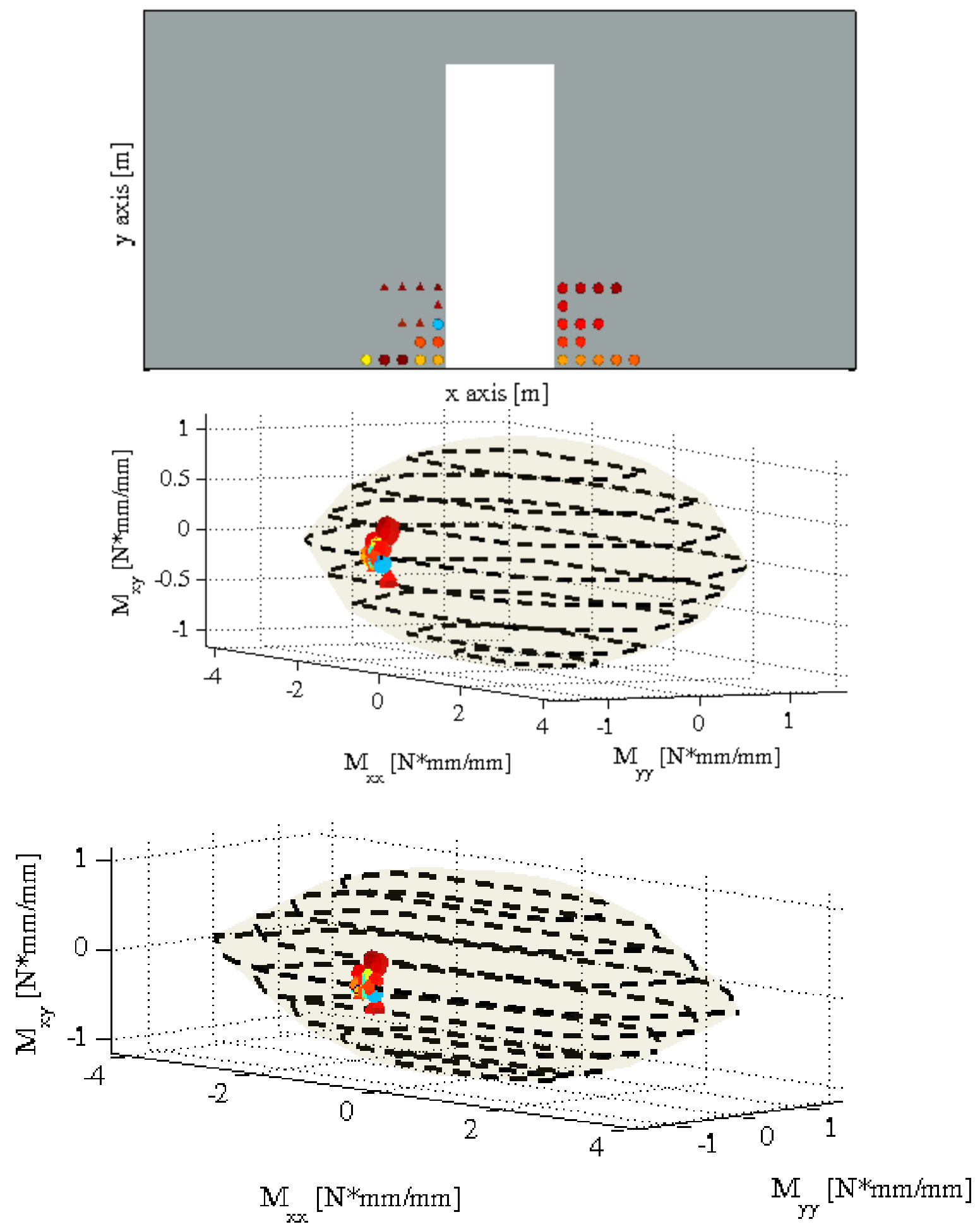

Figure 40: Panel SB03 reinforced through the stress-based optimization for $p=0.155 \mathrm{kN} / \mathrm{m}^{2}$.

Failure surface with 144 planes. -a: Location of the elements where out-of-plane internal actions on masonry layer (bending moment and torque) stand on the failure surface (critical points). -b:

Representation of the failure surface and the critical points on it. 


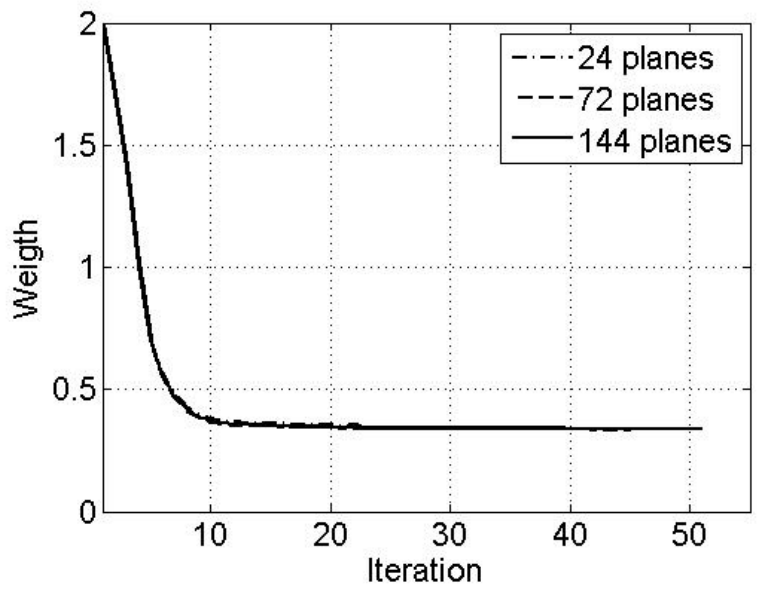

(a)

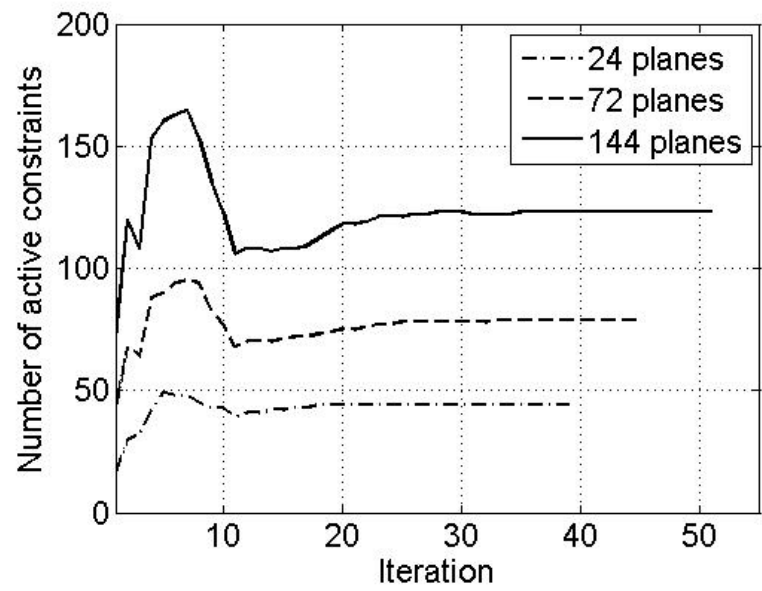

(b)

Figure 41. Reinforcement of Panel SB03 through the stress-based optimization: weight convergence

(a) and number of active constraints (b) for $p=0.155 \mathrm{kN} / \mathrm{m}^{2}$ and three different step-wise approximations of the strength criterion. 


\section{Tables}

\begin{tabular}{|c|c|c|}
\hline & $\begin{array}{c}E\left[\mathrm{~N} / \mathrm{mm}^{2}\right] \\
\text { Young modulus }\end{array}$ & $\begin{array}{c}v \\
\text { Poisson ratio }\end{array}$ \\
\hline brick & 20000 & 0.2 \\
\hline mortar & 5000 & 0.25 \\
\hline
\end{tabular}

Table I: Initial mechanical properties assumed for the elastic simulations reported in Figure 2.

\begin{tabular}{|c|c|c|c|c|}
\hline Symbol & Joint & Brick & & Young Modulus \\
\hline$E$ & 3500 & 10000 & {$[\mathrm{MPa}]$} & Shear Modulus \\
\hline$G$ & 1500 & 5000 & {$[\mathrm{MPa}]$} & Cohesion \\
\hline$c$ & $1.0 f_{t}$ & 2 & {$[\mathrm{MPa}]$} & Tensile strength \\
\hline$f_{t}$ & 0.32 & - & {$[\mathrm{MPa}]$} & Compressive strength \\
\hline$f_{c}$ & 2 & - & {$[\mathrm{MPa}]$} & Friction angle \\
\hline$\Phi$ & 36 & 45 & {$\left[^{\circ}\right]$} & Angle of the linearized compressive cap \\
\hline$\Psi$ & 50 & - & {$\left[^{\circ}\right]$} & $\left.{ }^{\circ}\right]$ \\
\hline
\end{tabular}

Table II: [36][37] experimental data. Mechanical properties assumed for the constituent materials.

\begin{tabular}{|l|c|c|}
\hline \multicolumn{3}{|c|}{$\begin{array}{c}\text { Mortar joints reduced to interfaces } \\
\text { (Mohr-Coulomb failure criterion with tension cutoff and linearized compressive cap) }\end{array}$} \\
\hline cohesion [MPa] & $c$ & $f_{t}$ \\
\hline tensile strength [MPa] & $f_{t}$ & 0.05 \\
\hline compressive strength [MPa] & $f_{c}$ & 2.3 \\
\hline friction angle [Deg] & $\Phi$ & 37 \\
\hline shape of the linearized compressive cap [Deg] & $\Psi$ & 45 \\
\hline \multicolumn{2}{|c|}{ Brick interfaces and triangular brick elements } \\
(Mohr-Coulomb failure criterion)
\end{tabular}

Table III: Mechanical properties adopted for the out-of-plane numerical simulations in presence of vertical pre-compression (standard Italian bricks). 